\title{
Click-on-and-Play
}

Human Motion Capture using Wearable Sensors
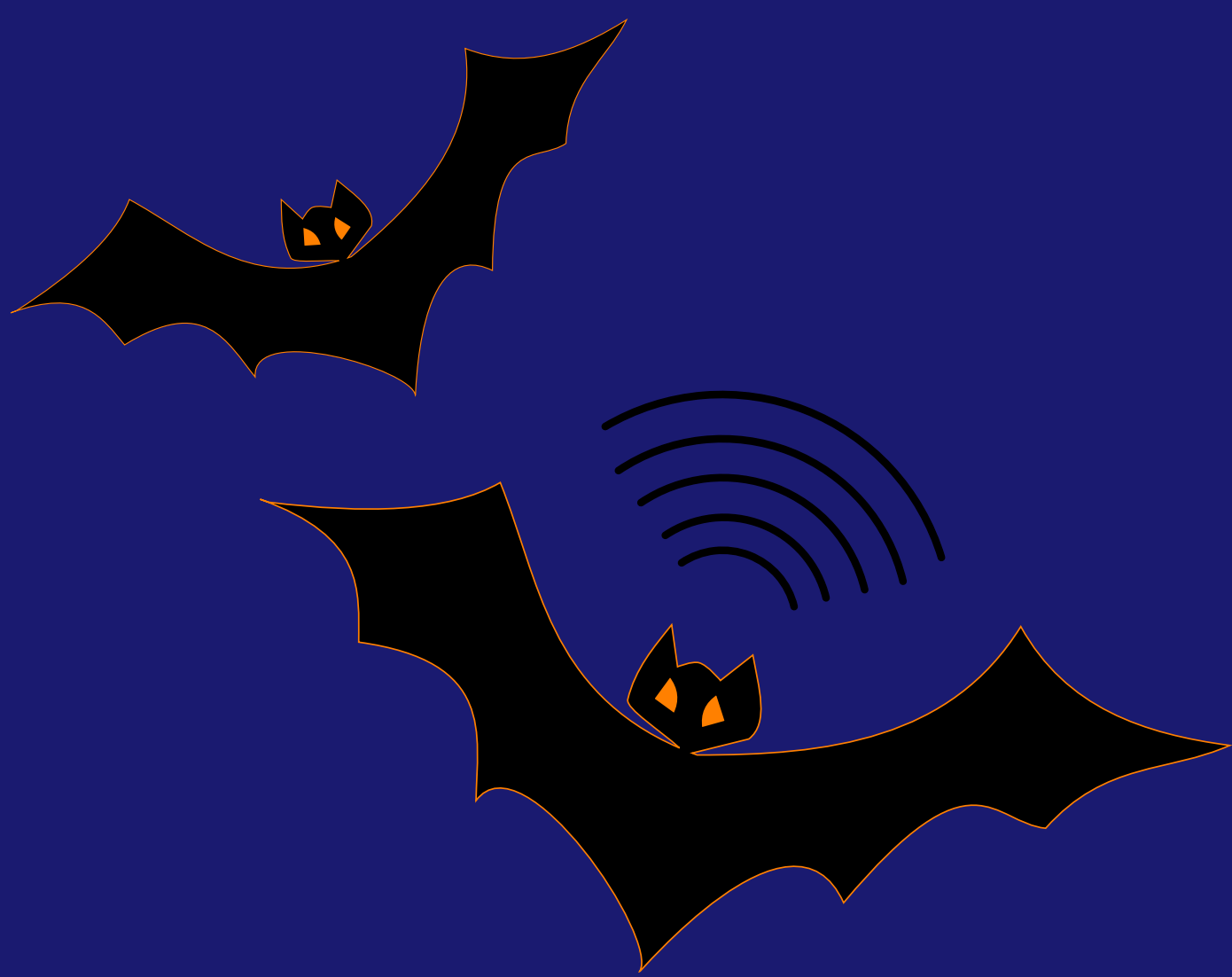

Dirk Weenk 



\section{Click-ON-AND-Play \\ Human Motion Capture using Wearable Sensors}

Dirk Weenk 


\section{Composition of the graduation committee:}

Chairman \& secretary:

Prof. dr. P. M. G. Apers

\section{Supervisors:}

Prof. dr. ir. P. H. Veltink

Prof. dr. ir. H. J. Hermens

\section{Co-supervisor:}

Dr. ir. B. J. F. van Beijnum

\section{Members:}

Prof. dr. ir. C. H. Slump

Prof. dr. J. S. Rietman

Prof. dr. ir. H. F. J. M. Koopman

Univ.-prof. dr. W. Zijlstra

Prof. K. Aminian
University of Twente

University of Twente

University of Twente,

Roessingh Research and Development

University of Twente

University of Twente

University of Twente,

Roessingh Research and Development

University of Twente

German Sports University

Ecole Polytechnique Lausanne

The research described in this thesis is part of the FUSION

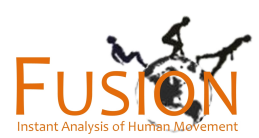
project, funded by PIDON, the Dutch ministry of economic affairs and the provinces of Overijssel and Gelderland and coordinated by ir. C. T. M. Baten, Roessingh Research and Development, Enschede, The Netherlands.

Copyright (c) 2015 by Dirk Weenk, Enschede, The Netherlands

All rights reserved. No part of this publication may be reproduced, stored in a retrieval system, or transmitted, in any form or by any means, electronic, mechanical, photocopying, recording, or otherwise, without the prior written consent of the copyright owner.

ISBN: 978-90-365-3972-2

ISSN: 1381-3617 (CTIT Ph.D. thesis Series No. 15-377)

DOI: $10.3990 / 1.9789036539722$ 


\title{
Click-ON-AND-Play Human Motion Capture using Wearable Sensors
}

\author{
Proefschrift
}

\begin{abstract}
ter verkrijging van
de graad van doctor aan de Universiteit Twente, op gezag van de rector magnificus, prof. dr. H. Brinksma, volgens besluit van het College voor Promoties in het openbaar te verdedigen op vrijdag 4 december 2015 om 12.45 uur
\end{abstract}

door

Dirk Weenk

geboren op 29 juni 1984

te Arnhem 
Dit proefschrift is goedgekeurd door:

De promotoren: prof. dr. ir. P. H. Veltink prof. dr. ir. H. J. Hermens

De co-promotor: dr. ir. B. J. F. van Beijnum 


\section{Summary}

Human motion capture is often used in rehabilitation clinics for diagnostics and monitoring the effects of treatment. Traditionally, camera based systems are used. However, with these systems the measurements are restricted to a lab with expensive cameras. Motion capture outside a lab, using inertial sensors, is becoming increasingly popular to obtain insight in daily-life activity patterns.

There are two main disadvantages of inertial sensor systems. Preparing the measurement system is often a complex and time consuming task. Moreover, it is prone to errors, because each sensor has to be attached to a predefined body segment. Another disadvantage is that inertial sensors cannot measure relative segment positions directly. Especially relative foot positions are very important to be estimated. Together with the center of mass, these positions can be used to assess the balance of a subject. From these two main disadvantages, the goal of this thesis was derived: Contribute to the development of a click-onand-play human motion capture system. This should be a system in which the user attaches (clicks) the sensors to the body segments and can start measuring (play) immediately. Therefore, the following sub-goals were defined. The first goal is to develop an algorithm for the automatic identification of the body segments to which inertial sensors are attached. The second goal is to develop a new sensor system, with a minimal number of sensors, for the estimation of relative foot positions and orientations and the assessment of balance during gait.

The first goal is addressed in chapters 2 and 3 . Chapter 2 presents a method for the automatic identification of body segments on which inertial sensors are positioned. This identification is performed on the basis of a walking trial, assuming the use of a known sensor configuration. Using this method it is possible to distinguish left and right segments. Cross correlations of signals from different measurement units were used and the features were ranked. A decision tree was used for classification of the body segments. When using a full-body configuration (17 different sensor locations), $97.5 \%$ of the sensors were correctly classified. Chapter 3 presents a method that identifies the location of a sensor, without making assumptions about the applied sensor configuration or the activity the user is performing. For a full-body configuration $83.3 \%$ of the sensor locations were correctly classified. Subsequently, for each sensor location a model was developed for activity classification, resulting in a maximum 
accuracy of $91.7 \%$.

The second goal is addressed in the chapters 4, 5 and 6. In chapter 4. ultrasound time of flight is used to estimate the distance between the feet. This system was validated using an optical reference and showed an average error in distance estimation of $7.0 \mathrm{~mm}$. In chapter 5, 3D relative foot positions are estimated by fusing ultrasound and inertial sensor data measured on the shoes in an extended Kalman filter. Step lengths and step widths were calculated and compared to an optical reference system. The mean absolute differences between the two systems were 1.7 and $1.2 \mathrm{~cm}$, for step lengths and step widths, respectively. Chapter 6 describes balance and gait analysis in stroke patients using the shoe-based sensing system described in chapter 5. By combining both kinematics and kinetics, balance of the patients is assessed during gait. A margin of stability - which is the minimal distance from the extrapolated center of mass (projected on the ground) to the base of support - was defined. Both the average walking velocity, as well as the stability margins were smaller for more-affected participants.

In this thesis it is shown that a click-on-and-play human motion capture system is feasible. A method is presented for the identification of body segments to which inertial sensors are attached. This will reduce errors and set-up time of wearable sensor systems. Furthermore, a gait analysis system is presented with sensors only on the feet. Not only is this system ambulant and easy to use, it is also shown to be accurate for gait analysis and balance assessment. 


\section{Samenvatting}

Human motion capture (het vastleggen van menselijke bewegingen) wordt vaak gebruikt in revalidatieklinieken voor diagnose en controle van de effecten van behandelingen. Traditioneel worden hiervoor camerasystemen gebruikt, met als nadeel dat de metingen alleen in een laboratorium met dure camera's kunnen worden verricht. Bewegingsanalyse buiten een laboratorium, met behulp van inertiële sensoren, wordt steeds populairder om inzicht te krijgen in bewegingspatronen van mensen gedurende het dagelijks leven.

Er zijn twee belangrijke nadelen aan het gebruik van inertiële sensorsystemen. Het voorbereiden van het meetsysteem is vaak een complexe en tijdrovende taak. Bovendien is het gevoelig voor fouten, omdat elke sensor aan een vooraf bepaald lichaamsdeel moet worden bevestigd. Een ander nadeel is dat inertiële sensoren de relatieve posities van de lichaamsdelen niet direct kunnen meten. Vooral de relatieve voetposities zijn erg belangrijk om te schatten. Samen met het massamiddelpunt kunnen deze posities worden gebruikt om de balans van een patiënt te beoordelen. Uit deze twee nadelen is het doel van dit proefschrift afgeleid: Bijdragen aan de ontwikkeling van een click-on-andplay systeem voor bewegingsanalyse. Dit moet een systeem zijn waarbij de gebruiker de sensoren op de lichaamssegmenten bevestigt (clicks) en vervolgens direct kan beginnen met meten (play). Aan de hand hiervan zijn de volgende sub-doelen gedefinieerd.

Het eerste doel is om een algoritme te ontwikkelen voor de automatische identificatie van de lichaamsdelen waaraan inertiële sensoren zijn bevestigd. Het tweede doel is om een nieuw sensorsysteem te ontwikkelen, dat met een minimaal aantal sensoren, een schatting van de relatieve posities en oriëntaties van de voeten kan maken. Bovendien kan met dit systeem het evenwicht tijdens lopen beoordeelt worden.

Het eerste doel wordt behandeld in de hoofdstukken 2 en 3 . Hoofdstuk 2 presenteert een methode voor de automatische identificatie van de lichaamsdelen waarop inertiële sensoren zijn gepositioneerd. Deze identificatie wordt uitgevoerd op basis van informatie van de sensoren tijdens het lopen en de gebruikte sensor-configuratie wordt bekend verondersteld. Met deze methode is het mogelijk linker en rechter lichaamsdelen van elkaar te onderscheiden. Hiervoor zijn kruiscorrelaties van signalen van verschillende sensorlocaties gebruikt en eigenschappen van deze signalen zijn gerangschikt van groot naar klein. 
Een beslisboom werd gebruikt voor de classificatie van de lichaamsdelen. Bij gebruik van een 'full-body' configuratie (17 verschillende sensorlocaties) werd $97,5 \%$ van de sensoren correct geïdentificeerd. Hoofdstuk 3 presenteert een methode die de locatie van een sensor identificeert, zonder aannames over de toegepaste sensorconfiguratie of de activiteit die de gebruiker uitvoert. Van een 'full-body' configuratie werd $83,3 \%$ van de sensorlocaties correct geclassificeerd. Vervolgens werd voor elke sensor een model ontwikkeld voor activiteitenclassificatie, wat resulteerde in een maximale nauwkeurigheid van $91,7 \%$.

Het tweede doel wordt behandeld in de hoofdstukken 4, 5 en 6. In hoofdstuk 4 wordt de reistijd van ultrageluid gebruikt om de afstand tussen de voeten te schatten. Dit systeem werd gevalideerd met een optisch referentiesysteem en toonde een gemiddelde fout in de afstandsschatting van 7,0 $\mathrm{mm}$. In hoofdstuk 5 worden 3D relatieve voetposities geschat door data van het ultrasone systeem en inertiële sensoren, gemeten op de schoenen, samen te voegen in een extended Kalman filter. Staplengtes en stapbreedtes werden berekend en vergeleken met een optisch referentiesysteem. De gemiddelde absolute verschillen tussen de twee systemen waren 1.7 en $1.2 \mathrm{~cm}$ voor staplengtes en stapbreedtes, respectievelijk. Hoofdstuk 6 beschrijft evenwicht- en gangbeeldanalyse bij patiënten die een beroerte hebben gehad, met behulp van het schoen-gebaseerde meetsysteem beschreven in hoofdstuk 5. Door het combineren van kinematica en kinetica is de balans van patiënten onderzocht tijdens het lopen. Een zekere stabiliteitsmarge - dat is de minimale afstand van het geëxtrapoleerde lichaamszwaartepunt (geprojecteerd op de grond) tot het draagvlak - is gedefinieerd. Zowel de gemiddelde loopsnelheid, evenals de stabiliteitsmarges bleken kleiner voor patiënten die zwaarder getroffen waren.

In dit proefschrift is aangetoond dat een click-on-and-play motion capture systeem voor de mens, haalbaar is. Er is een methode voorgesteld voor de identificatie van lichaamsdelen waaraan inertiële sensoren zijn bevestigd. Dit zal fouten verminderen en de set-up tijd van draagbare sensorsystemen verkleinen. Verder wordt een gangbeeldanalysesysteem gepresenteerd met sensoren alleen op de voeten. Niet alleen is dit systeem ambulant en eenvoudig te gebruiken, ook is aangetoond dat het nauwkeurig genoeg is voor gangbeeldanalyses en geschikt is voor de evaluatie van evenwicht van personen tijdens het lopen. 


\section{Contents}

Summary vii

Samenvatting ix

\begin{tabular}{lll}
\hline & Introduction & 1
\end{tabular}

1.1 Background . . . . . . . . . . . . . . . . . 3

1.1.1 Traditional motion capture systems . . . . . . . . . . 3

1.1.2 Wearable sensor systems . . . . . . . . . . . . . . . 4

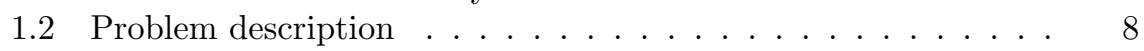

1.3 Research objectives . . . . . . . . . . . . . . 8

1.3.1 A click-on-and-play human motion capture system . . . 9

1.3.2 Thesis goals . . . . . . . . . . . . . . . . . . . . . . . . 9

1.4 Thesis outline . . . . . . . . . . . . . . . . . . 9

2 Automatic identification of inertial sensors during walking $\quad 11$

2.1 Background . . . . . . . . . . . . . . 13

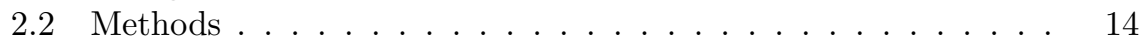

2.2 .1 Measurements . . . . . . . . . . . . . . . . . 14

2.2 .2 Preprocessing . . . . . . . . . . . . . . . . . . . . 15

$2.2 .3 \quad$ Feature extraction . . . . . . . . . . . . . . . 17

2.2.4 Classification for full-body configurations . . . . . . . . 18

2.2.5 Classification for lower body plus trunk configurations . 19

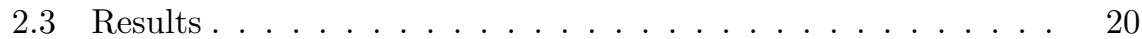

2.3 .1 Full-body configurations . . . . . . . . . . . . . 20

2.3.2 Lower body plus trunk configurations . . . . . . . . . . 22

2.3.3 Testing the algorithms on patients . . . . . . . . . . . 23

2.4 Discussion . . . . . . . . . . . . . . . . . . . . . . . 24

2.5 Conclusions . . . . . . . . . . . . . . . . . . . . . 26

\begin{tabular}{|lll}
3 & On-body inertial sensor location and activity recognition & 29
\end{tabular}

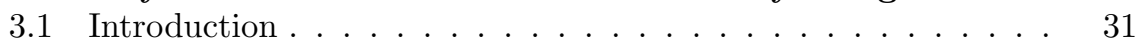

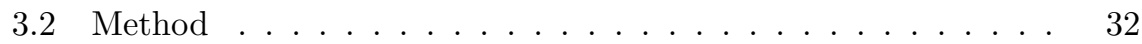

$3.2 .1 \quad$ Experiments . . . . . . . . . . . . . . . . 32

3.2.2 Sensor location and activity recognition method . . . . 33 
3.3 Results. . . . . . . . . . . . . . . . . . . . . 36

3.3 .1 Step 1: Walking recognition . . . . . . . . . . . . . . . . 36

3.3 .2 Step 2: Sensor location recognition . . . . . . . . . . . . 37

3.3 .3 Step 3: Activity recognition . . . . . . . . . . . . . . 37

3.3 .4 Testing the models on stroke patients . . . . . . . . . 37

3.4 Discussion . . . . . . . . . . . . . . . . . . . . . . . 41

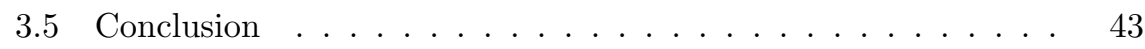

4 Ultrasonic range measurements on the human body 45

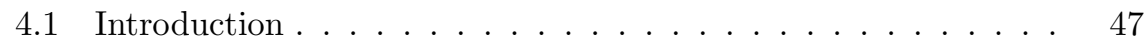

4.2 Design of the sensor $\ldots \ldots \ldots \ldots \ldots$

4.2 .1 Time of flight estimation $\ldots \ldots \ldots \ldots$

4.2 .2 Hardware $\ldots \ldots \ldots \ldots \ldots$

4.3 Validation methods . . . . . . . . . . . . . . . . . . . . 51

4.3 .1 Set-up . . . . . . . . . . . . . . . . . . . . 51

4.3 .2 Reference measurement . . . . . . . . . . . . . . . 51

4.3 .3 Calibration . . . . . . . . . . . . . . . . . . . . . 52

4.3 .4 Synchronization . . . . . . . . . . . . . . . . . 53

4.4 Validation results . . . . . . . . . . . . . . . . . . . . 53

4.4 .1 Calibration measurements . . . . . . . . . . . . . 53

$4.4 .2 \quad$ Walking trials . . . . . . . . . . . . . . . . . . 54

4.5 Discussion . . . . . . . . . . . . . . . . . . . . . . . . . 54

4.6 Conclusion and future work $\ldots \ldots \ldots \ldots$

\begin{tabular}{|lll}
5 & Ambulatory estimation of relative foot positions & 61
\end{tabular}

5.1 Introduction . . . . . . . . . . . . . . . . . . 63

5.2 Sensor fusion method . . . . . . . . . . . . . . . . . . 64

$5.2 .1 \quad$ Sensor signals and models . . . . . . . . . . . . . . . 64

5.2 .2 Filter structure and notations . . . . . . . . . . . . . 67

$5.2 .3 \quad$ Initialization $\ldots \ldots \ldots \ldots \ldots \ldots$

5.2 .4 Prediction . . . . . . . . . . . . . . . . . . . . . . . 68

$5.2 .5 \quad$ Measurement updates $\ldots \ldots \ldots \ldots$. . . . . . . . . 71

$5.2 .6 \quad$ Update orientation and gyro bias . . . . . . . . . . . 73

5.3 Validation method . . . . . . . . . . . . . . . 73

5.3 .1 Set-up . . . . . . . . . . . . . . . . . . . . . . 73

5.3 .2 Synchronization . . . . . . . . . . . . . . . . 74

5.3 .3 Step length and stride width estimation . . . . . . . . 75

5.3 .4 Process noise and measurement noise parameters . . . . 77

5.4 Validation results . . . . . . . . . . . . . . . . . . . 77

5.5 Discussion $\ldots \ldots \ldots \ldots \ldots$

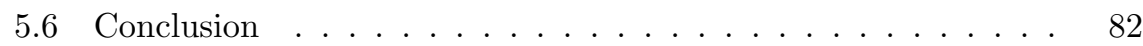


6 Ambulatory assessment of walking balance after stroke 83

6.1 Introduction . . . . . . . . . . . . . 85

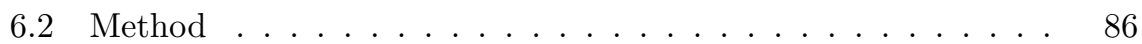

6.2 .1 Participants . . . . . . . . . . . . . . . 86

6.2.2 Experimental protocol . . . . . . . . . . . . . . . 86

6.2 .3 Data processing. . . . . . . . . . . . . . . . . . . 88

6.2 .4 Data analysis . . . . . . . . . . . . . . . . . . . . . 89

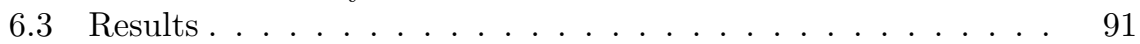

6.4 Discussion . . . . . . . . . . . . . . . . . . . 93

\begin{tabular}{lll}
\hline & Conclusions and discussion & 95
\end{tabular}

7.1 Conclusions . . . . . . . . . . . . . . . 97

7.1.1 Automatic sensor to segment identification . . . . . . . 97

\begin{tabular}{lll}
7.1 .2 & Relative foot position and orientation estimation and bal- \\
\hline & ance assessment. . . . . . . . . . . . . . 97
\end{tabular}

$7.2 \quad$ Discussion and future perspectives . . . . . . . . . . . . . . 98

7.2.1 Automatic sensor to segment identification . . . . . . . 98

\begin{tabular}{|lll}
7.2 .2 & Relative foot position and orientation estimation and bal- \\
\hline & ance assessment . . . . . . . . . . . . . . . 99
\end{tabular}

7.2 .3 Soft-tissue artifacts . . . . . . . . . . . . . . . . . . 100

7.2 .4 Sensor to segment calibration . . . . . . . . . . . . 101

\begin{tabular}{ll}
\hline References & 103
\end{tabular}

\begin{tabular}{ll}
\hline Dankwoord & 115
\end{tabular}

\begin{tabular}{ll}
\hline Biography & 117
\end{tabular}

\begin{tabular}{lr}
\hline List of Publications & 119
\end{tabular} 

Chapter 1

\section{Introduction}




\subsection{Background}

Human motion capture is the process of recording human movements. There are several ways to capture human motion, for example by optical, mechanical, inertial or acoustic sensing. In addition to this kinematic estimation also the kinetic analysis - the estimation of the causes of the movement (i.e. forces and torques) - is important for human movement analysis. Examples of applications of human motion capture are sports training 73 and the animation of movies and games 93 .

The focus of this thesis is on a biomedical application, specifically the use of motion capture in rehabilitation clinics for diagnostics and monitoring the effects of treatment. The quantification of different parameters of the movement is important for this. An example is the study of Lugade et al. [44] in which the center of mass (CoM) and the base of support (BoS) - this is the area under and between the feet - are estimated using reflective markers captured by cameras. The relation between these two measures contains important information about the balance of the subject. Other examples are the studies of Martínez-Ramírez et al. [49, 50, 51] in which patients were monitored before and after total hip arthroplasty during walking and during sit-to-stand transitions. Important information about the individual gait patterns were obtained by measuring movements of patients using instrumented shoes. This information is not represented by gait velocity and questionnaire outcomes that are usually used to assess functional capacity of patients. Another important field of research is activity recognition [2, 81] and coaching, in which the goal is to increase physical activity to prevent diseases [53.

In many applications only parts of the body movements are of interest. For example, the lower extremities are important during gait analysis. Typical outcome measures that need to be quantified in this case are, step or stride lengths and widths, stance and swing times and joint angles [73. However, sometimes the full-body motion needs to be investigated, for example, when studying compensation mechanisms in preventing a fall [36].

\subsubsection{Traditional motion capture systems}

Traditionally, optical systems are used for human motion capture. The positions of multiple markers on the body are measured by cameras positioned in a lab and 3D positions of the body segments are calculated from this information. For measuring ground reaction forces, mostly force plates mounted in the floor are used. An example of a gait lab set-up with cameras and force plates is shown in Figure 1.1. Disadvantages of these lab bound systems are line of sight problems and the fact that only a limited number of steps can be measured inside a lab. Furthermore, movement is restricted because the steps need to be on the force plate 66 . 


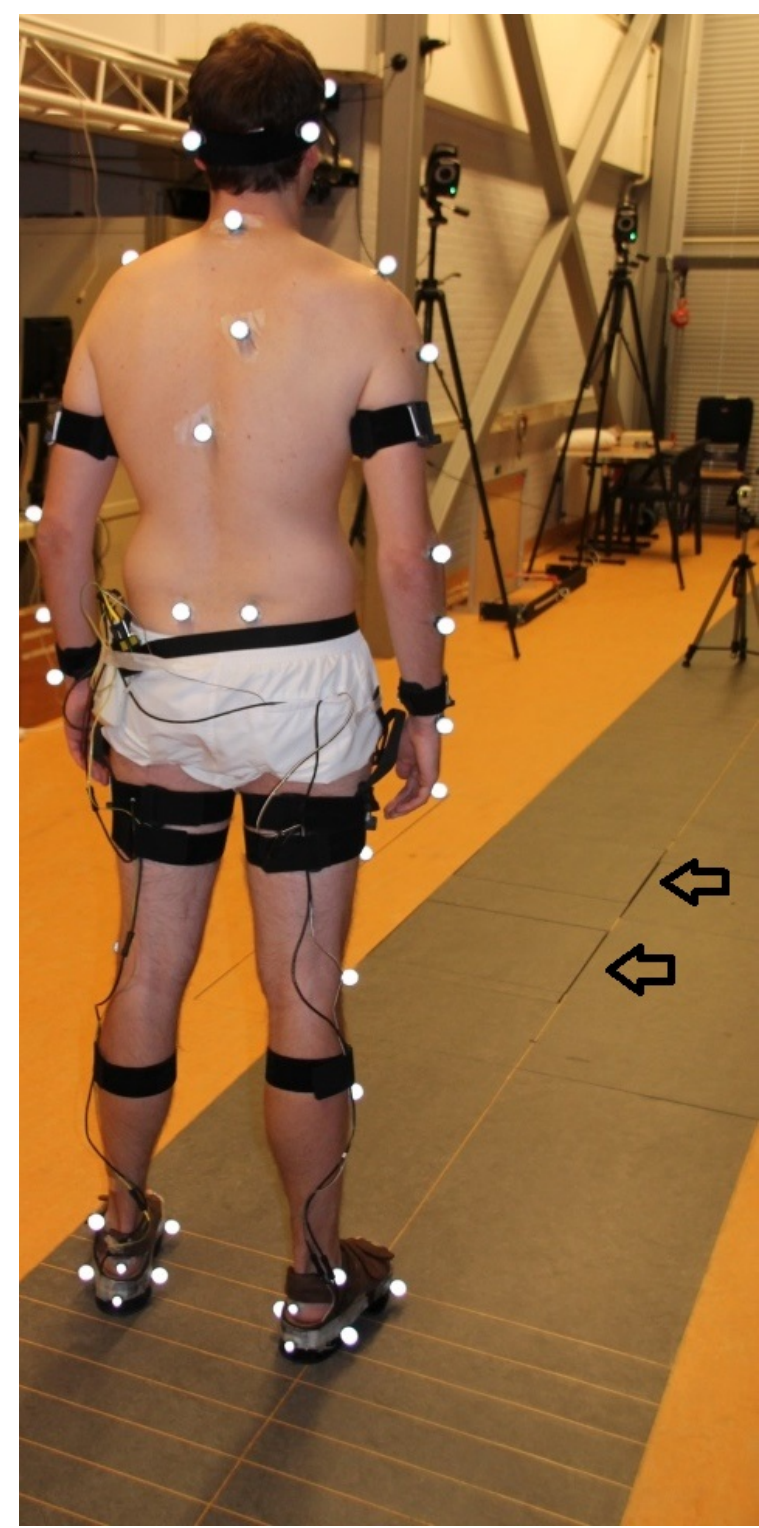

Figure 1.1: Example of a gait-lab set-up. Several cameras are used to capture positions of reflective markers in the body. Force plates in the floor, indicated by the arrows, measure ground reaction forces.

\subsubsection{Wearable sensor systems}

An alternative to traditional lab-bound systems, are wearable sensor systems. With these systems, sensors are attached directly to the body [73]. Advantages 
over traditionally used optical systems include the possibility to perform measurements outside the laboratory and the absence of line of sight problems 62 . Therefore, these systems are becoming increasingly popular. Wearable systems are important for training in sports and performance assessment of patients in an in-home setting [77. An example of a set-up with various wearable sensors is shown in Figure 1.2). Also smartphones that often contain multiple sensors are becoming increasingly popular for monitoring movements of the user [20]. Force and torque sensors in instrumented shoes or pressure insoles are increas-

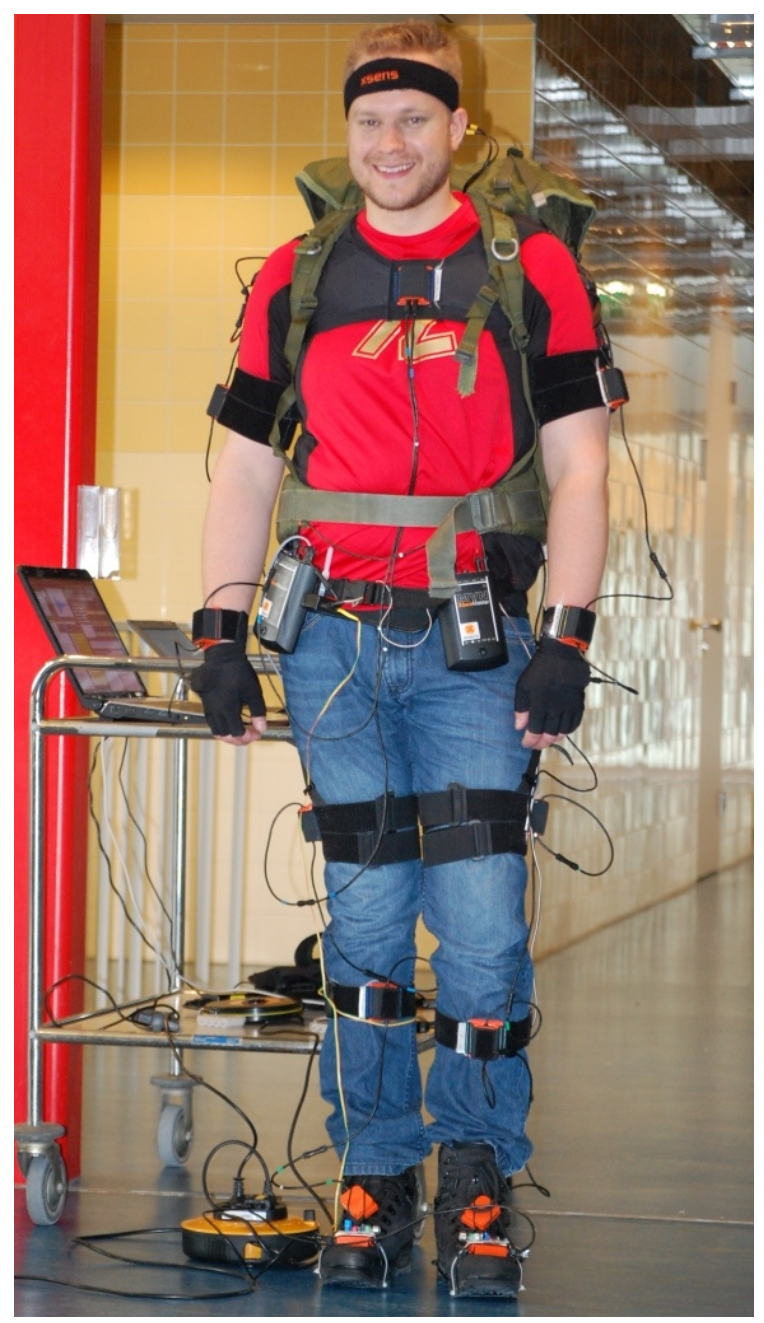

Figure 1.2: Example of various wearable sensors. Xsens full body inertial sensor system together with shoes instrumented with inertial sensors and force sensors in the heel. 
ingly used for kinetic estimation [73. Moments, center of pressure and center of mass can be estimated from the ground reaction forces measured using these sensors 66, 69.

In the remainder of this section, currently available wearable sensor systems are described.

\section{Movement sensors}

Sensors and sensing principles that can be used for movement estimation are for example: flexible goniometers, magnetic sensors, acoustic (time of flight) sensors [80, (wearable) cameras and LEDs 31, barometric pressure sensors 96], laser guidance [21] and radio signal strength [28].

However, the most popular are inertial sensors [46, 58, 66. The principle of inertial sensing is based on measuring forces acting on moving masses [74]. Accelerometers and gyroscopes are both inertial sensors and the combination of both in one device is often referred to as an inertial measurement unit (IMU).

A 3D accelerometer consists of a mass in a box, suspended by springs. The distances between the mass and the box $(\boldsymbol{x})$ are measured (for example using capacitors). Using Hooke's law $(\boldsymbol{F}=k \boldsymbol{x})$, the inertial forces $(\boldsymbol{F})$ acting on the mass $(m)$ are calculated. Next, Newton's second law $(\boldsymbol{F}=m \boldsymbol{a})$ is used to obtain the acceleration $(\boldsymbol{a})$. This acceleration is a combination of the acceleration due to motion and the gravitational acceleration.

Gyroscopes are used to measure 3D angular velocity. If a vibrating mass is rotated with an angular velocity $(\boldsymbol{\omega})$ while it has a translational velocity $(\boldsymbol{v})$, a Coriolis force $\boldsymbol{F}_{C}$ will act on the mass $\left(\boldsymbol{F}_{C}=2 m \boldsymbol{\omega} \times \boldsymbol{v}\right)$. This force causes a vibration orthogonal to the original vibration. From this secondary vibration, the angular velocity is determined.

\section{Force and torque sensors}

Force and torque sensors - when placed under the feet - are important for the estimation of leg loading, joint moments and also for center of mass estimation [73. The instrumented shoe, as shown in Figure 1.3, contains a 6DOF

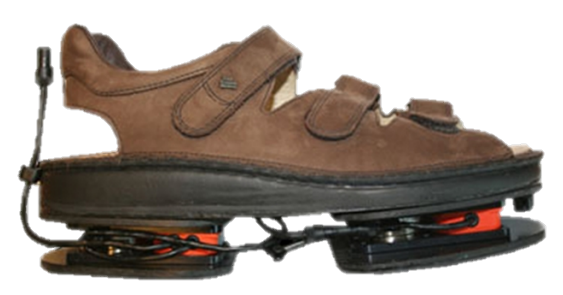

Figure 1.3: Instrumented shoe, containing two $6 \mathrm{D}$ force/moment sensors and two inertial sensors. (ForceShoe ${ }^{\mathrm{TM}}$, Xsens Technologies B.V. 93]) 
force/moment sensor in both the heel and forefoot segment. Also insoles are becoming popular [33, 73]. Although they only measure force in one direction, they are less heavy and easier to include in normal shoes. These wearable force sensor systems allow ambulatory estimation of ground reaction forces, making it suitable for monitoring multiple steps and walking with changes in walking direction. This latter is more difficult with lab-bound systems, since each step has to be on a force plate.

Wearable force and torque sensors in combination with wearable movement sensors have potential for assessing the balance of persons outside a lab environment.

\section{Sensor fusion}

To be able to use accelerometers and gyroscopes for human movement estimation, the information from both sensors needs to be combined (i. e. sensor fusion). The angular velocity of the gyroscopes has to be integrated in order to obtain the (change of) orientation. To obtain the change of position of the IMU, the acceleration from the accelerometer has to be integrated twice. Since the accelerometer measures the sum of the sensor acceleration vector $(\boldsymbol{a})$ and the gravitational acceleration vector $(\boldsymbol{g})$, in sensor coordinate frame, this acceleration has to be transformed to a global (earth fixed) coordinate frame and the gravitational component needs to be removed. To remove the gravitational component, the inclination - that is, the angle of the IMU with respect to the gravity direction - needs to be known over time. Therefore, an accurate orientation estimation is important [83. The double integration of acceleration to obtain position changes, frequently results in integration drift, caused by an offset and noise 68.

Often, the information from all available sensors is combined with a (biomechanical) model consisting of several rigid bodies. These rigid bodies represent the human body segments and are connected by joints. With this model, in combination with a movement measurement system, the positions and orientations of the human body segments and joint angles can be estimated [6, 62, Also the center of mass can be estimated, based on the calculation of the weighted sum of the center of mass position of each segment, using the segment mass as a weighting factor 82 or by combining forces and moments measured under the shoes 69. These calculations mostly take place on a central computer. This computer needs to have knowledge about the segment to which each sensor is attached. One can provide this information by placing each sensor on a predefined body segment. The system also needs to know the orientation of the sensor with respect to segment. This is currently estimated by performing a sensor-segment calibration, in which the user stands in a predefined pose.

Depending on the application, different sensor-configurations can be used. If the interest lies in gait analysis, a lower-body configuration - with sensors on the pelvis, upper legs, lower legs and feet - may suffice. If the application 
is to analyze complete human body movements a full-body configuration is required.

\subsection{Problem description}

A disadvantage of wearable sensing systems is that, in the current situation, the attachment of the sensors is often a complex and time consuming task. As described above, each sensor has to be correctly attached to a predefined body segment and hence this is prone to errors.

Inertial sensors cannot be used to measure relative positions of body segments directly. Especially foot positions are very important to estimate, because their relation with the center of mass is important for the assessment of balance. Also the position of the hand, with respect to the trunk is important for assessing range of motion of a subject. This position information can be obtained from a (biomechanical) model, as was described in the previous section 62. Disadvantage, however, is that this leads to errors when segment lengths or orientations are incorrectly measured or estimated. Moreover, this approach requires many sensor modules on different body segments. Another method for estimating relative positions of body segments, using wearable sensor systems, is with the use of on-body position measurement systems [58, 62, 66, 79]. This is described for example by Roetenberg et al. [59], where the relative positions and orientations of inertial/magnetic sensors on the human body were investigated using a 3D magnetic source, positioned on the back of the body, and 3D magnetic sensors placed at different body segments. The accuracy was approximately $8 \mathrm{~mm}$ in position during movement. Disadvantage is the relatively large size and weight of the magnetic source $(21 \mathrm{~cm}$ diameter, $11 \mathrm{~cm}$ height, $450 \mathrm{~g}$ ), making it unsuitable for placing it on a foot. More recently, Kortier et al. 34 presented a method to estimate the relative position and orientation of a permanent magnet placed on the hand, with respect to four magnetometers placed at the trunk. Although in the presented method a small magnet is used (2 mm radius and $7 \mathrm{~mm}$ length), to cover distances over $70 \mathrm{~cm}$ the magnet needs to be larger (the field strength decreases cubically with distance) or more then four magnetometers, rigidly attached to each other, are needed. Therefore, more research is needed to make the system suitable for relative foot position estimation.

\subsection{Research objectives}

Taking the disadvantages from the previous section into account, several scientific challenges remain, which have been investigated in the FUSION project. These challenges are described in this section followed by the goals of this thesis. 


\subsubsection{A click-on-and-play human motion capture system}

To overcome the disadvantages of the current inertial motion capture systems, the Fusion project was started. The main goal of the FUSION project was 'The development of a Click-on-and-Play Ambulatory 3D Human Motion Capture System' 19. This should be a system in which the user attaches (clicks) the sensors to the body segments and can start measuring (play) immediately. This system should meet several requirements, of which the ones related to this thesis are listed here.

- The system can be used outside a lab and does not restrict the daily-life activities of the user.

- The system can be used by persons without prior knowledge about the system. This means the set-up should be easy, and also the outcome measures of the system should be easy to interpret and give quick and objective insight in the movement that is performed.

- Sensors can be attached to arbitrary body segments, the system recognizes each sensor position and orientation automatically.

- Relative position and orientation of body segments should be estimated accurately. Also an accurate estimation of the center of mass of a subject is required. This will give, together with the relative position of the feet, information about balance of a subject.

\subsubsection{Thesis goals}

The main goal of this thesis is to contribute to the development of a click-onand-play human motion capture system. Based on the requirements mentioned above, the following sub-goals were defined.

1. Develop an algorithm for the automatic identification of the body segments to which IMUs are attached.

2. Develop a new sensor system, with a minimal amount of sensors, for the estimation of relative foot positions and orientations and the assessment of balance during gait. From these estimates, clinically relevant and easy to interpret parameters need to be derived.

\subsection{Thesis outline}

The first goal - to develop an algorithm for the automatic identification of the body segments to which IMUs are attached - is addressed in chapters 2 and 3. In chapter 2 an algorithm for this automatic identification is presented. For this method, data from sensors of a known sensor-configuration are needed and the subject needs to be walking. Chapter 3 presents a method that classifies the sensor locations, without making assumptions about the applied sensor configuration and the activity the user is performing. The second goal - the development of a new sensor systems for the estimation of relative foot positions and orientations and the assessment of balance during gait - is described 
in chapter 4, 5 and 6. In chapter 4 ultrasound time of flight is used to estimate the distance between the feet. In chapter 5, a new fusion algorithm is presented for $3 \mathrm{D}$ relative foot position and orientation estimation using ultrasound and inertial sensor data measured on the shoes. Also in this chapter gait is quantified in terms of step lengths, stride widths, velocity and stance and swing times, making the results easy interpretable outcomes for physicians. In chapter 6 the shoe-based system presented in chapter 5 is used to estimate gait parameters of stroke patients during walking. Also balance is assessed by estimating extrapolated center of mass with respect to base of support. The thesis ends with conclusions and a general discussion in chapter 7. 


\section{Chapter 2}

\section{Automatic identification of iner- tial sensor placement on human body segments during walking}

प

Published as:

D. Weenk, B. J. F. van Beijnum, C. T. M. Baten, H. J. Hermens, P. H. Veltink Automatic identification of inertial sensor placement on human body segments during walking Journal of NeuroEngineering and Rehabilitation 2013 10:31 http://dx.doi.org/10.1186/1743-0003-10-31 


\section{Abstract}

Background Current inertial motion capture systems are rarely used in biomedical applications. The attachment and connection of the sensors with cables is often a complex and time consuming task. Moreover, it is prone to errors, because each sensor has to be attached to a predefined body segment. By using wireless inertial sensors and automatic identification of their positions on the human body, the complexity of the set-up can be reduced and incorrect attachments are avoided.

We present a novel method for the automatic identification of inertial sensors on human body segments during walking. This method allows the user to place (wireless) inertial sensors on arbitrary body segments. Next, the user walks for just a few seconds and the segment to which each sensor is attached is identified automatically.

Methods Walking data was recorded from ten healthy subjects using an Xsens MVN Biomech system with full-body configuration (17 inertial sensors). Subjects were asked to walk for about 6 seconds at normal walking speed (about $5 \mathrm{~km} / \mathrm{h}$ ). After rotating the sensor data to a global coordinate frame with $x$-axis in walking direction, $y$-axis pointing left and $z$-axis vertical, RMS, mean, and correlation coefficient features were extracted from $x$-, $y$ - and $z$-components and magnitudes of the accelerations, angular velocities and angular accelerations. As a classifier, a decision tree based on the C4.5 algorithm was developed using Weka (Waikato Environment for Knowledge Analysis).

Results and conclusions After testing the algorithm with 10-fold crossvalidation using 31 walking trials (involving 527 sensors), 514 sensors were correctly classified (97.5\%). When a decision tree for a lower body plus trunk configuration (8 inertial sensors) was trained and tested using 10-fold crossvalidation, $100 \%$ of the sensors were correctly identified. This decision tree was also tested on walking trials of 7 patients (17 walking trials) after anterior cruciate ligament reconstruction, which also resulted in $100 \%$ correct identification, thus illustrating the robustness of the method. 


\subsection{Background}

$\Upsilon$ ONVENTIONAL human motion capture systems make use of cameras $\checkmark$ and are therefore bounded to a restricted area. This is one of the reasons why over the last few years, inertial sensors (accelerometers and gyroscopes) in combination with magnetic sensors were demonstrated to be a suitable ambulatory alternative. Although accurate 6 degrees of freedom information is available [60, these inertial sensor systems are rarely used in biomedical applications, for example rehabilitation and sports training. This unpopularity could be related to the set-up of the systems. The attachment and connection of the sensors with cables is often a complex and time consuming task. Moreover, it is prone to errors, because each sensor has to be attached to a predefined body segment. Despite the fact that the set-up time for inertial systems is significantly lower $(\leq 15$ minutes for an Xsens MVN Biomech system [93]) than for optical systems [10, it is still a significant amount of time.

However, with decreasing sensor sizes and upcoming wireless inertial sensor technology, the inertial sensors can be attached to the body more easily and quickly, for example using Velcro ${ }^{\circledR}$ straps 98. or even plasters [4]. If it were not necessary to attach each sensor to a predefined segment and if the wired inertial sensors were to be replaced by wireless sensors, the system could be easier to use and both the set-up time and the number of attachment errors could be reduced.

A number of studies on localization of body worn sensors have been conducted previously. Kunze et al. [37, 38, used accelerometer data from 5 inertial sensors combined with various classification algorithms for on-body device localization, resulting in an accuracy of up to $100 \%$ for walking and up to $82 \%$ for arbitrary activities (92\% when using 4 sensors). Amini et al. 1] used accelerometer data of 10 sensors combined with an SVM (support vector machine) classifier to determine the on-body sensor locations. An accuracy of $89 \%$ was achieved. Despite their promising results, several important questions remain. For example, the robustness of these algorithms was not tested on patients with movement disorders. Additionally, a limited number of sensors was used and no method for identifying left and right limbs was presented.

In order for ambulatory movement analysis systems to become generally accepted in biomedical applications, it is essential that the systems become easier to use. By making the systems plug and play, they can be used without having prior knowledge about technical details of the system and they become robust against incorrect sensor placement. This way clinicians or even the patients themselves can attach the sensors, even if they are at home.

In this chapter, a method for automatic identification of body segments to which (wireless) inertial sensors are attached is presented. This method allows the user to place inertial sensors on arbitrary segments of the human body, in a full body- or a lower body plus trunk configuration (17 or 8 inertial sensors respectively). Next, the user walks for just a few seconds and the body segment to which each sensor is attached is identified automatically, based on 
acceleration and angular velocity data. Walking data was used, because it is often used for motion analysis during rehabilitation. In addition to healthy subjects, the method is tested on a group of 7 patients after anterior cruciate ligament (ACL) reconstruction, using a lower body plus trunk configuration.

\subsection{Methods}

\subsubsection{Measurements}

From 11 healthy subjects ( 2 female and 9 male students, all between 20-30 years old), 35 walking trials were recorded using an Xsens MVN Biomech system (Xsens Technologies B.V. [93]) with full body configuration, that is, 17 inertial sensors were placed on 17 different body segments: pelvis, sternum, head, right shoulder, right upper arm, right forearm, right hand, left shoulder, left upper arm, left forearm, left hand, right upper leg, right lower leg, right foot, left upper leg, left lower leg and left foot [92. The subjects, wearing their own daily shoes (no high heels), were asked to stand still for a few seconds and then to start walking at normal speed (about $5 \mathrm{~km} / \mathrm{h}$ ). Because the data was obtained from different previous studies, the number of trials per subject varied from one to four trials. Also the length of the trials varied. From each trial the first 3 walking cycles (about 6 seconds) were used, which was the minimum available number for several trials. Walking cycles were obtained using peak detection of the summation of magnitudes of accelerations and angular velocities of all sensors $\left(\sum_{i=1}^{n}\left(\left\|\boldsymbol{a}_{i}\right\|+\left\|\boldsymbol{\omega}_{i}\right\|\right)\right.$, where $n$ is the number of sensors). One subject (4 trials) showed little to no arm movement during walking and was excluded from the analysis, hence 31 walking trials were used for developing our identification algorithm.

Inertial sensor data - that is, 3D measured acceleration $\left(\boldsymbol{s}^{s}\right)$ and 3D angular velocity $\left(\boldsymbol{\omega}^{s}\right)$, both expressed in sensor coordinate frame - recorded with a sampling frequency of $120 \mathrm{~Hz}$ was saved in MVN file format, converted to XML and loaded into MATLAB ${ }^{\circledR}$ for further analysis.

Besides the full-body configuration a subset of this configuration was analyzed. This lower body plus trunk configuration contained 8 inertial sensors placed on 8 different body segments: pelvis, sternum, upper legs, lower legs and feet. In addition to lower body information, the sternum sensor provides important information about the movement of the trunk. This can be useful in applications where balance needs to be assessed.

In order to test the robustness of the algorithm, 17 walking trials of 7 patients ( 1 female, 6 male, age $28 \pm 8.35$ ) after anterior cruciate ligament (ACL) reconstruction were used. These trials were recorded using an Xbus Kit (Xsens Technologies B.V. 93]) during a study of Baten et al. [3]. In their study 7 patients were measured four times during the rehabilitation process, with an interval of one month. To test the robustness of our identification algorithm, the first measurements - approximately 5 weeks after the ACL reconstruction, 


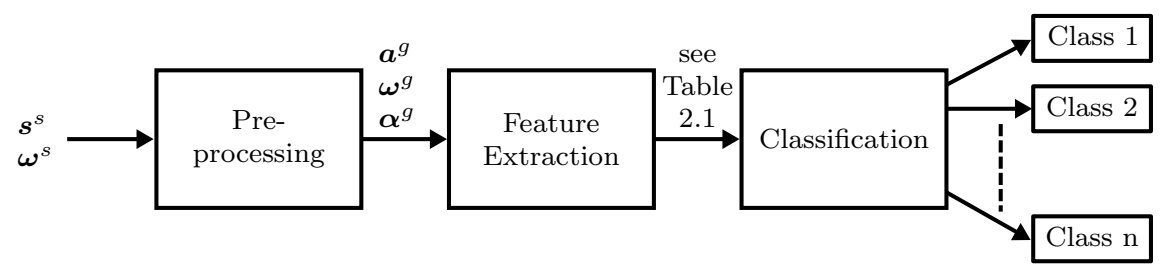

Figure 2.1: The three steps used for identifying the inertial sensors. Inputs are the measured 3D acceleration $\left(\boldsymbol{s}^{s}\right)$ and angular velocity $\left(\boldsymbol{\omega}^{s}\right)$, both expressed in sensor coordinate frame. Outputs of the identification process are the classes, in this case the body segments to which the inertial sensors are attached.

where walking asymmetry was largest - were used. No medical ethical approval was required under Dutch regulations, given the materials and methods used. The research was in full compliance with the "Declaration of Helsinki" and written informed consent was obtained from all patients for publication of the results.

\subsubsection{Preprocessing}

Identification of the inertial sensors was split into three steps: preprocessing, feature extraction and classification (Figure 2.1). To be able to compare the sensors between different body segments and different subjects, the accelerations and angular velocities were pre-processed; that is, the gravitational accelerations were subtracted from the accelerometer outputs and the 3D sensor signals were all transformed to the global coordinate frame $\psi_{g}$ with the $z$-axis pointing up, the $x$-axis in the walking direction and the $y$-axis pointing left.

To transform the 3D accelerations and angular velocities from sensor coordinate frame $\psi_{s}$ to global coordinate frame $\psi_{g}$, the orientation of the inertial sensor - with respect to the global coordinate frame - had to be estimated. For this purpose, first the inclination of the sensors was estimated when the subjects were standing still, by using the accelerometers that measure the gravitational acceleration under this condition. When the subjects were walking, the change of orientation of the sensors was estimated using the gyroscopes by integrating the angular velocities. The following differential equation was solved to integrate the angular velocities to angles [66]:

$$
\dot{\boldsymbol{R}}_{s}^{g^{\prime}}=\boldsymbol{R}_{s}^{g^{\prime}} \tilde{\boldsymbol{\omega}}^{s}
$$

where the $3 \mathrm{D}$ rotation matrix $\boldsymbol{R}_{s}^{g^{\prime}}$ represents the change of coordinates from $\psi_{s}$ to a frame $\psi_{g^{\prime}}$ with all vertical axes aligned, but with the heading in the original (unchanged) direction. $\tilde{\boldsymbol{\omega}}^{s}$ is a skew-symmetric matrix consisting of the components of the angular velocity vector expressed in $\psi_{s}$ :

$$
\tilde{\boldsymbol{\omega}}^{s}=\left(\begin{array}{ccc}
0 & -\omega_{z} & \omega_{y} \\
\omega_{z} & 0 & -\omega_{x} \\
-\omega_{y} & \omega_{x} & 0
\end{array}\right)
$$


where the indices ()$^{s}$ are omitted for readability (see also [66]). For the 3D sensor acceleration in frame $\psi_{g^{\prime}}$, denoted $\boldsymbol{a}^{g^{\prime}}(t)$, the following equation holds:

$$
\boldsymbol{a}^{g^{\prime}}(t)=\boldsymbol{R}_{s}^{g^{\prime}}(t) \boldsymbol{s}^{s}(t)+\boldsymbol{g}^{g^{\prime}}
$$

where $\boldsymbol{s}^{s}(t)$ is the measured acceleration and $\boldsymbol{g}^{g^{\prime}}$ is the gravitational acceleration expressed in $\psi_{g^{\prime}}$ (assumed to be constant and known), which was subsequently subtracted from the $z$-component of the 3D sensor acceleration. The rotation matrix $\boldsymbol{R}_{s}^{g^{\prime}}(t)$ was also used to express $\boldsymbol{\omega}^{s}$ in $\psi_{g^{\prime}}$ :

$$
\boldsymbol{\omega}^{g^{\prime}}(t)=\boldsymbol{R}_{s}^{g^{\prime}}(t) \boldsymbol{\omega}^{s}(t)
$$

After aligning the vertical axes, the heading was aligned by aligning the positive $x^{g^{\prime}}$-axis with the walking direction, which was obtained by integrating the acceleration in frame $\psi_{g^{\prime}}$ - yielding the velocity $\boldsymbol{v}^{g^{\prime}}-$ using trapezoidal numerical integration. From $\boldsymbol{v}^{g^{\prime}}$, the $x$ and $y$ components were used to obtain the angle (in the horizontal plane) with the positive $x$-axis $\left(\boldsymbol{x}^{g^{\prime}}\right)$. Drawback of this method is the drift caused by integrating noise and sensor bias. The effect of this integration drift on the estimation of the walking direction was reduced by using the mean of the velocity of the first full walking cycle to estimate the walking direction, assuming that this gave a good estimate of the walking direction of the complete walking trial.

The angle $\theta$ (in the horizontal plane) between $\boldsymbol{x}^{g^{\prime}}$ and the velocity vector $\boldsymbol{v}^{g^{\prime}}$ was obtained using:

$$
\theta=\arccos \left(\frac{\boldsymbol{x}^{g^{\prime}} \cdot \boldsymbol{v}^{g^{\prime}}}{\left\|\boldsymbol{x}^{g^{\prime}}\right\|\left\|\boldsymbol{v}^{g^{\prime}}\right\|}\right)
$$

This angle was then used to obtain the rotation matrix:

$$
\boldsymbol{R}_{g^{\prime}}^{g}(\theta)=\left(\begin{array}{ccc}
\cos \theta & -\sin \theta & 0 \\
\sin \theta & \cos \theta & 0 \\
0 & 0 & 1
\end{array}\right)
$$

which was used (as in 2.4 ) to rotate the accelerations $\left(\boldsymbol{a}^{g^{\prime}}\right)$ and angular velocities $\left(\boldsymbol{\omega}^{g^{\prime}}\right)$ of all the sensors to global coordinate frame $\psi_{g}$, with $x$-axis in walking direction, $y$-axis pointing left and $z$-axis vertical.

To obtain additional information about (rotational) accelerations, which are invariant to the position on the segment, the 3D angular acceleration $\boldsymbol{\alpha}^{g}$ was calculated:

$$
\boldsymbol{\alpha}^{g}=\frac{d \boldsymbol{\omega}^{g}}{d t}
$$

In the remainder of this chapter $\boldsymbol{a}, \boldsymbol{\omega}$ and $\boldsymbol{\alpha}$ are always expressed in frame $\psi_{g}$, the index ()$^{g}$ is omitted for readability. 
Table 2.1: Features used for identifying the inertial sensors. All $57(19 \times 3)$ features are given as input to the decision tree learner. The C4.5 algorithm automatically chooses the features that split the data most effectively.

\begin{tabular}{|c|c|c|c|}
\hline \multirow[b]{2}{*}{ Description } & \multicolumn{3}{|c|}{ Feature } \\
\hline & $a$ & $\omega$ & $\alpha$ \\
\hline $\begin{array}{l}\text { RMS of the } \\
\text {-magnitude } \\
-x \text {-component } \\
-y \text {-component } \\
-z \text {-component }\end{array}$ & $\begin{array}{l}\operatorname{RMS}\{\|\boldsymbol{a}\|\} \\
\operatorname{RMS}\left\{a_{x}\right\} \\
\operatorname{RMS}\left\{a_{y}\right\} \\
\operatorname{RMS}\left\{a_{z}\right\}\end{array}$ & $\begin{array}{c}\operatorname{RMS}\{\|\boldsymbol{\omega}\|\} \\
\operatorname{RMS}\left\{\omega_{x}\right\} \\
\operatorname{RMS}\left\{\omega_{y}\right\} \\
\operatorname{RMS}\left\{\omega_{z}\right\}\end{array}$ & $\begin{array}{c}\operatorname{RMS}\{\|\boldsymbol{\alpha}\|\} \\
\operatorname{RMS}\left\{\alpha_{x}\right\} \\
\operatorname{RMS}\left\{\alpha_{y}\right\} \\
\operatorname{RMS}\left\{\alpha_{z}\right\}\end{array}$ \\
\hline $\begin{array}{l}\text { Variance of the } \\
\text {-magnitude } \\
\text {-x-component } \\
\text {-y-component } \\
\text { - } z \text {-component }\end{array}$ & $\begin{array}{c}\operatorname{Var}\{\|\boldsymbol{a}\|\} \\
\operatorname{Var}\left\{a_{x}\right\} \\
\operatorname{Var}\left\{a_{y}\right\} \\
\operatorname{Var}\left\{a_{z}\right\}\end{array}$ & $\begin{array}{c}\operatorname{Var}\{\|\boldsymbol{\omega}\|\} \\
\operatorname{Var}\left\{\omega_{x}\right\} \\
\operatorname{Var}\left\{\omega_{y}\right\} \\
\operatorname{Var}\left\{\omega_{z}\right\}\end{array}$ & $\begin{array}{c}\operatorname{Var}\{\|\boldsymbol{\alpha}\|\} \\
\operatorname{Var}\left\{\alpha_{x}\right\} \\
\operatorname{Var}\left\{\alpha_{y}\right\} \\
\operatorname{Var}\left\{\alpha_{z}\right\}\end{array}$ \\
\hline $\begin{array}{l}\text { Sum of cc's of a } \\
\text {-magnitude } \\
\text { - } x \text {-component } \\
\text { - } y \text {-component } \\
\text { - } z \text {-component }\end{array}$ & $\begin{array}{l}\text { sensor with all o } \\
\qquad \operatorname{cc}\{\|\boldsymbol{a}\|\} \\
\Sigma \operatorname{cc}\left\{a_{x}\right\} \\
\Sigma \operatorname{cc}\left\{a_{y}\right\} \\
\Sigma \operatorname{cc}\left\{a_{z}\right\}\end{array}$ & $\begin{array}{l}\text { ner sensors of the } \\
\Sigma \operatorname{cc}\{\|\boldsymbol{\omega}\|\} \\
\Sigma \operatorname{cc}\left\{\omega_{x}\right\} \\
\Sigma \operatorname{cc}\left\{\omega_{y}\right\} \\
\Sigma \operatorname{cc}\left\{\omega_{z}\right\}\end{array}$ & $\begin{array}{c}\Sigma \operatorname{cc}\{\|\boldsymbol{\alpha}\|\} \\
\Sigma \operatorname{cc}\left\{\alpha_{x}\right\} \\
\Sigma \operatorname{cc}\left\{\alpha_{y}\right\} \\
\Sigma \operatorname{cc}\left\{\alpha_{z}\right\}\end{array}$ \\
\hline $\begin{array}{l}\text { The maximum } \\
\text {-magnitude } \\
\text { - } x \text {-component } \\
\text { - } y \text {-component } \\
\text { - } z \text {-component }\end{array}$ & $\begin{array}{c}\text { ralue of the cc's } \\
\operatorname{Max}\{\operatorname{cc}\{\|\boldsymbol{a}\|\}\} \\
\operatorname{Max}\left\{\operatorname{cc}\left\{a_{x}\right\}\right\} \\
\operatorname{Max}\left\{\operatorname{cc}\left\{a_{y}\right\}\right\} \\
\operatorname{Max}\left\{\operatorname{cc}\left\{a_{z}\right\}\right\}\end{array}$ & $\begin{array}{c}\text { a sensor with all } \\
\operatorname{Max}\{\operatorname{cc}\{\|\boldsymbol{\omega}\|\}\} \\
\operatorname{Max}\left\{\operatorname{cc}\left\{\omega_{x}\right\}\right\} \\
\operatorname{Max}\left\{\operatorname{cc}\left\{\omega_{y}\right\}\right\} \\
\operatorname{Max}\left\{\operatorname{cc}\left\{\omega_{z}\right\}\right\}\end{array}$ & $\begin{array}{l}\text { her sensors of the } \\
\operatorname{Max}\{\operatorname{cc}\{\|\boldsymbol{\alpha}\|\}\} \\
\operatorname{Max}\left\{\operatorname{cc}\left\{\alpha_{x}\right\}\right\} \\
\operatorname{Max}\left\{\operatorname{cc}\left\{\alpha_{y}\right\}\right\} \\
\operatorname{Max}\left\{\operatorname{cc}\left\{\alpha_{z}\right\}\right\}\end{array}$ \\
\hline $\begin{array}{l}\text { The inter-axis c } \\
-x \text { - and } y \text {-axes } \\
-x \text { - and } z \text {-axes } \\
-y \text { - and } z \text {-axes }\end{array}$ & $\begin{array}{c}\text { c's of a sensor be } \\
\quad \operatorname{cc}\left\{a_{x}, a_{y}\right\} \\
\operatorname{cc}\left\{a_{x}, a_{z}\right\} \\
\operatorname{cc}\left\{a_{y}, a_{z}\right\}\end{array}$ & $\begin{array}{l}\text { veen the } \\
\qquad \begin{array}{l}\operatorname{cc}\left\{\omega_{x}, \omega_{y}\right\} \\
\operatorname{cc}\left\{\omega_{x}, \omega_{z}\right\} \\
\operatorname{cc}\left\{\omega_{y}, \omega_{z}\right\}\end{array}\end{array}$ & $\begin{array}{l}\operatorname{cc}\left\{\alpha_{x}, \alpha_{y}\right\} \\
\operatorname{cc}\left\{\alpha_{x}, \alpha_{z}\right\} \\
\operatorname{cc}\left\{\alpha_{y}, \alpha_{z}\right\}\end{array}$ \\
\hline
\end{tabular}

\subsubsection{Feature extraction}

Features were extracted from magnitudes as well as from the $x^{-}, y-$, and $z$ components of the 3D accelerations $(\boldsymbol{a})$, angular velocities $(\boldsymbol{\omega})$ and angular accelerations $(\boldsymbol{\alpha})$. The features that were extracted are RMS, variance, correlation coefficients (cc's) between (the same components of) sensors on different segments, and inter-axis correlation coefficients (of single sensors) and are listed in Table 2.1.

Because the correlation coefficients were in matrix form, they could not be inserted directly as features (because the identity of the other sensors was unknown). For this reason, the sum of the correlation coefficients of a sensor with all other sensors and the maximum value of the correlation coefficients of a sensor with the other sensors were used as features. This corresponds to the sums and the maximum values of each row (neglecting the autocorrelations on the diagonal) of the correlation matrix respectively and gives an impression of the correlation of a sensor with all other sensors. Minimal values and the sum of the absolute values of the correlation coefficients were also investigated, but 
did not contribute to the identification of the sensors.

\subsubsection{Classification for full-body configurations}

Following feature extraction, Weka (Waikato Environment for Knowledge Analysis), a collection of machine learning algorithms for data mining tasks [25, 89], was used for the classification of the inertial sensors.

In this study decision trees were used for classification, because they are simple to understand and interpret, they require little data preparation, and they perform well with large datasets in a short time [2, 91.

The datasets for classification contained instances of 31 walking trials of 17 sensors each. All 57 features that are listed in Table 2.1 were given as input to Weka. The features were ranked, using fractional ranking (also known as "1 2.52 .5 4" ranking: equal numbers receive the mean of what they would receive when using ordinal ranking), to create ordinal features. This was done to minimize variability between individuals and between different walking speeds. This ranking process of categorizing the features is a form of classification and can only be used when the sensor-configuration is known beforehand (in this case it was known that a full-body configuration was used). A drawback of this ranking process is that the distance between the feature values (and thus the physical meaning) is removed.

In Weka, the J4.8 decision tree classifier - which is an implementation of the C4.5 algorithm - with default parameters was chosen. As a test option, a 10-fold cross-validation was chosen because in the literature this has been shown to be a good estimate of the error rate for many problems 91.

The C4.5 algorithm builds decision trees from a set of training data using the concept of information entropy. Information entropy $H$ (in bits) is a measure of uncertainty and is defined as:

$$
H=-\sum_{i=1}^{n} p(i) \log _{2}(p(i))
$$

where $n$ is the number of classes (in this case body segments) and $p(i)$ is the probability that a sensor is assigned to class $i$. This probability is defined as the number of sensors attached to segment $i$ divided by the total number of sensors. Information gain is the difference in entropy, before and after selecting one of the features to make a split [15, 91.

At each node of the decision tree, the $\mathrm{C} 4.5$ algorithm chooses one feature of the dataset that splits the data most effectively, that is, the feature with the highest information gain is chosen to make the split.

The main steps of the C4.5 algorithm are [15, 91]:

1. If all (remaining) instances (sensors) belong to the same class (segment), then finish

2. Calculate the information gain for all features

3. Use the feature with the largest information gain to split the data

4. Repeat steps 1 to 3 . 
To improve robustness, the classification was split into three steps. In the first step the body segments were classified without looking at left or right (or contra-/ipsilateral), while in the next steps the distinction between left and right was made.

\section{Step one - segment identification}

In the first step, the body segments were identified, without distinguishing left and right. The features were ranked 1-17, but sensors were classified in ten different classes (pelvis, sternum, head, shoulder, upper arm, forearm, hand, upper leg, lower leg and foot), using Weka as described above.

\section{Step two - left and right upper arm and upper leg identification}

When segments were identified in step 1, left and right upper legs (and arms) were identified using correlation coefficients between pelvis-sensor (sternumsensor for the upper arms) orientation $\theta$ and upper leg (or arm) movement.

The sternum- and pelvis-sensor orientation $\theta$ about $x, y$ and $z$ axes were obtained by trapezoidal numerical integration of angular velocity, followed by detrending. In this case it was not necessary to use differential equation (2.1), because in all directions only small changes in orientation were measured on these segments. This provides left and right information, because of the coordinate frame transformation described before in the preprocessing Section (the $y$-axis points left). For the upper arms and upper legs, accelerations, velocities, angular velocities, angular accelerations and orientations of $x, y$ and $z$ axes were used.

Correlation coefficients of 45 combinations of $x, y, z$ components were calculated, ranked and used to train a decision tree using the same method as described above.

Step three - left and right identification for shoulders, forearms, hands, lower legs and feet

Left and right identification of the remaining segments (shoulders, forearms, hands, lower legs and feet) was done using correlation coefficients between $(x$, $y, z$ or magnitude) accelerations and angular velocities of sensors on adjacent segments for which it is known whether they are left or right.

\subsubsection{Classification for lower body plus trunk configura- tions}

The classification for a lower body plus trunk configuration was similar to the full-body configuration, but instead of 17 inertial sensors, only 8 inertial sensors (on pelvis, sternum, right upper leg, left upper leg, right lower legs, left lower leg, right foot and left foot) were used. In the first step the features were now ranked 1-8, but sensors were classified in 5 different classes (pelvis, sternum, 
upper leg, lower leg, foot). In steps 2 and 3 the distinction between left and right was made again. The decision trees were trained using the 31 trials of the healthy subjects and subsequently tested, using 10-fold cross-validation, on these 31 trials and also on 17 trials of 7 patients after ACL reconstruction.

\subsection{Results}

\subsubsection{Full-body configurations}

The results of the three steps are described individually below.

\section{Step one - segment identification}

The J4.8 decision tree classifier, as constructed using Weka, is shown in Figure 2.2. The corresponding confusion matrix is shown in Table 2.2. From the $(31 \cdot 17=) 527$ inertial sensors, 514 were correctly classified $(97.5 \%)$.

The decision making is based on the ranking of the features. For example, when looking at the top of the decision tree (at the first split) the 6 sensors (of each trial) with the largest RMS magnitude of the acceleration (RMS $\{\|\boldsymbol{a}\|\}$ ) are separated from the rest. These are the upper legs, lower legs and feet. Consequently the other 11 sensors of each walking trial are the pelvis, sternum, head, shoulders, upper arms, forearms and hand.

\section{Step two - left and right upper arm and upper leg identification}

In Figure 2.3 the decision trees that were constructed for left and right upper arm and upper leg identification are shown. The left Figure indicates that, to identify left and right upper arms, from both upper arm sensors the correlation of the acceleration in $z$ direction with the sternum sensor orientation about the $x$-axis has to be calculated. The sensor which results in the largest correlation

Table 2.2: Confusion matrix resulting from testing the decision tree in Figure 2.2 with 10 -fold cross-validation, using 31 walking trials. From the $(31 \cdot 17=) 527$ inertial sensors, 514 were correctly classified (97.5\%).

\begin{tabular}{rrrrrrrrrll}
\hline $\mathrm{a}$ & $\mathrm{b}$ & $\mathrm{c}$ & $\mathrm{d}$ & $\mathrm{e}$ & $\mathrm{f}$ & $\mathrm{g}$ & $\mathrm{h}$ & $\mathrm{i}$ & $\mathrm{j}$ & $<$ - classified as \\
\hline 30 & 0 & 1 & 0 & 0 & 0 & 0 & 0 & 0 & 0 & $\mathrm{a}=$ Pelvis \\
0 & 25 & 0 & 6 & 0 & 0 & 0 & 0 & 0 & 0 & $\mathrm{~b}=$ Sternum \\
1 & 0 & 30 & 0 & 0 & 0 & 0 & 0 & 0 & 0 & $\mathrm{c}=$ Head \\
0 & 1 & 0 & 61 & 1 & 0 & 0 & 0 & 0 & 0 & $\mathrm{~d}=$ Shoulder \\
0 & 0 & 0 & 0 & 61 & 0 & 0 & 0 & 0 & 0 & $\mathrm{e}=$ Upper arm \\
0 & 0 & 0 & 0 & 0 & 62 & 0 & 0 & 0 & 0 & $\mathrm{f}=$ Forearm \\
0 & 0 & 0 & 0 & 0 & 3 & 59 & 0 & 0 & 0 & $\mathrm{~g}=$ Hand \\
0 & 0 & 0 & 0 & 0 & 0 & 0 & 62 & 0 & 0 & $\mathrm{~h}=$ Upper leg \\
0 & 0 & 0 & 0 & 0 & 0 & 0 & 0 & 62 & 0 & $\mathrm{i}=$ Lower leg \\
0 & 0 & 0 & 0 & 0 & 0 & 0 & 0 & 0 & 62 & $\mathrm{j}=$ Foot \\
\hline
\end{tabular}




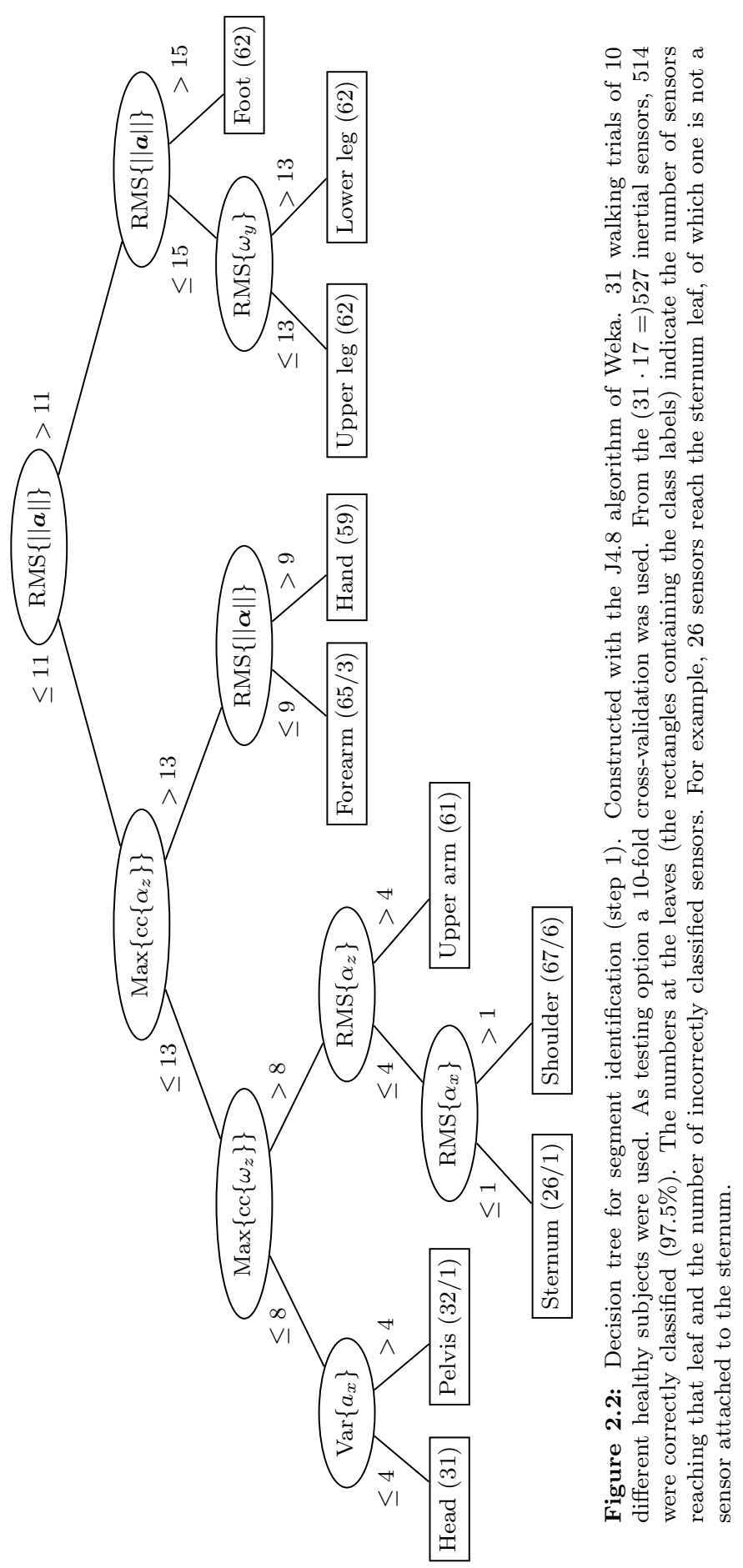




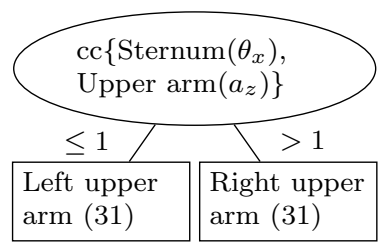

(a) Left/right upper arm

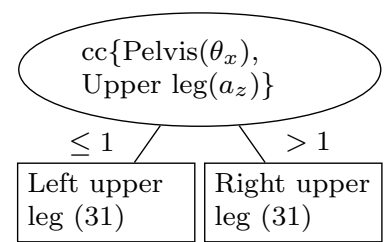

(b) Left/right upper leg

Figure 2.3: Decision trees for left and right upper arm and upper leg identification in step 2. To identify left and right upper arms, from both upper arm sensors the correlation of the acceleration in $z$ direction with the sternum sensor orientation about the $x$-axis was used (left). For the upper legs the orientation of the pelvis sensor was used (right). For these segments, all sensors were identified correctly (100\% accuracy)

coefficient is the sensor on the right upper arm. For the upper legs the orientation of the pelvis sensor is used instead of the sternum sensor (right Figure). For these segments, all sensors were identified correctly (100\% accuracy).

\section{Step three - left and right identification for shoulders, forearms, hands, lower legs and feet}

Table 2.3 lists the correlation coefficients for left and right identification of the remaining segments (shoulders, forearms, hands, lower legs and feet), determined using Weka. For example, to identify left and right shoulders, the correlation coefficients of acceleration in $z$-direction between shoulders and upper arms (from which left and right were determined in the previous step) have to be calculated. The largest correlation coefficient then indicates whether segments are on the same lateral side or not. This step also resulted in 100\% correct identification.

\subsubsection{Lower body plus trunk configurations}

The results of the three steps are again described individually below.

Table 2.3: Correlation coefficients (cc's) used for left and right identification in step 3. The "cc's with"-column indicates the segments - for which it is known whether they are left or right - used for determining the component (third column, constructed with $\mathrm{J} 4.8$ algorithm in Weka) to determine left and right segments.

\begin{tabular}{lll}
\hline Segments & cc's with & component \\
\hline Shoulders & upper arms & $a_{z}$ \\
Forearms & upper arms & $a_{x}$ \\
Hands & forearms & $a_{y}$ \\
Lower Legs & upper legs & $a_{x}$ \\
Feet & lower legs & $a_{x}$ \\
\hline
\end{tabular}




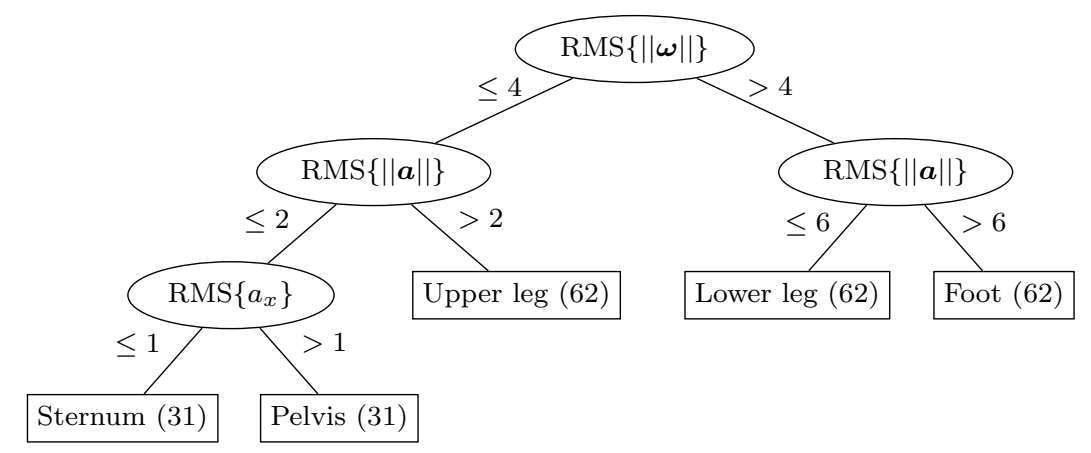

Figure 2.4: Decision tree for segment identification (step 1), when using a lower body plus trunk configuration. 31 walking trials were used $(31 \cdot 8=248$ sensors $)$. 10 fold cross-validation was used for testing the tree, resulting in $248(100 \%)$ correctly classified inertial sensors.

\section{Step one - segment identification}

The decision tree for lower body plus trunk identification is shown in Figure 2.4 To train this tree, 31 walking trials were used $(31 \cdot 8=248$ sensors $)$. 10 -fold cross-validation was used for testing the tree, resulting in 248 (100\%) correctly classified inertial sensors.

\section{Step two - left and right upper arm and upper leg identification}

For left and right upper leg identification the tree from Figure 2.3 can be used again, which resulted in $100 \%$ correctly classified sensors.

\section{Step three - left and right identification for remaining segments}

This step is also the same as the left and right leg identification in the full-body configuration case (see Table 2.3), that is, the correlations of acceleration in $x$ direction between upper and lower legs and between lower legs and feet were used, resulting in $100 \%$ correctly classified sensors.

\subsubsection{Testing the lower body plus trunk identification al- gorithms on the patients}

The decision trees trained using the walking trials of the healthy subjects were tested on the walking trials of the patients, after the ACL reconstruction. This resulted in $100 \%$ correctly identified inertial sensors in all three steps. 


\subsection{Discussion}

The decision trees were trained with features extracted from walking trials involving healthy subjects. It is assumed that the system 'knows' the movement of a subject using for example, movement classification algorithms as described in literature [2, 81. This is important, because for our current method the subject needs to be walking. Our expectation is that the identification will become more robust when combining the current classification method with other daily-life activities. For example, when standing up from sitting the sensors on the upper legs rotate approximately $90^{\circ}$, which make these sensors easy to identify. These other activities could then be monitored using activity classification as described, for example, in [2, 81, provided that this is possible without having to know the segment to which each sensor is attached beforehand. Then, based on this information, the correct decision tree for identifying the sensors can be chosen. Several new features (such as peak count or peak amplitude) will be needed when other activities are investigated.

It is not always essential (or even desirable) to use a full-body configuration, for example the ACL patients, where the interest is mainly on the gait pattern and the progress in the rehabilitation process. If not all the sensors are used, there are two options. The first option is to use a known subset of the 17 inertial sensors and to use decision trees that are trained using this subset of the sensors. This was shown for a lower body plus trunk configuration, but can be done similarly for every desired configuration, using the same methods. If it is not clear which segments are without sensors, the correlation features between different sensors and the ranking can not be used anymore, because these are both dependent on the number of sensors that is used (if for instance the sensors on the feet are missing - and this is not known - the sensors on the lower legs will be classified as if they are on the feet). A second option that can be used in this case, is to use a new decision tree that was created with features of all the 17 inertial sensors, but without the ranking (so using actual RMS and variance values) and without the correlation coefficients between different sensors (on the other hand, inter-axis correlation coefficient could be used, because they are not dependent on other sensors). To demonstrate this, a decision tree was constructed, which resulted in 400 of 527 correctly classified instances $(75.9 \%)$. A possible explanation for this decreased performance could be the fact that because of variations in walking speeds and or arm movements between different walking trials - there is more overlap in the (unranked) features, decreasing the performance of arm and leg identification. This implies that the ranking of the features is a suitable method for reducing the overlap of features between different trials. Another option of minimizing variability between subjects and walking speeds is to normalize the features. We tested this by creating a decision tree with normalized instead of ranked features. This resulted in 461 $(87.5 \%)$ correctly classified sensors.

To obtain an indication of the sensitivity to changes in feature values, for each feature in the decision tree in Figure 2.2, the difference between feature- 
value of each sensor and split-value was calculated. For example, for the feature at the top of the tree, RMS $\{\|\boldsymbol{a}\|\}$, the 17 RMS values were ranked and the splitvalue, that is, the mean RMS of ranks 11 and 12 was calculated. Subsequently, the difference between RMS value of each sensor and split-value was calculated (and normalized for each trial), resulting in a measure for the sensitivity to changes in acceleration. If differences are small, even small changes in acceleration can cause incorrectly classified sensors. These differences were calculated for all eight features used in the decision tree and for all trials. For each sensor the mean, variance, minimum and maximum was calculated. From this we concluded that RMS $\{\|\boldsymbol{a}\|\}$, splitting the sensors on the legs from the other sensors, is not sensitive to changes (in acceleration) and $\operatorname{RMS}\left\{\boldsymbol{\alpha}_{x}\right\}$, splitting the sternum- and shoulder-sensors, is very sensitive to changes (in angular acceleration about the $x$-axis), as can also be concluded from the confusion matrix (Table 2.2) where six sternum-sensors were classified as shoulder-sensors (and one vice versa) and all sensors on the legs were correctly classified.

The measurements used in this study involved placing the inertial sensors on the ideal positions as described in the Xsens MVN user manual to reduce soft tissue artifacts 92 . But what is the influence of the sensor positions on the accuracy of the decision tree? Will the sensors be classified correctly if they are located at different positions? To answer this question a decision tree without the translational acceleration features was investigated, because on a rigid body the angular velocities (so also the angular accelerations) are considered to be the same everywhere on that rigid body. This tree for segment identification resulted in an accuracy of $97.2 \%$ (512 of 527 sensors correctly classified). The tree without the translational accelerations also introduced errors in the left and right identification, for example, the left and right upper arm and upper leg identification both resulted in $60 / 62$ (96.8\%) correctly classified sensors. To gain a better understanding of the influence of the sensor positions, additional measurements are required.

In current motion capture systems, data from several inertial sensors is collected and fused on a PC running an application that calculates segment kinematics and joint angles. This application currently requires information about the position of each sensor, which is handled by labeling each sensor and let the user attach it to the corresponding body segment. The algorithm presented in this chapter can be implemented in this application and take over the responsibility of the correct attachment from the user, with the additional advantage to reduce possible attachment errors. Consequently, the procedure must guarantee a $100 \%$ correct identification, which will not always be the case. Therefore, a solution for this problem could be for the user to perform a visual check via an avatar - representing the subject that is measured - in the running application. If the movement of the avatar does not correspond to the movement of the subject, the subject is asked to walk a few steps to which the identification algorithm can be applied again. In addition to this, the system detects the activity the subject performs and can hence apply the algorithm several times during a measurement and alarm the user if the classifications do 
not fully correspond.

In this study, a decision tree classifier was used resulting in $97.5 \%$ correctly classified sensors. Other classifiers were investigated. For example a support vector machine (SVM) as used by Amini et al. in [1] resulted in 518/527 $(98.3 \%)$ correctly classified sensors when a radial basis function was used with best parameters obtained using cross-validation ("CVParameterSelection" in Weka) [91. Disadvantage, however, is that the resulting parameters of the hyperplanes are not as easy to interpret as decision trees.

Other differences with previous studies, as described in the Introduction, are the number of sensors used. While in [38, 1] respectively 5 and 10 inertial sensors were used, our algorithm provides identification for full-body configurations (17 inertial sensors). Whereas in these previous studies only acceleration features (in sensor coordinates) were used, we also use angular velocities - reducing the influence of the position of the sensor on the segment - and rotated sensor data to a global coordinate frame, for a 3D comparison of movement data from different subjects and allowing left and right identification.

Currently the results are based on three walking cycles. Increasing the trial length (which was possible for most of the recorded trials) did not improve accuracy, whereas a decrease resulted in accuracies of $92.6 \%$ when using two walking cycles and $90.1 \%$ when using one walking cycle (without looking at left and right identification). When using one and a half walking cycle, the accuracy was $92.0 \%$, hence using multiples of full walking cycles seems no necessity.

To test the influence of integration drift on the estimation of the walking direction, we added an error angle to the angle $\theta$ from $(2.5)$. The accelerometer bias stability is $0.02 \mathrm{~m} / \mathrm{s}^{2}$ [93], which can cause a maximum error in velocity of $0.06 \mathrm{~m} / \mathrm{s}$ after integrating over three seconds (the first walking cycle was always within three seconds). This subsequently leads to an error in the angle $\theta$ of 3.5 degrees. We added a random error angle, obtained from a normal distribution with standard deviation of 3.5 degrees to the angle $\theta$. From this we calculated the features and tested them on the decision trees constructed using the normal features. This resulted in $97.7 \%$ correctly classified sensors in step one and $100 \%$ correctly classified sensors in the steps two and three. For an error angle of 10 degrees $97.2 \%$ of the sensors were correctly classified in step one. In steps two and three all sensors were correctly classified, except for the upper legs, from which $96.8 \%$ of the sensors were correctly classified.

No outstanding differences between male and female subjects were observed.

\subsection{Conclusions}

A method for the automatic identification of inertial sensor placement on human body segments has been presented. By comparing 10 easy to extract features, the body segment to which each inertial sensor is attached can be identified with an accuracy of $100.0 \%$ for lower body plus trunk configurations and $97.5 \%$ for full-body configurations, under the following constraints, which 
are satisfied in most practical situations:

- From a standing start (so the initial sensor inclination in the global frame can be obtained) the subject starts walking normally in a straight line, with sufficient arm movement.

- The sensor configuration needs to be known.

The features were extracted from magnitudes and 3D components of accelerations, angular velocities and angular accelerations, after transforming all signals to a global coordinate frame with $x$-axis in walking direction, $y$-axis pointing left and $z$-axis vertical. Identification of left and right limbs was realized using correlations with sternum orientation for upper arms and pelvis orientation for upper legs and for remaining segments by correlations with sensors on adjacent segments. We demonstrated the robustness of the classification method for walking in ACL reconstruction patients.

When the sensor configuration is unknown, the ranking and the correlation coefficients between sensors cannot be used anymore. In this case, only $75.9 \%$ of the sensors are identified correctly (that is 400 of 527 sensors, based on a full body configuration). If it is known which sensors are missing, another decision tree without the missing sensors can be used. If the sensors are not attached to the optimal body positions, decision trees which only use features extracted from angular velocities and angular accelerations can be used instead. 
2. Automatic identification of inertial sensors during walking 


\section{Chapter 3}

\section{On-body inertial sensor location and activity recognition}

口 


\section{Abstract}

In current inertial motion capture systems, the attachment of the sensors to the body segments is often a complex and time consuming task. Each sensor has to be attached to a predefined body segment which makes it prone to errors. In the previous chapter we presented a method for automatically identifying the body segment to which an inertial sensor is attached during walking, by comparing features extracted from accelerometers and gyroscopes of different sensors. In this chapter we present a new method which applies the information of a single inertial sensor to recognize its location on the body and the activity the user is performing.

Logistic regression models were trained using measurements of 10 healthy subjects wearing 17 inertial sensors, performing 18 activities of daily living. The robustness of the models was tested using measurements of walking trials of 15 stroke patients. In a first step we calculate the probability that the user is walking. If this probability is high enough, in a second step the body segment to which the sensor is attached is estimated, again using a probabilistic model. This resulted in an accuracy of $87.2 \%$ for a full-body sensor configuration and $99.3 \%$ when using a lower body plus sternum configuration. A third step is presented for activity recognition, which is useful for time windows after the first two steps. This resulted in accuracies up to $91.3 \%$. 


\subsection{Introduction}

T NERTIAL SENSORS are becoming increasingly popular for human move1 ment analysis. Advantages over traditionally used optical systems are the absence of line of sight problems and the possibility to perform measurements outside of the laboratory 62 . Ambulatory measurements are important for training in sports and performance assessment of patients in an in-home setting [77.

Attaching and connecting the inertial sensors is often a complex and time consuming task, especially for people who do not work with the system on a regular basis. Moreover, it is prone to errors, because each sensor has to be attached to a predefined body segment. Despite the fact that the set-up time for inertial systems is significantly lower (less than 15 minutes for an Xsens MVN Biomech system [93]) than for optical systems [10, it is still a significant amount of time.

Fortunately, decreasing sensor sizes and upcoming wireless inertial sensor technology facilitate easier and faster attachment of the the inertial sensors, for example using Velcro ${ }^{\circledR}$ straps 98 , or even plasters [41]. This could be improved even further by removing the necessity to attach each (wireless) sensor to a predefined segment. Leaving the body segment identification to an automated algorithm, both the set-up time and the number of attachment errors could be reduced.

In previous work 88] we presented a method for identifying the body segment to which inertial sensors are attached during walking. Features, calculated from accelerations, angular velocities and angular accelerations, were ranked after which a decision tree was used for classifying the sensors. A limitation of this method is the requirement to know the sensor configuration beforehand. This method also makes it necessary to run the algorithm on a centralized computer that collects information from all sensors. Another limitation of the algorithm is the assumption that the subject is walking.

Regarding activity recognition many research has been conducted [2] 81, however most of the presented algorithms require the user to place the sensors on a predefined location. Only a few researchers have tried to solve both activity and sensor location recognition simultaneously.

$\mathrm{Xu}$ et al. 94 proposed a sparse signal-based solution, to co-recognize human activity and sensor location in wearable sensor networks. They tested their algorithm in a pilot study with three subjects performing 14 different activities while wearing a triaxial accelerometer and a biaxial gyroscope on seven different on-body locations. Their algorithm consists of feature extraction, followed by sparse representation via $l_{1}$ minimization and Bayesian sparse signal-based classification. They showed that their approach resulted in $87.72 \%$ accuracy. However, several important questions remain. For example, nothing is mentioned about the sensor orientation with respect to the segment. Features were extracted from separate axes of the sensors and therefore it is evident that different results are measured if the sensor is orientated differently on different 
segments. Furthermore, they only showed their results for measurements of three healthy participants. Their algorithm was not tested on patients with movement disorders.

Amini et al. [1] used an unsupervised technique to identify periods in which subjects are walking in long time series data. Once walking activity was discovered, the location of an accelerometer on the human body was estimated using supervised learning by means of a support vector machine. On average their technique achieved $89 \%$ accuracy in estimating the location of accelerometers on six different human body segments. Also several questions remain, first of all, no probabilistic model was used, resulting only in a classification outcome instead of a probability. They also did not test their algorithm on patients with movement disorders.

In this chapter we present a novel iterative method for inertial sensor location and activity recognition. A full-body configuration, that is 17 inertial sensors, is used. In a first step, for each inertial sensor the probability that a user is walking is calculated, independent of where the inertial sensor is located and its orientation with respect to the segment. In a next step, the location of the sensor is identified, provided that the activity that the user is walking is large enough. In a third step the activity is classified, useful for time windows after the first two steps. For all three steps a probabilistic model was trained using supervised learning. For this, measurements of 18 activities of daily living, recorded from ten healthy subjects were used. The robustness of our algorithm was subsequently tested on walking trials of 15 stroke patients.

\subsection{Method}

\subsubsection{Experiments}

From ten healthy subjects (4 female, 6 male, all between 19-32 years old), we recorded 18 activities of daily living using an Xsens MVN Biomech system 93. A full body configuration was used, that is, 17 inertial sensors were placed on the head, sternum, shoulders, pelvis, upper arms, forearms, hands, upper legs, lower legs and feet 92. Data from the inertial sensors - that is, 3D measured acceleration $\left(\boldsymbol{s}^{s}\right)$ and 3D angular velocity $\left(\boldsymbol{\omega}^{s}\right)$, both expressed in sensor coordinate frame - recorded with a sampling frequency of $120 \mathrm{~Hz}$ was saved in MVN file format, converted to XML and loaded into MATLAB ${ }^{\circledR}$ for further analysis.

Walking at different speeds in a straight line and with turns, sit to stand, stand to sit, laying down, walking stairs up and down, jumping, cycling, sitting at a table and working were recorded. Also combined series of activities for example sitting, standing up, walking, opening a door, grab an object in another room and going back - were recorded. All activities were recorded three times, except cycling in one subject, who was not able to ride a bike.

The research was in full compliance with the "Declaration of Helsinki" and 
written informed consent was obtained from all subjects for publication of the results.

To test the robustness of our algorithms, walking trials of 15 stroke patients measured during the EU FP7 project INTERACTION [77] were used. These trials were also recorded using an Xsens MVN Biomech system with sampling frequency of $120 \mathrm{~Hz}$. This study was approved by the local medical ethical committee (number METC/13016.MEU).

\subsubsection{Sensor location and activity recognition method}

The identification of the inertial sensors consists of three parts: preprocessing, feature extraction and classification.

\section{Preprocessing}

To improve comparison over different body segments and subjects, inertial sensor signals were preprocessed before being offered to the classification algorithm. In short this means all signals are rotated to a coordinate frame with all $z$ axes aligned. This way the algorithm is invariant for the orientation of a sensor with respect to a segment. We assume a natural standing posture before walking. During this standing posture the initial sensor inclination was estimated from the measured acceleration and the angular velocity bias was estimated from the gyroscope signals (averaging over 120 samples). When the subjects were moving, the change of orientation was estimated by integrating the angular velocities after bias correction. See 88 for more details about this approach. Next, the signals were rotated to a coordinate system with the $z$-axis defined as the vertical axis, aligned with gravity. The gravitational acceleration was subtracted from the $z$-component of the accelerometer signals, leaving only the acceleration due to motion $\boldsymbol{a}$. No changes were made to the horizontal axis of each sensor and therefore we can still not use information from signals of these axes, since these axes could be aligned differently with respect to the segment for different subjects. However, we can extract features from the magnitude of these ( $x$ and $y$ ) axes. To obtain additional information about (rotational) accelerations, which are invariant to the position of the sensor on the segment, the 3D angular acceleration $\boldsymbol{\alpha}$ was calculated:

$$
\boldsymbol{\alpha}=\frac{d \boldsymbol{\omega}}{d t} .
$$

\section{Feature extraction}

Using MATLAB ${ }^{\circledR}$, features were extracted from the different signals. These features were mean, root mean square, variance, minimum and maximum and they were calculated for the:

- magnitude of 3D $\boldsymbol{a}, \boldsymbol{\omega}$ and $\boldsymbol{\alpha}$ (i.e. for $\|\boldsymbol{a}\|,\|\boldsymbol{\omega}\|$ and $\|\boldsymbol{\alpha}\|$ )

- z-component of $\boldsymbol{a}, \boldsymbol{\omega}$ and $\boldsymbol{\alpha}$ (i.e. for $a_{z}, \omega_{z}$ and $\alpha_{z}$ ) 
- magnitude of the $x$ - and $y$-axes of $\boldsymbol{a}, \boldsymbol{\omega}$ and $\boldsymbol{\alpha}$ (i.e. for $\sqrt{a_{x}^{2}+a_{y}^{2}}$, $\sqrt{\omega_{x}^{2}+\omega_{y}^{2}}$ and $\left.\sqrt{\alpha_{x}^{2}+\alpha_{y}^{2}}\right)$

\section{Classification}

For classification, the extracted features were subsequently used in Weka (Waikato Environment for Knowledge Analysis) - a collection of machine learning algorithms for data mining tasks [25, 89]. Since we are interested in probabilities for different classes, linear logistic regression was used for classification 39. In Weka the SimpleLogistic function was used for this classification. In SimpleLogistic, the LogitBoost algorithm is used for fitting logistic models of the form

$$
\operatorname{Pr}(G=j \mid X=x)=\frac{e^{F_{j}(x)}}{\sum_{k=1}^{J} e^{F_{k}(x)}}
$$

where $\operatorname{Pr}$ is the class probability for class $j$ and $F_{j}(x)=\beta_{j}^{T} \cdot x$ are linear regression functions with $x$ the input vector. More details can be found in 39].

The models were trained in Weka and tested using 10-fold cross validation, because in the literature this has been shown to be a good estimate of the error rate for many problems 91 .

We started with simultaneous classification of both the activity and the sensor location, by using 18 (activities) multiplied with 10 (locations) different classes. However, since this did not lead to good results, we decided to use an iterative approach.

For robust classification of both the sensor location and the activity the user is performing, three consecutive classification steps were designed, starting with walking recognition. We start with walking recognition, because in many applications the user always walks eventually and most segments move very distinctively, making it ideal for sensor location recognition in a second step. Furthermore, this two-class approach is a robust starting point in this situation, where both the activity and the sensor location are unknown. Once it is recognized that a user is walking in a certain time window, the location of a sensor is estimated in a second classification step. After all sensor locations are recognized, a third and final step can be used to recognize the activity the user is performing, in time windows after the first two steps.

Step 1: Walking recognition In this first step the activity is classified. 17 different activities were used for this, all except the cycling measurements. We left this out, because these measurements started with standing next to the bike and sometimes the subject had to walk a few steps towards the bike. In general, when putting on sensors it is unlikely to be in a position to start cycling immediately. For a correct training (and testing) process - that is, 
to be sure only one activity is performed during each labeled instance - the features were extracted from three second windows, after the subject started movement. Start of movement was obtained by thresholding the summation of magnitudes of accelerations and angular velocities $\left(\sum_{i=1}^{n}\left(\left\|\boldsymbol{a}_{i}\right\|_{2}+\left\|\boldsymbol{\omega}_{i}\right\|_{2}\right)\right.$, where $n$ is the number of sensors) of all 17 sensors.

All instances were labeled walking or not-walking depending on the activity as shown in Table 3.1 .

In addition, features were extracted from the period from the start of the measurement until the start of the movement detection. These are the moments in which the subject is sitting or standing and is about to start with an activity. These time windows were labeled not-walking, in order for a better classification of moments in which a subject is not walking. Only features extracted from the magnitude of the accelerations, angular velocities and angular accelerations were used in this step, because the subjects are not always standing at the beginning of a measurement. Therefore the preprocessing steps can not be applied here.

Step 2: Sensor location recognition In this step, the location of the sensors is classified under the assumption that a subject is walking, which was predicted in the previous step. For this the same methods as described for step one are used, but now with 45 features, as described in Section 3.2.2. For this step only the trials are used in which the subjects were walking. The features are extracted from three second windows after the subject started walking. The sensors are labeled in ten different classes, being pelvis, sternum, head,

Table 3.1: All activities and the class labels that were used for step 1: walking recognition and for step 3: activity recognition.

\begin{tabular}{lll}
\hline & \multicolumn{2}{c}{ Class label } \\
\cline { 2 - 3 } Activity & Step 1 & Step 3 \\
\hline Straight walking slowly & walking & walking \\
Straight walking normally & walking & walking \\
Straight walking quickly & walking & walking \\
Walking with turns slowly & walking & walking \\
Walking with turns normally & walking & walking \\
Walking with turns quickly & walking & walking \\
Sit to stand & not-walking & sit-to-stand \\
Stand to sit & not-walking & stand-to-sit \\
Sit, stand, walk, stand, sit & not-walking & - \\
Using computer while sitting & not-walking & sit-or-stand-and-work \\
Writing while sitting & not-walking & sit-or-stand-and-work \\
Writing while standing & not-walking & sit-or-stand-and-work \\
Opening door and grasp objects & not-walking & - \\
Laying down & not-walking & laying-down \\
Jumping & not-walking & jumping \\
Walking stairs down & walking & walking \\
Walking stairs up & walking & walking \\
\hline
\end{tabular}


Table 3.2: Confusion matrix for step 1: walking recognition, when using a full-body configuration (17 inertial sensors). Results are from 10 healthy subjects performing 3 times 17 activities using 10-fold cross-validation. 15117 of a total of 17340 instances were correctly classified $(87.2 \%)$. Note that the specificity (the proportion not-walking classified as such) is very high (94.6\%), which is good since these time windows are then not used for location classification in the next step.

\begin{tabular}{rrl}
\hline $\mathrm{a}$ & $\mathrm{b}$ & $<-$ classified as \\
\hline 2576 & 1504 & $\mathrm{a}=$ walking \\
719 & 12541 & $\mathrm{~b}=$ not-walking \\
\hline
\end{tabular}

Table 3.3: Confusion matrix for step 1: walking recognition, when a lower body plus sternum configuration ( 8 inertial sensors) is used. Results are from 10 healthy subjects performing 3 times 17 activities using 10-fold cross-validation. 7347 of a total of 8160 instances were correctly classified (90.0\%).

\begin{tabular}{rrl}
\hline $\mathrm{a}$ & $\mathrm{b}$ & $<-$ classified as \\
\hline 1405 & 515 & $\mathrm{a}=$ walking \\
298 & 5942 & $\mathrm{~b}=$ not-walking \\
\hline
\end{tabular}

shoulder, upper arm, forearm, hand, upper leg, lower leg and foot.

Step 3: Activity recognition In this third and last step, the activity is recognized for each of the ten sensor location as identified in the previous step. All instances were labeled in six classes as shown in Table 3.1. The combined activities were left out, since these can not be categorized into one single activity. Again only the 15 features extracted from the magnitudes of 3D $\boldsymbol{a}, \boldsymbol{\omega}$ and $\boldsymbol{\alpha}$ were used here, as described in step 1.

\subsection{Results}

\subsubsection{Step 1: Walking recognition}

The confusion matrix for walking recognition is shown in Table 3.2. From 17340 instances, 15117 were correctly classified $(87.2 \%)$. The specificity (the proportion not-walking classified as such) is very high $(94.6 \%)$, which is good since these time windows are then not used for sensor location classification in step 2 .

Because in most clinical applications a full body configuration is not necessary (or even desirable), we also looked into a configuration with fewer sensors. That is, a lower-body plus sternum configuration, which is a more commonly used configuration in a rehabilitation setting, as we also described in [88. In this configuration eight sensors are used, namely on the pelvis, sternum, upper legs, lower legs and feet. For the walking and not-walking classes a model was 
Table 3.4: Confusion matrix for step 2: sensor location recognition. The model was trained using all walking activities, of ten healthy subjects using 45 features extracted from 3 s time windows. After testing using 10-fold-cross validation 2667 of 3060 sensors were correctly classified $(87.2 \%)$.

\begin{tabular}{ccccccccccl}
\hline $\mathrm{a}$ & $\mathrm{b}$ & $\mathrm{c}$ & $\mathrm{d}$ & $\mathrm{e}$ & $\mathrm{f}$ & $\mathrm{g}$ & $\mathrm{h}$ & $\mathrm{i}$ & $\mathrm{j}$ & $<$ - classified as \\
\hline 169 & 1 & 0 & 5 & 4 & 0 & 1 & 0 & 0 & 0 & $\mathrm{a}=$ Pelvis \\
1 & 80 & 13 & 86 & 0 & 0 & 0 & 0 & 0 & 0 & $\mathrm{~b}=$ Sternum \\
0 & 17 & 137 & 20 & 3 & 1 & 2 & 0 & 0 & 0 & $\mathrm{c}=$ Head \\
3 & 36 & 5 & 316 & 0 & 0 & 0 & 0 & 0 & 0 & $\mathrm{~d}=$ Shoulder \\
1 & 0 & 0 & 8 & 336 & 14 & 1 & 0 & 0 & 0 & $\mathrm{e}=$ UpperArm \\
0 & 1 & 3 & 1 & 34 & 280 & 39 & 2 & 0 & 0 & $\mathrm{f}=$ Forearm \\
2 & 1 & 2 & 0 & 5 & 57 & 289 & 4 & 0 & 0 & $\mathrm{~g}=$ Hand \\
2 & 0 & 0 & 0 & 0 & 5 & 5 & 346 & 1 & 1 & $\mathrm{~h}=$ UpperLeg \\
0 & 0 & 0 & 0 & 0 & 0 & 1 & 0 & 358 & 1 & $\mathrm{i}=$ LowerLeg \\
0 & 0 & 2 & 0 & 1 & 0 & 0 & 0 & 1 & 356 & $\mathrm{j}=$ Foot \\
\hline
\end{tabular}

again trained and tested using the healthy subjects. The confusion matrix of this is shown in Table 3.3. In this case, from 8160 instances, 7347 were correctly classified $(90.0 \%)$.

\subsubsection{Step 2: Sensor location recognition}

The confusion matrix for the sensor location classification is shown in Table 3.4 2667 of 3060 sensors were correctly classified $(87.2 \%)$. When looking at the confusion matrix it can be noticed that most of the incorrectly classified sensors are the ones on the upper body, especially sternum and shoulders and forearms and hands are interchanged, which can be explained because these sensors move similar during walking.

For the lower body plus sternum configuration the confusion matrix, after testing the model with ten-fold cross-validation is shown in Table 3.5. From 1440 sensors, 1430 were correctly classified (99.3\%).

\subsubsection{Step 3: Activity recognition}

The average activity classification accuracy for 10 on body locations is $86.8 \%$. The sensor on the pelvis receives the largest activity classification accuracy, 411 of a total of 450 instances are correctly classified in this case (91.3\%). The sensors with the lowest accuracy were the ones on the feet $(84.4 \%)$. For these sensors the activities sit-to-stand, stand-to-sit and sit-or-stand-and-work are interchanged a lot. This is expected, because these sensors move less or are not moving at all during these activities.

\subsubsection{Testing the models on stroke patients}

The models from step 1 and 2, for a lower body plus sternum configuration were subsequently tested on 15 stroke patients. First the walking detection 


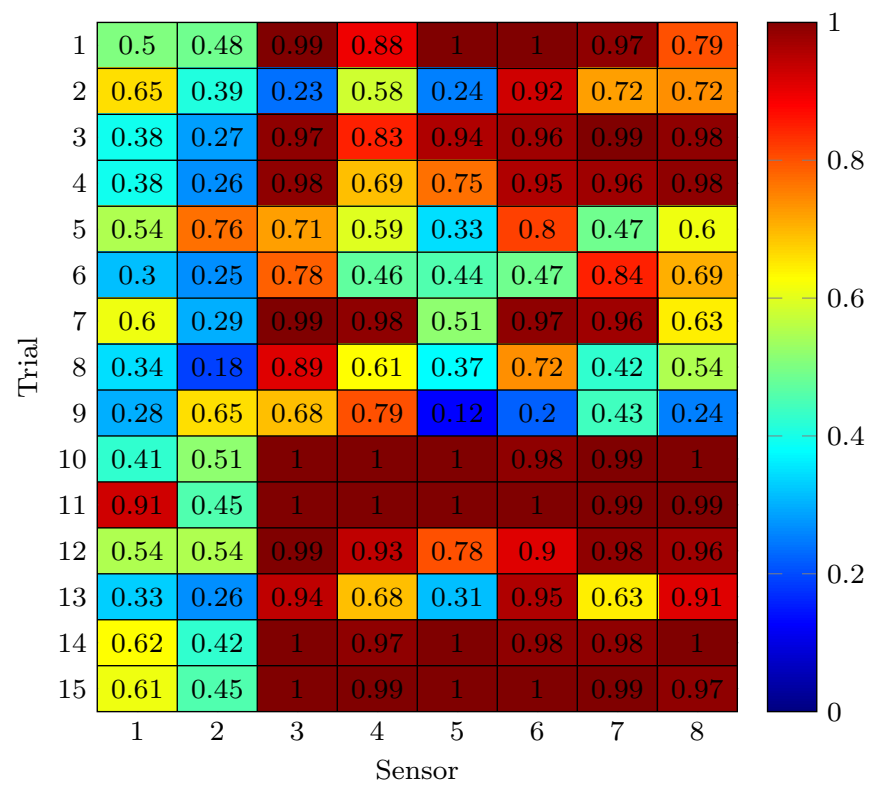

Figure 3.1: Probabilities of an instance (sensor) belonging to the class walking. These are the results for the walking trials of the 15 stroke patients, which in theory means all probabilities should be greater than 0.5 . The sensor numbers are $1=$ pelvis, $2=$ sternum, 3,6=upper leg, 4,7=lower leg and 5,8=foot. Notice that for the pelvis and sternum sensors the probabilities are lower, making it harder to classify whether a subject is walking or not based on these sensor locations. 


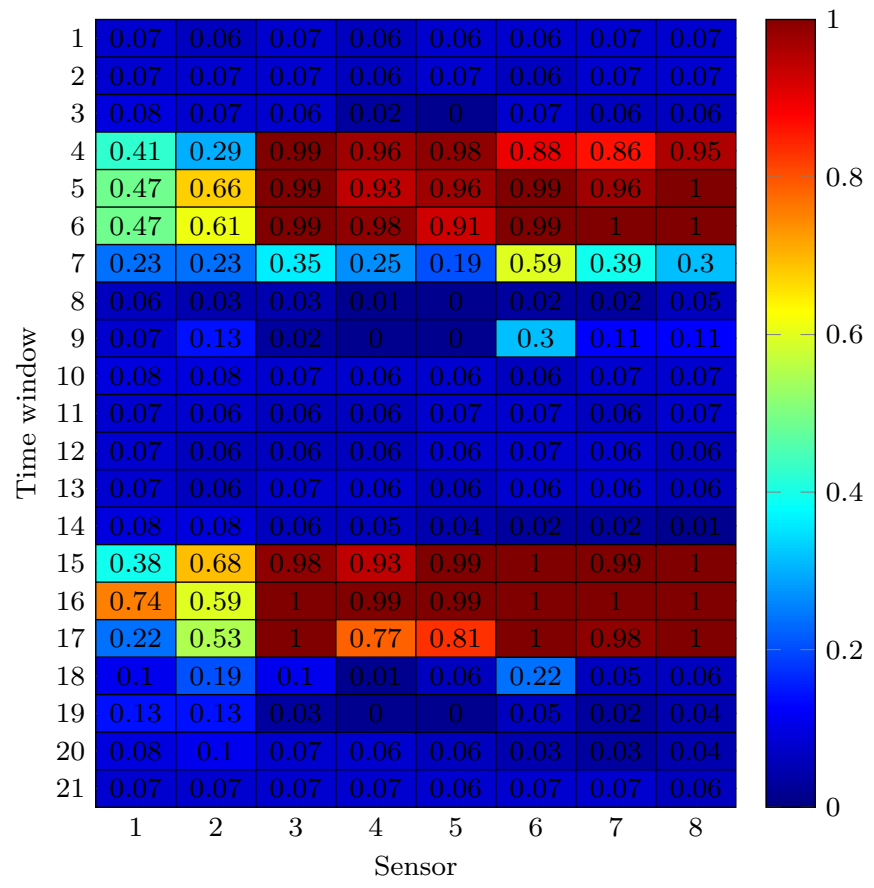

Figure 3.2: Probabilities of an instance (sensor) belonging to the class walking. These are the results for different time windows (of each $3 \mathrm{~s}$ ) of a walking trial of a stroke patient (trial 3 in Figure 3.1. 
Table 3.5: Confusion matrix for step 2: sensor location recognition, when using a lower body plus sternum configuration. The model was again trained using all walking activities, of ten healthy subjects using 45 features extracted from $3 \mathrm{~s}$ time windows. After testing using 10-fold-cross validation 1430 of 1440 sensors were correctly classified $(99.3 \%)$.

\begin{tabular}{cccccl}
\hline $\mathrm{a}$ & $\mathrm{b}$ & $\mathrm{c}$ & $\mathrm{d}$ & $\mathrm{e}$ & $<-$ classified as \\
\hline 178 & 2 & 0 & 0 & 0 & $\mathrm{a}=$ Pelvis \\
0 & 180 & 0 & 0 & 0 & $\mathrm{~b}=$ Sternum \\
0 & 0 & 359 & 1 & 0 & $\mathrm{c}=$ UpperLeg \\
0 & 0 & 3 & 357 & 0 & $\mathrm{~d}=$ LowerLeg \\
0 & 1 & 0 & 3 & 356 & $\mathrm{e}=$ Foot \\
\hline
\end{tabular}

Table 3.6: Confusion matrix for step 2: sensor location recognition, when using a lower body plus sternum configuration. The model was trained using all walking activities, of ten healthy subjects using 45 features extracted from $3 \mathrm{~s}$ time windows and tested on walking trials of 15 stroke patients. 94 of 120 sensors were correctly classified $(78.3 \%)$.

\begin{tabular}{cccccl}
\hline $\mathrm{a}$ & $\mathrm{b}$ & $\mathrm{c}$ & $\mathrm{d}$ & $\mathrm{e}$ & $<$ - classified as \\
\hline 14 & 0 & 1 & 0 & 0 & $\mathrm{a}=$ Pelvis \\
4 & 10 & 1 & 0 & 0 & $\mathrm{~b}=$ Sternum \\
0 & 0 & 29 & 1 & 0 & $\mathrm{c}=$ UpperLeg \\
0 & 0 & 8 & 22 & 0 & $\mathrm{~d}=$ LowerLeg \\
0 & 9 & 0 & 2 & 19 & $\mathrm{e}=$ Foot \\
\hline
\end{tabular}

model was tested on the stroke patients. From 120 instances only 76 were correctly classified (63.3\%). We noticed however that most of the sensors that were incorrectly classified were located on the pelvis and the sternum, as can be seen in Figure 3.1. Since we have only two classes, a probability larger than 0.5 means that an instance belongs to the class walking and hence, based on this instance it is assumed the subject is walking. Since we know in this specific case that all sensors on a row belong to the same walking trial we calculated the mean of these probabilities. From the mean probability of each row now 12 of the 15 rows are larger than $0.5(80 \%)$. A similar plot can be made for a single walking trial with on each row the probability calculated for each three-second time window. This is shown in Figure 3.2. This is a measurement in which the patient stands still, walks ten meter, turns and walks back. From this figure the two walking periods can be observed easily (the larger probabilities, i.e. red color), making it possible to classify the sensor locations during those moments. Alternatively, one could wait until the probability that a single sensor belongs to the class walking is larger than 0.5 , which would be in time window 16 in this case. Above steps could also be done for the full-body configuration, however for clarity of the plots, we chose to show the lower body plus sternum 
Table 3.7: Overview of all results. The selectivity and specifity are only shown for step 1, since the classification conists of two classes here. FB=Full body configuration, $\mathrm{LBS}=$ Lower body plus sternum configuration

\begin{tabular}{lrrr}
\hline & Step 1, walking recognition & \\
Scenario & Accuracy & Selectivity & Specificity \\
\hline FB, healthy & $87.2 \%$ & $63.1 \%$ & $94.6 \%$ \\
FB, patients & $72.9 \%$ & $72.9 \%$ & - \\
LBS, healthy & $90.0 \%$ & $73.2 \%$ & $95.2 \%$ \\
LBS, patients & $63.3 \%$ & $63.3 \%$ & - \\
\hline
\end{tabular}

Step 2, sensor location recognition

\begin{tabular}{lr} 
Scenario & Accuracy \\
\hline FB, healthy & $87.2 \%$ \\
FB, patients & $61.6 \%$ \\
LBS, healthy & $99.3 \%$ \\
LBS, patients & $78.3 \%$ \\
\hline
\end{tabular}

Step 3, activity recognition

\begin{tabular}{lr} 
Scenario & Accuracy \\
\hline FB, healthy (average) & $86.8 \%$ \\
FB, healthy (min, foot) & $84.4 \%$ \\
FB, healthy (max, pelvis) & $91.3 \%$
\end{tabular}

configuration here.

The model for sensor location classification that was trained on the healthy subjects was next tested on the 15 stroke patients of which the confusion matrix is shown in Table 3.6. Now, 94 of 120 sensors were correctly classified (78.3\%).

The full-body configuration was also investigated. The model from Table 3.4 trained on the healthy subjects was tested on the walking trials of the 15 stroke patients. The confusion matrix is not shown due to space limitation, but $157(61.6 \%)$ of a total of 255 were correctly classified. Especially the sternum and shoulders, and the forearms and hands are mixed up, as we already noticed in the results of the healthy subjects.

All results are summarized in Table 3.7

\subsection{Discussion}

We presented an iterative method for sensor location classification and activity recognition for full-body inertial sensor configurations. We showed the robustness of our method by testing the models on walking trials of 15 stroke patients. Our method can be used for applications with varying numbers of sensors and even using a single sensor or for example on a smart phone, because no information from other sensors (or correlations between sensors) is required. 
In most clinical cases a full-body configuration is not required or even desirable, for practical, time and costs reasons. In most cases, especially for gait analysis, a lower body plus sternum configuration suffices, as we also described in our previous study 88, where we investigated subjects after anterior cruciate ligament reconstruction. The sternum sensor is in this case useful for assessing the balance of the subject. Therefore we also showed results, when a lower body plus sternum configuration is used.

Before starting this study, we used the 31 walking trials of ten healthy subjects that were used in 88 to test the logistic regression classification performance. For this comparison, we used the features as they were calculated in the previous study [88. However, since we want to classify the location using a single sensor, we left out the correlations between sensors and the ranking of the features. That is, RMS, variance, maximum and inter-axis correlation coefficients were used. The model was trained on the full-body configuration of these trials and tested using cross validation. In addition a model was trained using a lower-body plus sternum configuration which was also tested on measurements of 7 patients after anterior cruciate ligament (ACL) reconstruction. The results for a full-body configuration after testing using 10-fold cross-validation are that 482 of 527 body segments are correctly classified (91.5\%). For a lower-body plus sternum configuration 239 of a total of 248 body segments are correctly classified (96.4\%). In the ACL patients 133 of the 136 body segments were correctly classified $(97.8 \%)$. These results are comparable to the results described in this chapter.

As described in the method section, we also investigated the activity and location classification in a single step. Therefore, a classifier was trained with all location/activity combinations as classes, giving 18 activities times 10 sensor locations. For these 180 combined location/activity classes 4317 of a total of 9112 instances were correctly classified (47.4\%). Since this is not a result we were satisfied with, we decided to use an iterative approach.

Regarding the walking recognition and the activity classification, now 15 features were used that were extracted from magnitudes of acceleration, angular velocity and angular acceleration. We expect that a higher accuracy can be achieved if features from $z$ axes and magnitude of $x$ and $y$ axes are also used, however then the assumption has to be made that a subject is standing at a certain moment in order to estimate the sensor inclination based on the accelerometer signal that measures gravitational acceleration only in this case. This standing detection could be implemented after walking is detected, by selecting the period directly before or after walking when the subject is not moving. Therefore we only used the features of the $z$ axes (and magnitude of $x$ and $y$ axes) for the location classification models, in the step after walking is recognized.

We chose to use only time domain features and not frequency domain features, for example FFT, because frequency domain features require more elaborate calculations. Using only time domain features the algorithm can run on moderate hardware currently present in a typical sensor or for example on a 
mobile device with an inertial sensor, for example a mobile phone.

Features were extracted from time windows of three seconds. We also looked into windows of different lengths. A longer time window resulted in higher accuracy, however the chance that a subject does more than one activity also increases if the time windows are larger. Besides in three seconds we assumed most subjects are able to complete a full walking cycle and therefore the classification should work with features extracted from only three second windows. In our study (for example Figure 3.2 we looked at successive time windows. Depending on the processing power that is available, an alternative could be the use of overlapping or sliding windows, delivering a higher time resolution.

An additional advantage of our probabilistic approach is that instead of using information from one time window, it is also possible to calculate and compare probabilities for different (subsequent) time windows (provided that the subject is still walking) of a trial and use the assumption that a sensor stays attached to the same body segment. The probabilities we showed in Figure 3.2 regarding an instance belonging to the class walking are also calculated for the sensor location recognition. Similarly, one could wait until the probability of a sensor belonging to a certain class is large enough to reliably say that this sensor is located on this particular segment.

For the measurements in this study we placed the inertial sensors on the locations that were specified in the Xsens MVN user manual 92. The results could change if the sensors are placed on the segment on a different location. To reduce dependencies in this, one could however use features extracted from only angular velocity and angular acceleration, since these are the same everywhere on a rigid body.

In this study we did not look into the distinction between left and right for the sensor location identification. An additional step, as described in 88, could be added for this after the currently presented methods. This would require additional heading information, obtained for example from the walking direction. Also correlations between sensors are required [88].

\subsection{Conclusion}

We presented a novel method for identifying the segment to which an inertial sensor is attached. In a first step, for each inertial sensor the probability that a user is walking is calculated, independent of where the inertial sensor is located. Once the probability that a subject is walking is large enough, the location of the sensor can be classified in a next step, again using a probabilistic model. This resulted in an accuracy of $87.2 \%$ for a full-body sensor configuration and $99.3 \%$ when using a lower body plus sternum configuration. After the location is recognized, a third step is presented for activity recognition, which resulted in accuracies up to $91.3 \%$. 


\section{Chapter 4}

\section{Ultrasonic range measurements on the human body}

口

Published as:

D. Weenk, B. J. F. van Beijnum, A. Droog, H. J. Hermens, P. H. Veltink Ultrasonic range measurements on the human body Seventh International Conference on Sensing Technology, ICST 2013, 3-5 Dec 2013, Wellington, New Zealand. pp. 151-156 http://dx.doi.org/10.1109/ICSensT.2013.6727633 


\section{Abstract}

Ambulatory range estimation on the human body is important for the assessment of the performance of upper- and lower limb tasks outside a laboratory. In this chapter an ultrasound sensor for estimating ranges on the human body is presented and validated during gait. The distance between the feet is estimated based on the time of flight and compared to an optical reference. The signal to noise ratio of the received signal is used as a measure for the uncertainty of the range estimate. For example when rejecting distance estimates with a signal to noise ratio smaller than 5 , the mean absolute distance difference between the ultrasound sensor and an optical reference system is $7.0 \mathrm{~mm}$ ( $\mathrm{sd} 7.1 \mathrm{~mm}$ ) over six walking trials. 


\subsection{Introduction}

$\mathrm{O}$

N-BODY range measurements are important for assessing the performance of upper- and lower limb tasks [13, 65. Traditionally, force plates in combination with optical systems are used for this. These systems calculate $3 \mathrm{D}$ positions of several markers on the body. Disadvantage of these systems is that measurements are restricted to a lab with cameras, which limits the capture area to a few walking cycles. Ambulatory systems, using for instance inertial sensors, are becoming increasingly popular for tracking human movement. Measurements can be performed outside a lab and even at home, without restrictions in measurement volume, allowing the measurement of different daily-life activities. However, with inertial sensors, relative positions of body segments cannot be measured directly, but are estimated from segment orientations in combination with their lengths 62 . This leads to errors when segment lengths are incorrectly measured or estimated.

Roetenberg et al. [59] investigated a portable magnetic position and orientation tracker, with promising results. By using a 3D magnetic source, positioned on the back of the body, and 3D magnetic sensors placed at different body segments, the accuracy was approximately $8 \mathrm{~mm}$ in position and $5^{\circ}$ in orientation during movement. Disadvantage is the relatively large size and weight $(21 \mathrm{~cm}$ diameter, $11 \mathrm{~cm}$ height, $450 \mathrm{~g}$ ), making it unsuitable for placing it on a foot.

Schepers et al. [70] used shoes instrumented with force/moment and inertial sensors for the ambulatory assessment of foot placement during walking. Lateral foot placement and stride length were estimated from integration of inertial sensor signals (angular velocities and accelerations). However, this method leads to large position errors (drift) after integration. Besides, only position changes are calculated and since the begin positions are unknown this does not result in relative positions of the feet.

Huitema et al. 30] described an ultrasonic motion analysis system, capable of measuring important human gait parameters. Two small ultrasonic receivers were attached to both shoes, while a transmitter was placed stationary on the floor. By subtracting the positions of the feet with zero velocity, step and stride lengths were estimated. Disadvantage is the transmitter placed on the floor, limiting the maximum measurement distance to $8.6 \mathrm{~m}$.

In this chapter the design and validation of an ambulatory ultrasonic onbody range measurement-system is described. We selected ultrasound transducers because they are small and light-weight, making them suitable for placing them on several human body segments. In addition they are low cost and not affected by ferromagnetic materials (especially in floor when walking), as is the case when using magnetic position estimation. 


\subsection{Design of the sensor}

The ultrasound sensor is based on estimating the time of flight $\left(t_{T o F}\right)$. This is in our case the time it takes for an ultrasound signal to travel from a transmitter to a receiver. When this time is known, it can be multiplied with the speed of sound $\left(v_{s}\right)$ to get the distance,

$$
d_{\text {ultrasound }}=v_{s} \cdot t_{T o F} \cdot
$$

The speed of sound is temperature dependent and in air the expression is

$$
v_{s}=331.4 \cdot\left(1+1.83 \cdot 10^{-3} \cdot T_{C}\right),
$$

with $T_{C}$ the temperature in ${ }^{\circ} \mathrm{C}$ [57].

In the remainder of this Section the estimation of the time of flight is described, from the signal processing to the used hardware.

\subsubsection{Time of flight estimation}

A typical example of a received and amplified ultrasound signal is shown in Fig. 4.1 (top), the center frequency of the signal is $40 \mathrm{kHz}$.

The signal strength has a negative correlation with the time of flight and is therefore estimated by calculating the root-mean-square (RMS), after removing the offset. Recorded noise at the beginning of the signal, which is not related to the transmitted pulse, is eliminated (Fig. 4.1) by overwriting these samples with the mean of the signal. For signals with large RMS, nothing is eliminated, to be able to detect small time of flights as well.

To filter out noise - for example, picked up in the circuit between the receiver and the microcontroller - the recorded signal is band-pass filtered using an 8th order zero-phase Butterworth filter with cut-off frequencies 35 and 45 $\mathrm{kHz}$. The amplifier is tuned to be able to pick up also weak signals when the transducers are approximately $90 \mathrm{~cm}$ apart. This leads to a clipping signal when the transducers are close together, causing the slope of the envelope to be steeper than normal (larger frequency than $40 \mathrm{kHz}$ ). Therefore, we make an exception to the band-pass filter when this occurs. When the transducers are close together, which is estimated using the RMS, only a high-pass filter is applied.

To be able to accurately estimate the start of the received pulse (Fig. 4.1. top), and thus the time of flight, the filtered signal is normalized and its envelope is calculated by rectifying and low-pass filtering $(10 \mathrm{kHz}, 4$ th order zero-phase Butterworth filter), see Fig. 4.1 (bottom).

Subsequently, the time of flight $\left(t_{T o F}\right)$ is estimated from the envelope using two successive thresholds, A and B (Fig. 4.1. bottom). Multiplying by the speed of sound $\left(v_{s}\right)$ this gives a first estimate of the range, see (4.1). This range is re-calibrated using a calibration board, as will be described later. 


\subsubsection{Hardware}

The schematic of the sensor is shown in Fig. 4.2. The central part of this ultrasonic range measurement system is an ATmega328P microcontroller running at $14.7456 \mathrm{MHz}$. The processor will output one pulse of a certain width to the gate of the FET (F1), and during that (short) time a current will flow through the primary windings of the transformer (T1), this will induce a high voltage at the secondary side that will excite the transmit transducer (400ST120). Due to its mechanical and electrical properties in combination with the frequency tuned transformer, the transducer will output several in amplitude drastically diminishing pulses $(40 \mathrm{kHz})$. As soon as the pulse to the transformer has been generated, the controller starts converting and storing the signal coming from the amplifier. For this purpose the processor is using a 10 bits Analog to Digital Converter, sampling at $195 \mathrm{kHz}$, of which only the HI byte is used to save on
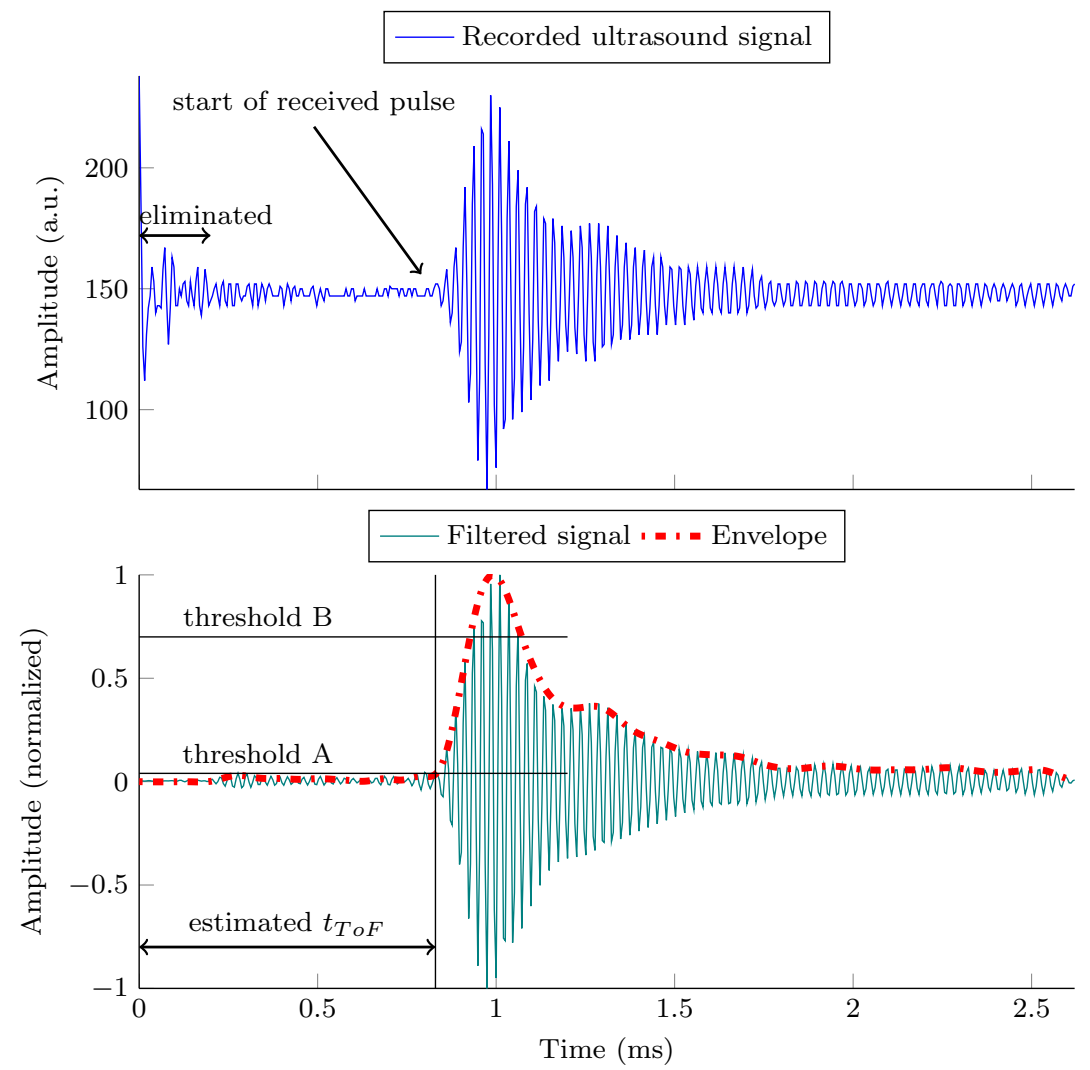

Figure 4.1: Typical example of a recorded ultrasound signal (top). After eliminating the first samples (that are not related to the sent pulse), the signal is band-pass filtered and normalized and the envelope is calculated (bottom). From the envelope the time of flight is estimated using two successive thresholds (A and B). 
time and storage. For storage 512 bytes are used, which is sufficient to measure a range up to $\pm 90 \mathrm{~cm}$. The receive transducer (400SR120) is excited by the acoustic signal sent from the transmitter. The amplifier consists mainly of an LM386 low voltage audio power amplifier. Potentiometer P1 is used for setting the sensitivity while P2 is used for scaling the output signal of the amplifier (powered from $5 \mathrm{~V}$ ) to the input of the Analog to Digital Converter in the ATmega328P microcontroller (powered from 3.3 V). When 512 samples have been taken, these samples are sent via a Bluetooth connection to a computer where the distance is calculated using MATLAB ${ }^{\circledR}$. The Bluetooth module is powered by $3.3 \mathrm{~V}$ and is directly controlled by the microcontroller with a baud rate of $230 \mathrm{k} 4$ bits per second. After the 512 bytes of data is sent to the computer, the controller starts over again with generating the pulse to the gate of the FET.

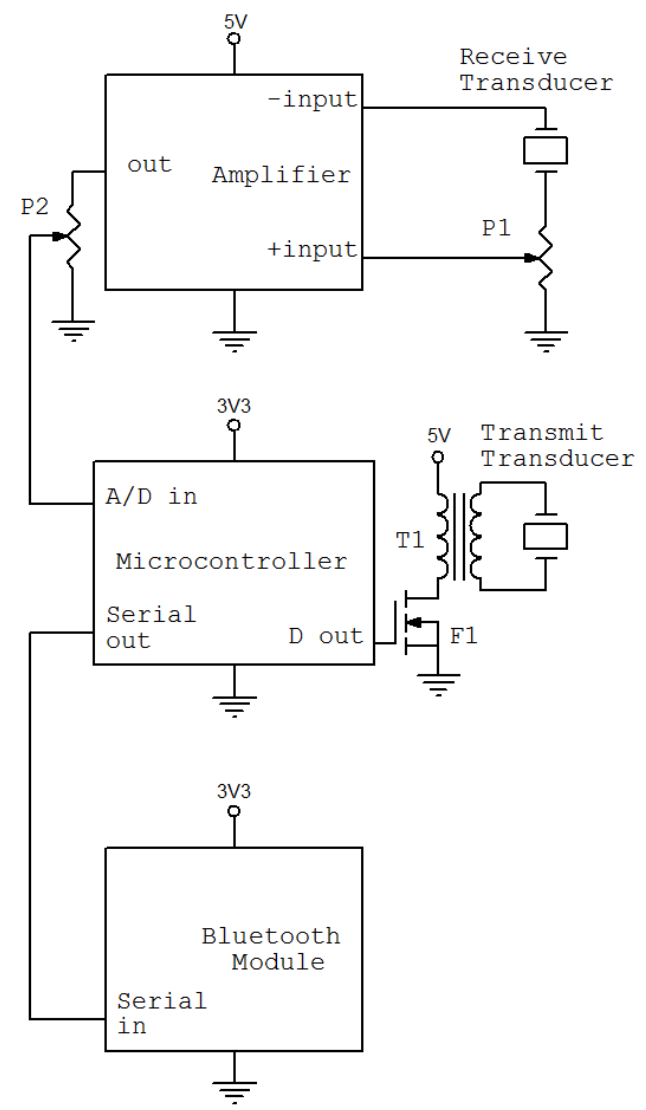

Figure 4.2: Schematic of the ultrasound sensor. The transmit transducer including transformer is placed on one foot, while the receive transducer and the amplifier are placed on the other foot, see Fig. 4.4 The microcontroller and bluetooth module are placed at the waist of the subject. 
This occurs approximately 13.3 times per second.

\subsection{Validation methods}

To validate the ultrasound sensor, the estimated distance was compared to an optical reference system.

\subsubsection{Set-up}

The Xsens ForceShoes (Xsens Technologies B.V. 93]), containing two 6D force and moment sensors and two inertial sensors, were used (Fig. 4.3). On one shoe we mounted the transmitting transducer, on the other shoe the receiving transducer. Both transducers were placed in the sole of the forefoot in front of the inertial sensor (Fig. 4.4). The microcontroller and Bluetooth module were placed at the waist of the subject.

Six walking trials of two healthy volunteers were recorded.

\subsubsection{Reference measurement}

For a reference measurement, three reflective markers were placed at each forefoot as in Fig. 4.4. Their trajectories were recorded using six Vicon ${ }^{\circledR}$ cameras. From the three markers, orientation matrices - describing the orientation of right $\left(\boldsymbol{R}^{g r}\right)$ and left $\left(\boldsymbol{R}^{g l}\right)$ forefoot with respect to the Vicon-global frame $\left(\psi_{g}\right)$ - were constructed using cross products of the vectors connecting these markers. This was necessary to transform the vectors pointing from the toe markers to the ultrasound transducers $\left(\boldsymbol{p}_{u s, r}^{r}\right.$ and $\boldsymbol{p}_{u s, l}^{l}$, in right and left shoe frame, $\psi_{r}$ and $\psi_{l}$ respectively) to frame $\psi_{g}$ [35]. The reference distance measurement, $d_{\text {ref }}$, was then calculated

$$
d_{r e f}=\left\|\boldsymbol{p}_{\text {rtoe }}^{g}+\boldsymbol{R}^{g r} \boldsymbol{p}_{u s, r}^{r}-\left(\boldsymbol{p}_{\text {ltoe }}^{g}+\boldsymbol{R}^{g l} \boldsymbol{p}_{u s, l}^{l}\right)\right\|_{2} .
$$

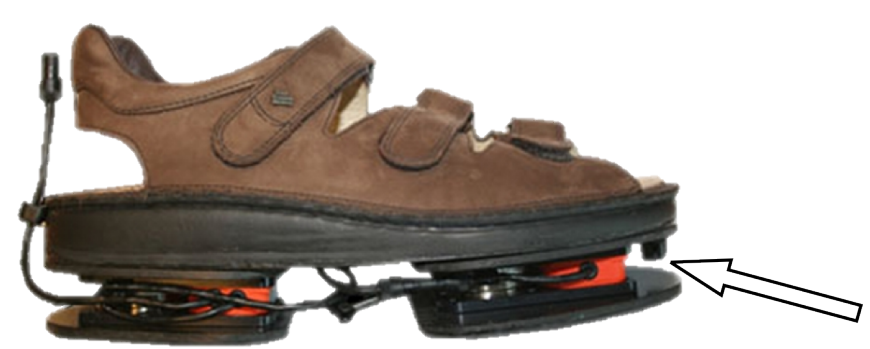

Figure 4.3: Xsens ForceShoe (Xsens Technologies B.V. 93]), containing two $6 \mathrm{D}$ force/moment sensors and two inertial sensors. The ultrasound transducers were mounted near the inertial sensor in the forefoot, indicated with the arrow. 


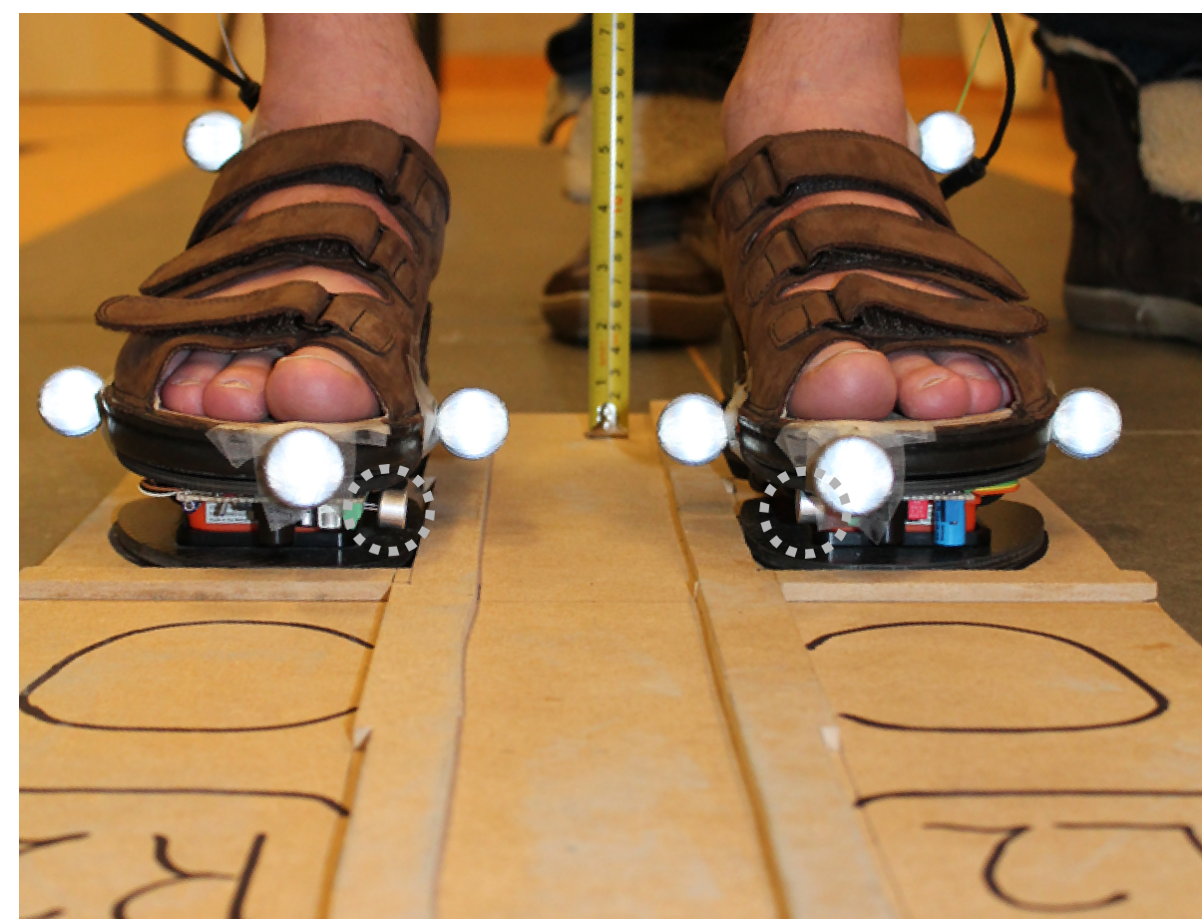

Figure 4.4: Measurement set-up for validating the ultrasound range measurements. The subject wearing the Xsens ForceShoes is standing on the calibration board. The ultrasound transducers were placed in the sole next to the inertial sensor in the front part (forefoot) of the ForceShoe, indicated with the dashed grey circles. For the reference measurement three reflective markers were placed on the forefoot segment of each ForceShoe. The ankle-markers were not used in this study.

Subsequently this distance was compared to the distance estimated with the ultrasound sensor system $\left(d_{\text {ultrasound }}\right)$.

\subsubsection{Calibration}

A calibration-board (Fig. 4.5) was developed to calibrate for speed of sound, which is temperature dependent 4.2, and for inaccuracies caused by the threshold method used for estimating the time of flight. On the board, each foot was placed on three different locations, similar to standing normally (e.g. L1R1) and walking with different step lengths (e.g. L1-R2). From these positions the distance between the ultrasound transducers was measured using a tape measure $\left(d_{\text {ruler }}\right)$. Based on the RMS of the ultrasound signal, two calibration regions were defined with two different offsets and gains. One region where the signal was not clipping, for RMS values below a threshold, and a region where the RMS exceeded that threshold and the amplified signal was clipping, as described in Section 4.2.1. For each set of measurements (in this case two) new 


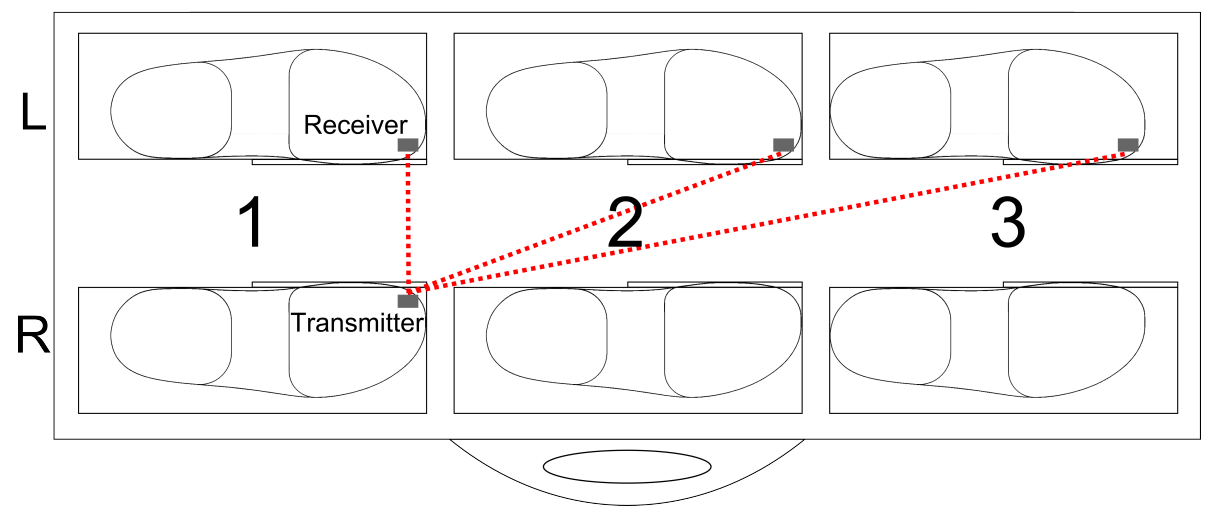

Figure 4.5: Calibration board used for calibrating the distance estimates. The distance between the transmitter and receiver was measured with a tape measure, after which calibration parameters were calculated. On the board, each foot can be placed on three different locations, similar to standing normally (e.g. R1-L1) and walking with different step lengths (e.g. R1-L2 or R1-L3). During al these combinations there is line of sight between transmitter and receiver, as indicated by the red dashed lines.

calibration measurements were performed, to calibrate the distance estimates (a constant temperature was assumed during a set of measurements).

\subsubsection{Synchronizing reference measurement and ultra- sound sensor}

To be able to compare both range estimates, it is important that they are synchronized in time. For this purpose, the cross correlation was calculated. The time for which the correlation was maximal was used to synchronize both ranges. Subsequently, the reference range estimates were resampled to match the range estimates from the ultrasound sensor, after which they were subtracted and the absolute value was taken,

$$
d_{a b s, \text { diff }}=\left|d_{\text {ref }}-d_{\text {ultrasound }}\right| .
$$

\subsection{Validation results}

\subsubsection{Calibration measurements}

The mean and standard deviations of the calibration measurements are listed in Table 4.1. Because the sensor system is not symmetric (one shoe contains a transmitter, the other a receiver) and because of inaccuracy of measuring the distance with the tape measure, calibration parameters are obtained from a combination of these measurements, causing the difference between $d_{\text {ruler }}$ and $d_{\text {ultrasound }}$. As can be seen in Table 4.1. the standard deviation from the 
Table 4.1: Overview of calibration measurements. $d_{\text {ruler }}$ is the distance measured with a tape measure. From the estimated distance with the ultrasound sensor, $d_{\text {ultrasound }}$, the mean and standard deviation (sd) are shown. Results are from one measurement, for each calibration position about 20 seconds.

\begin{tabular}{cccc}
\hline Calibration & $d_{\text {ruler }}(\mathrm{mm})$ & \multicolumn{2}{c}{$d_{\text {ultrasound }}(\mathrm{mm})$} \\
\cline { 3 - 4 } & & mean & sd \\
\hline R1-L1 & 137 & 137.0 & 0.1 \\
R1-L2 & 373 & 375.1 & 1.2 \\
R1-L3 & 683 & 680.3 & 2.5 \\
R3-L3 & 137 & 137.6 & 0.1 \\
R3-L2 & 351 & 349.1 & 0.9 \\
R3-L1 & 688 & 690.6 & 4.6 \\
\hline
\end{tabular}

R3-L1 measurements is relatively large. Therefore we calculate the signal-tonoise ratio (SNR) by estimating the mean power from two frequency regions; $40 \pm 3 \mathrm{kHz}$ and the remaining frequencies. The mean SNR for the R1-L1 measurement is 80 and for the R3-L1 it is 19. The relation between the SNR and the distance estimates is investigated in more detail in the next Section.

\subsubsection{Walking trials}

The absolute distance difference versus the SNR from all six walking trials is plotted in Fig.4.6. As expected, the SNR is small when the absolute distance difference $\left(d_{a b s, d i f f}\right)$ is large. This allows us to use the SNR as a measure for accepting or rejecting the ultrasound range estimates. For example, rejecting all estimates with an SNR less or equal to 5 shifts the mean of $d_{a b s, d i f f}$ from 110.7 to $7.0 \mathrm{~mm}$ (standard deviation from 203.7 to 7.1 ). When choosing the SNR threshold 20, the mean absolute difference is $5.8 \mathrm{~mm}$ with a standard deviation of 5.0. It should be noted that $52 \%$ of the distance estimates are rejected in this case, see Fig. 4.7

The comparison of the ultrasonic range estimates with the reference measurements for one walking trial is shown in Fig. 4.8. The estimates when setting the SNR threshold to 5 are also shown, rejecting $35 \%$ of the range estimates from all trials. The mean absolute difference for this trial is $6.7 \mathrm{~mm}$ (sd 5.3). For this SNR threshold, the distance differences over all trials are plotted versus the reference distance in Fig. 4.9 .

\subsection{Discussion}

For SNR thresholds larger than 20, the mean of the absolute distance difference stays between 5 and $6 \mathrm{~mm}$ (Fig. 4.7). SNR thresholds below 5 result in a mean absolute difference of $11.2(\mathrm{sd} 39.3) \mathrm{mm}$ or more. Therefore, it is recommended 


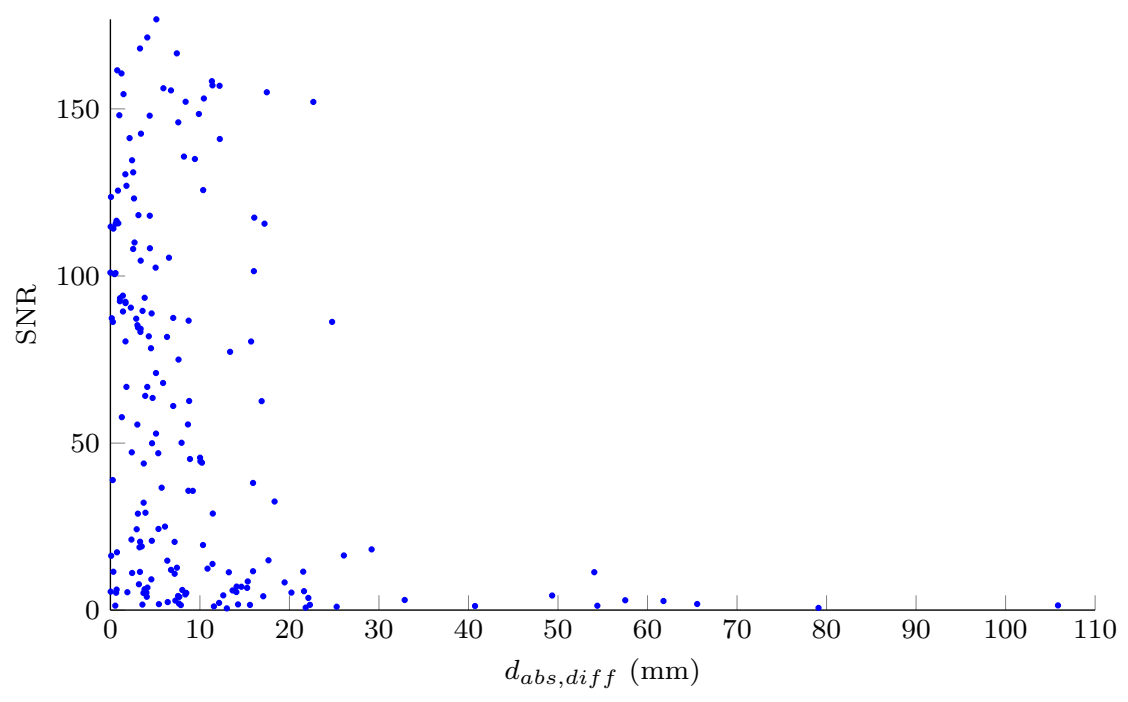

Figure 4.6: The absolute distance difference $\left(d_{a b s, \text { diff }}\right)$ versus the SNR from all six walking trials. The $x$-axis is limited for displaying purposes. Absolute differences of up to approximately $700 \mathrm{~mm}$ were observed, however, only for SNR values smaller than 5 .

to set the SNR threshold between 5 and 20. When setting the threshold to 20, $52 \%$ of the range estimates are rejected. This leaves approximately 7 distance estimates per second on average, which can be very useful for example for position estimation in a fusion algorithm together with inertial sensors with a higher sample rate. This sensor fusion is currently being investigated using methods from 22. In applications where more range estimates are required, the SNR threshold should be set lower.

When the distance between the transducers increases, the SNR becomes smaller (Section 4.4.1). When looking at the maximum distance that is measured by the sensor over all walking trials (Fig. 4.7. bottom), we see that when setting the SNR threshold to 8 or larger, distances larger than $\pm 700 \mathrm{~mm}$ are no longer detected. If this is important for the application, the SNR threshold should be set smaller than 8 .

It should be noted that the presented validation is a comparison with an optical system. Next to the errors of the positions of the optical system itself - which can be up to several millimeters [16 - there are errors introduced in measuring the distances between the transducers on the calibration board and the positions of the markers on the shoes with respect to the transducers. The calibration parameters obtained for example using L1-R2 and L1-R3 are different from the ones obtained using L3-R2 and L3-R1. This is why calibration parameters were obtained from a combination of all these measurements, which can cause errors. Once information about the walking cycle is available (that is, which foot is in front of the other), the calibration parameters correspond- 

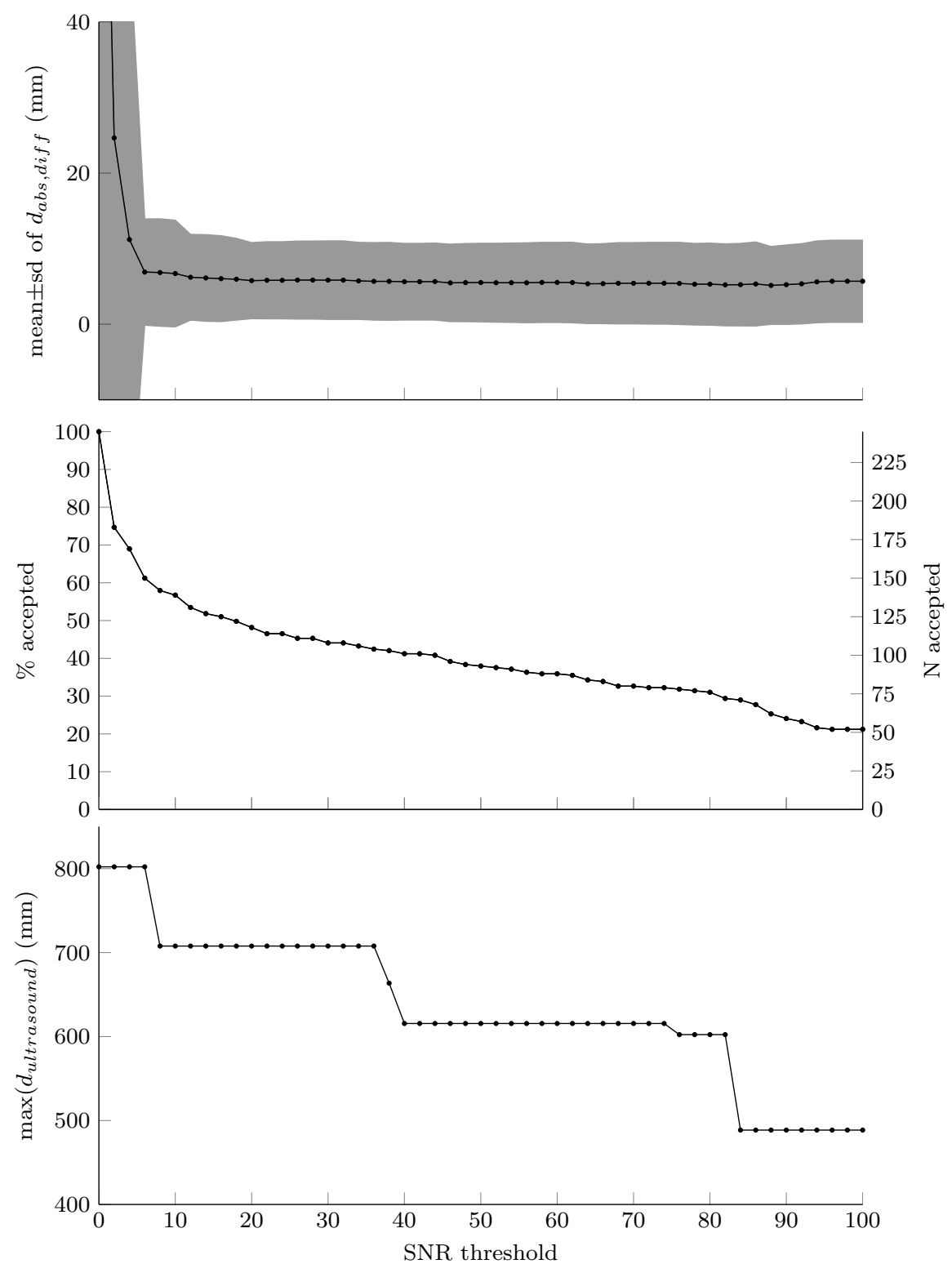

Figure 4.7: Top: the mean ( \pm sd, shaded) of $d_{a b s, \text { diff }}$ as a function of the SNR threshold for all walking trials (245 distance estimates). For display purposes the $y$ axis is limited; when no SNR threshold is set (SNR threshold=0), the mean absolute difference is 110.7 (sd 203.7) mm. Middle: the percentage (number (N) on right $y$ axis) of the estimates that are accepted for each value of the SNR threshold. Bottom: the maximum distance that is estimated during the walking trials, for each threshold. It can be seen that for SNR threshold larger than 40, distances larger than $\pm 600 \mathrm{~mm}$ are no longer detected. 

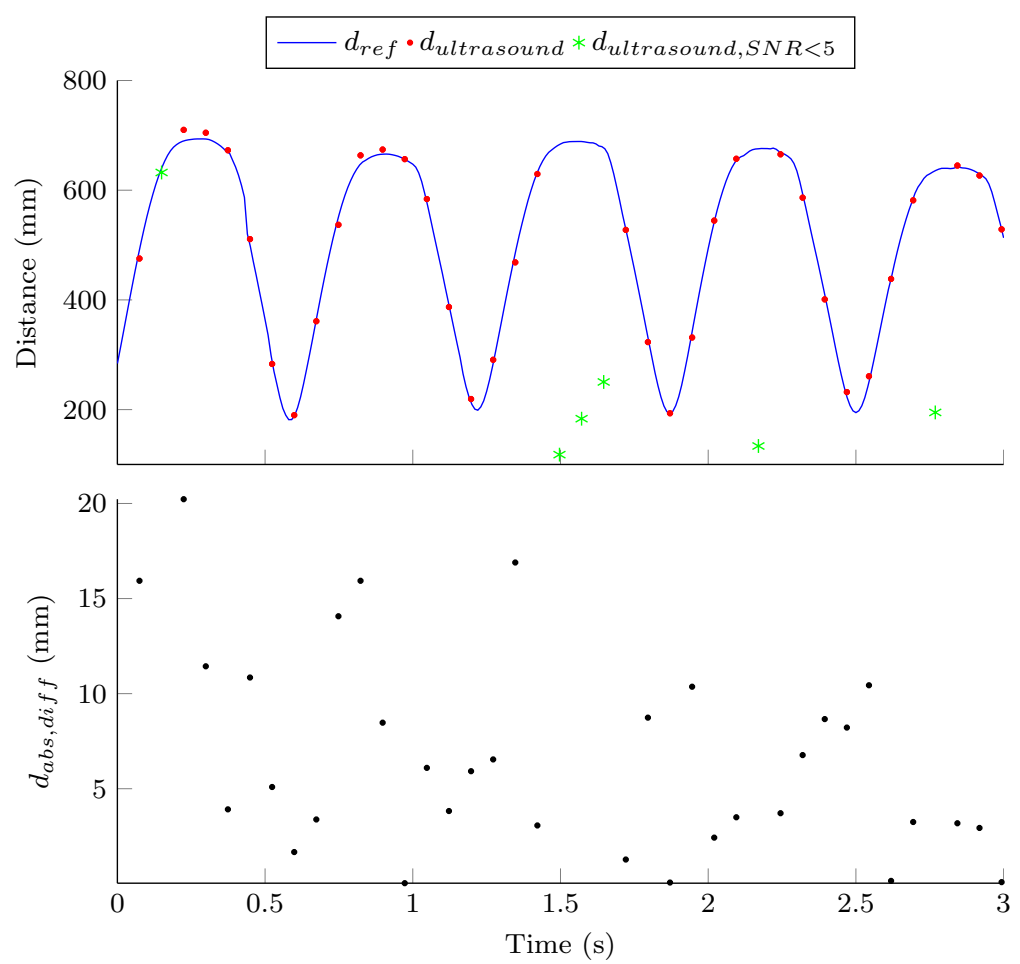

Figure 4.8: The reference distance $\left(d_{r e f}\right)$ and the distances estimated with the ultrasound sensor $\left(d_{\text {ultrasound }}\right)$ for one walking trial (top). Setting the SNR threshold to 5 results in the red estimates (the green estimates are rejected). The absolute difference is shown in the bottom figure (mean 6.7 , sd $5.3 \mathrm{~mm}$ ).

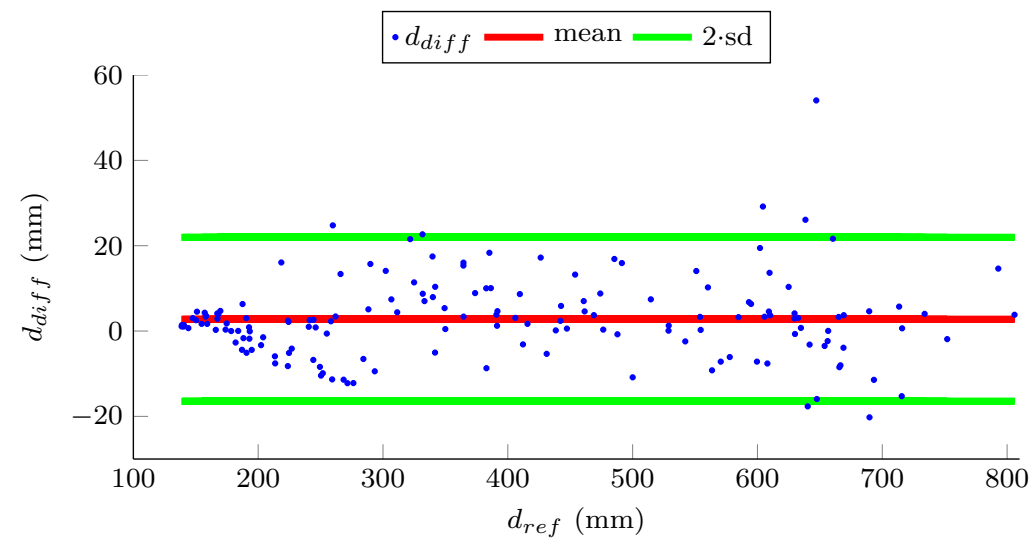

Figure 4.9: The difference between the distance estimated with the ultrasound sensor and the reference distance $\left(d_{\text {diff }}\right)$ versus the reference distance $\left(d_{r e f}\right)$ for all walking trials when using an SNR threshold of 5 . The mean $(2.6 \mathrm{~mm})$ and two times the standard deviation ( $\mathrm{sd}, 9.6 \mathrm{~mm}$ ) are indicated by the horizontal lines. 


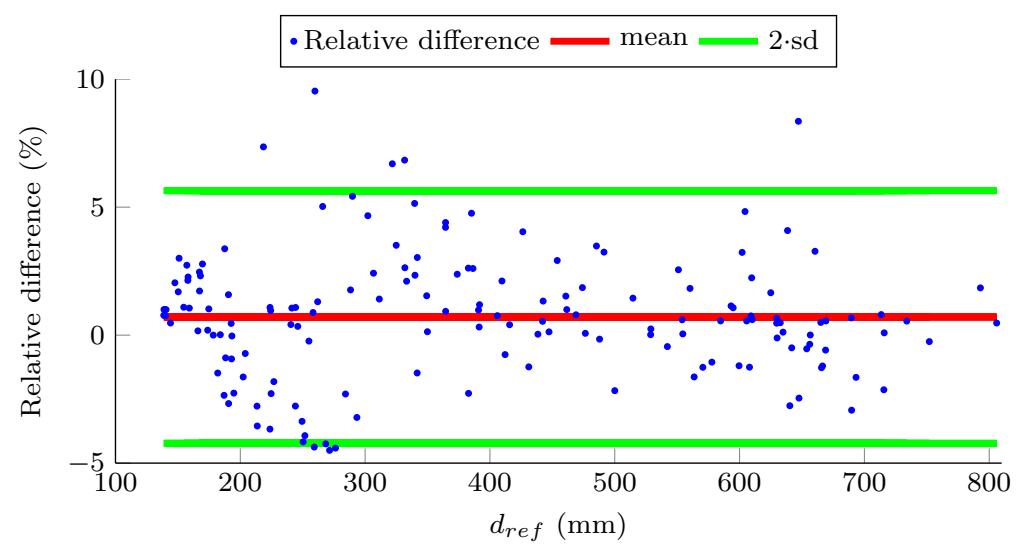

Figure 4.10: The relative difference (in \%) versus the reference distance $\left(d_{\text {ref }}\right)$ for all walking trials when using an SNR threshold of 5. The mean (0.7) and two times the standard deviation (sd, 2.5) are indicated by the horizontal lines.

ing to this can be used, to improve the accuracy. This information is available when adding information from inertial sensors.

When looking at the distance estimates in Fig. 4.8, we see approximately 5 steps of the walking trial, which is the limit of the optical reference system. From the figure the distance between the feet during double stance can be seen when looking at the maxima. The minima indicate the distance between the feet during midstance or midswing, i.e. the swinging foot passes the stance foot [8]. Alternating times between peaks or alternating heights of the peaks can be an indication of an asymmetric walking pattern.

In this study the ultrasound sensor was validated for measuring foot distance during gait. Our expectation is that, considering reflections from the floor, the performance of the sensor when used on other parts of the human body - for example, on trunk and hand for estimating maximum reach - will be similar. There should however be a direct line of sight between transmitter and receiver.

We validated the sensor using the ForceShoes of which the influence on gait appeared to be small [66]. The sensor can however be used of course on other shoes as well.

An offset is observed in the difference between the distance estimates, see Fig 4.9. This can be explained because the time of flight is more often underestimated than overestimated, as can also be seen in Fig. 4.8 (top). In Fig. 4.9 we also see that the distance difference is relatively small when feet are close together. For example, for reference distances smaller than $200 \mathrm{~mm}$ the mean absolute distance difference is $2.5 \mathrm{~mm}$ (sd $1.7 \mathrm{~mm}$ ) over all walking trials. In Fig. 4.10 the relative difference versus the reference distance is shown.

No significant correlation between velocity estimated with the reference system and $d_{a b s, \text { diff }}$ was found.

Disadvantage of the presented sensor is the amplifier, which is clipping when 
the feet are close together, causing the slope of the envelope to be steeper than normal. This problem is however solved by introducing two calibration regions; when the received signal is or is not clipping, estimated using the RMS of the received signal. Another solution could be to use a different amplifier, for example a time-gain-compensation amplifier which amplifies more when the time of flight becomes larger [95]. Another disadvantage are the required cables from microcontroller to both transducers, causing the microcontroller and Bluetooth module to be placed at the waist of the subject. This was necessary for time synchronization, that is, the microcontroller starts recording at the moment a pulse was sent to the transmitter. This could however be implemented wireless, given a suitable protocol (accuracy of maximally a few microseconds is needed [40] to keep the error below $1 \mathrm{~mm}$ ) is used. For the current calibration method it is assumed that the temperature stays constant during a set of measurements. If the temperature would change however during measurements - for example, when walking out of a heated room - this would immediately lead to an error in the estimated distance. A rule of thumb is that for a $10{ }^{\circ} \mathrm{C}$ change in temperature, the speed of sound and hence the estimated distance, changes with approximately $2 \%$ (see 4.2 and [57]). To overcome this problem a temperature sensor could be added to the system, provided that the body temperature is not influencing this sensor.

The time of flight is currently estimated using MATLAB ${ }^{\circledR}$. It is also possible to do this on a microcontroller and sending only the range estimates (and corresponding SNR) to the PC.

\subsection{Conclusion and future work}

An ultrasound sensor for estimating ranges on the human body is presented and validated using an optical reference system during gait. The SNR can be used as a measure for the uncertainty of a distance estimate. When rejecting all distance estimates with an SNR smaller than 5, the mean absolute distance difference between ultrasound sensor and reference system is $7.0 \mathrm{~mm}(\mathrm{sd} 7.1 \mathrm{~mm})$ over six walking trials. In this case $35 \%$ of the estimates are rejected.

In future work (Chapter 5) we will fuse the range measurements with inertial sensors for $3 \mathrm{D}$ estimation of (relative) positions of the feet. 
4. Ultrasonic range measurements on the human body 


\section{Chapter 5}

\section{Ambulatory estimation of relative foot positions by fusing ultrasound and inertial sensor data}

口

Published as:

D. Weenk, D. Roetenberg, B. J. F. van Beijnum, H. J. Hermens, P. H. Veltink Ambulatory estimation of relative foot positions by fusing ultrasound and inertial sensor data IEEE transactions on neural systems and rehabilitation engineering, vol.23, no.5, pp.817-826, Sept. 2015 http://dx.doi.org/10.1109/TNSRE.2014.2357686 


\section{Abstract}

Relative foot position estimation is important for rehabilitation, sports training and functional diagnostics. In this chapter an extended Kalman filter fusing ultrasound range estimates and inertial sensors is described. With this filter several gait parameters can be estimated ambulatory. Step lengths and stride widths from 54 walking trials of three healthy subjects were estimated and compared to an optical reference. Mean ( \pm standard deviation) of absolute difference was $1.7 \mathrm{~cm}( \pm 1.8 \mathrm{~cm})$ and $1.2 \mathrm{~cm}( \pm 1.2 \mathrm{~cm})$ for step length and stride width respectively. Walking with a turn and walking around in a square area were also investigated and resulted in mean absolute differences of $1.7 \mathrm{~cm}$ $( \pm 2.0 \mathrm{~cm})$ and $1.5 \mathrm{~cm}( \pm 1.5 \mathrm{~cm})$ for step lengths and stride widths. In addition to these relative positions, velocities, orientations and stance and swing times can also be estimated. We conclude that the presented system is low-cost and provides a complete description of footstep kinematics and timing. 


\subsection{Introduction}

A MBULATORY estimation of human gait is becoming increasingly pop1 ular in various applications such as rehabilitation, sports training and functional diagnostics [73. Advantages of ambulatory systems are that they can be used outside a laboratory and they are often less expensive than optical systems that require multiple cameras. Examples of wearable sensors are inertial sensors, flexible goniometers, electromagnetic tracking systems, sensing fabric, force sensors and electromyography [73.

An example of a wearable system that can be used for gait analysis is a shoe, instrumented with force or pressure sensors [67, 42, 63]. The center of mass can be estimated from force sensors under the foot and movement can be estimated using additional inertial sensors [69. The position of the projection of the center of mass on the ground with respect to the positions of the feet is important for balance [69, 70]. The relative positions of the feet are not measured directly with these shoes, but estimated from double integration of feet accelerations. However, this method leads to large position errors (drift) even when small errors in accelerations are measured.

Furthermore, only position changes are calculated and since the begin positions are unknown this does not result in relative positions of the feet. Stride lengths and lateral stride variability can however be estimated, as described for example by Rebula et al. in [56] and Schepers et al. in [70].

A method for estimating relative positions is to account for segment orientations and segment lengths 62. Disadvantage, however is that this leads to errors when segment lengths or orientations are incorrectly measured or estimated. Moreover, additional sensors (on the legs and pelvis) are needed.

A portable magnetic position and orientation tracker was presented by Roetenberg et al. [59. By using a 3D magnetic source, positioned on the back of the body, and 3D magnetic sensors placed at different body segments, the accuracy was approximately $8 \mathrm{~mm}$ in position and $5^{\circ}$ in orientation during movement. Disadvantage is the relatively large size and weight $(21 \mathrm{~cm}$ diameter, $11 \mathrm{~cm}$ height, $450 \mathrm{~g}$ ), making it unsuitable for placing it on a foot.

Regarding the use of ultrasound, Huitema et al. [30] described a motion analysis system, capable of measuring important human gait parameters. Two small ultrasonic receivers were attached to both shoes, while a transmitter was placed stationary on the floor. By subtracting the positions of the feet with zero velocity, step and stride lengths were estimated. Disadvantage is the transmitter placed on the floor, limiting the maximum measurement distance to $8.6 \mathrm{~m}$.

Vlasic et al. 80] presented a motion capture system by combining miniature inertial sensors with ultrasonic time of flight measurements. Orientations of several body segments were estimated and compared to an optical reference. Due to the range estimates, drift in orientations was reduced. Positions were not investigated in this study.

Zhao and Wang [97] also compensated the drift of inertial sensors with the 
assistance of magnetometers and an ultrasound emitter mounted on a moving vehicle and three receivers fixed on the ground. Although it was shown that drift in estimated positions was eliminated, the system is not completely ambulatory since the ultrasound receivers are fixed to the ground, similar to the study of Huitema et al. as described above.

More recently Hung and Suh [31] combined inertial sensors on both feet with a camera installed on one foot and infrared LEDs on the other, to measure relative position and orientation between the feet. They validated their system using marker pens attached to the shoes, which marked dots on white paper. The mean position error was $1.2-2.5 \mathrm{~cm}$, however the time in which vision data are available is small since the camera on the front of the right shoe needs to see the LEDs on the back of the left shoe. This makes it difficult to measure movements other than continuous walking along a straight line.

In this chapter the ambulatory estimation of the relative positions of the feet is presented using a minimal sensor configuration. An inertial sensor and an ultrasound transducer on each foot is shown to be enough for accurate estimation of gait parameters as step lengths and stride widths. Also velocities, orientations and stance and swing times are estimated. Advantages of the presented system are the low cost and the small size and weight. The required instrumentation is limited to the shoes of the subject. Furthermore, the system can be used everywhere and on every shoe, making it suitable for monitoring daily-live activities.

\subsection{Sensor fusion method}

In order to estimate the relative positions of the feet, the information from different sensors needs to be combined in an optimal way. Therefore, an extended Kalman filter (EKF) was designed. This Section describes the implementation of this filter after introducing the different sensors that are used.

\subsubsection{Sensor signals and models}

From the Xsens Forceshoe ${ }^{\mathrm{TM}}$ (Xsens B.V., Enschede, the Netherlands), the two inertial sensors in the forefeet segments were used. In each shoe an ultrasound transducer was mounted near the inertial sensor. A schematic drawing of the sensors and a definition of the coordinate frames is shown in Fig. 5.1. The global coordinate frame $\psi_{g}$ is defined with $x$-axis in walking direction, $y$-axis left and $z$-axis pointing up. The right shoe frame $\psi_{s, r}$ and the left shoe frame $\psi_{s, l}$ have the $x$-axis pointing to the front of the shoe, $y$-axis left and $z$-axis pointing up. The shoe frames are often shortened as $\psi_{s}$, when the same method applies to both shoes. 


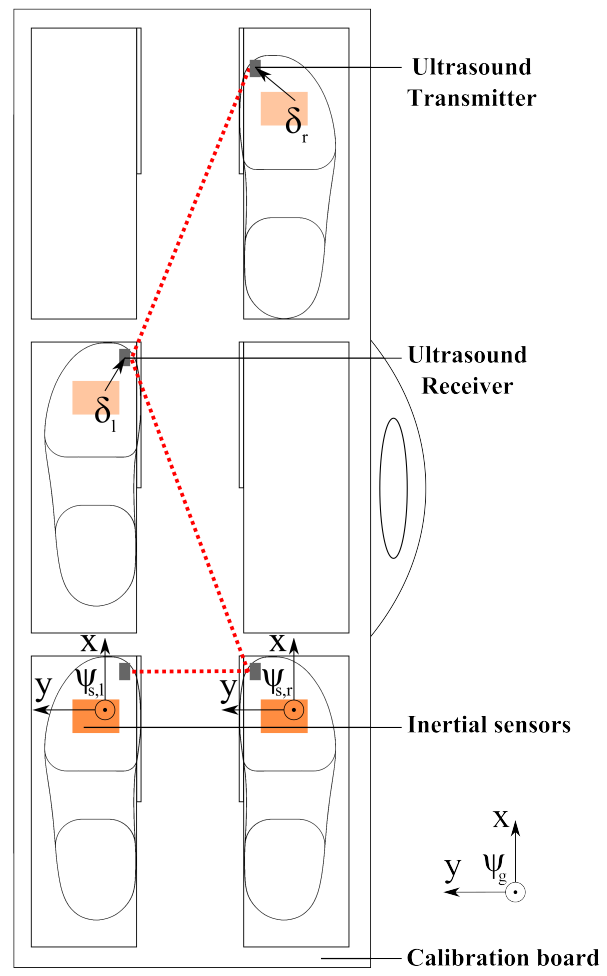

Figure 5.1: Schematic drawing of shoes on different positions on the ultrasound calibration board. Each shoe contains an inertial sensor and an ultrasound transducer. Dashed lines indicate that there is line of sight between both ultrasound transducers during walking. $\psi_{g}$ indicates the global coordinate frame, of which the $x$-axis points in the walking direction and the $z$-axis points up. $\psi_{s, l}$ and $\psi_{s, r}$ indicate the shoe coordinate frames of which the $x$-axis points forward, $z$-axis up. The shoe frames are often shortened as $\psi_{s}$, when the same method applies to both shoes. The vectors from accelerometer to transducer are indicated with $\delta_{r}$ and $\delta_{l}$ for right and left shoe respectively.

Symbols are indicated to be expressed in shoe or global coordinate frame by using the superscripts $s$ and $g$ respectively. The orientation of a foot is indicated as $\boldsymbol{R}^{g s, r}$ (and $\boldsymbol{R}^{g s, l}$ ), meaning the orientation of frame $\psi_{s, r}$ (and frame $\left.\psi_{s, l}\right)$ with respect to frame $\psi_{g}$. This is also shortened as $\boldsymbol{R}^{g s}$ when a method applies to both shoes.

A measured gyroscope signal $\boldsymbol{y}_{G}$ is described as the sum of angular velocity $\boldsymbol{\omega}^{s}$, a slowly varying offset (or bias) $\boldsymbol{b}^{s}$ - for example, caused by changes in temperature - and Gaussian noise $\boldsymbol{e}_{G}$, all in shoe coordinate frame $\psi_{s}$, 45, 61]

$$
\boldsymbol{y}_{G}=\boldsymbol{\omega}^{s}+\boldsymbol{b}^{s}+\boldsymbol{e}_{G}
$$

For readability the superscript $s$ is omitted in the remainder of this chapter for angular velocities and gyro bias, since they are all expressed in shoe coordinate 


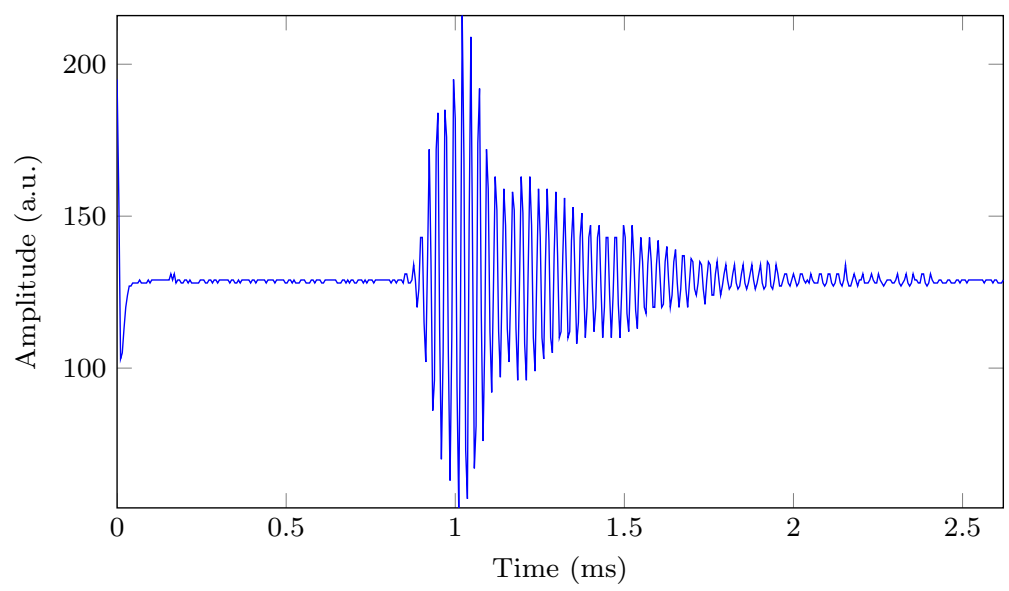

Figure 5.2: Typical example of a recorded ultrasound signal. The signal is bandpass filtered and normalized after which the envelope is calculated. From the envelope the time of flight is estimated using a threshold method.

frame.

A measured accelerometer signal $\boldsymbol{y}_{A}$ is described as the sum of sensor acceleration $\boldsymbol{a}^{s}$, gravitational acceleration $\boldsymbol{g}^{s}$ and Gaussian noise $\boldsymbol{e}_{A}$, all in shoe coordinate frame $\psi_{s}$

$$
\boldsymbol{y}_{A}=\boldsymbol{a}^{s}-\boldsymbol{g}^{s}+\boldsymbol{e}_{A}
$$

Accelerations and angular velocities were recorded at $50 \mathrm{~Hz}$.

The ultrasound range estimation is described and validated in the previous chapter [86], a summary is given here. With two ultrasound transducers (Prowave 400ST/R120), mounted in the forefeet of the ForceShoes ${ }^{\mathrm{TM}}$, the range between the feet is estimated. A microcontroller is used to send a pulse to the transmit transducer. This pulse causes the transducer to output a $40 \mathrm{Khz}$ pulse. At the same time the pulse is sent, the microcontroller starts to record the received signal (Fig. 5.2), which is then band-pass filtered to eliminate noise. After envelope detection a threshold method is used to estimate the time of flight $\left(t_{T o F}\right)$. After multiplying with speed of sound $\left(v_{s}\right)$, this gives a first estimate of the distance $\left(d_{U}\right)$ between the transducers

$$
d_{U}=v_{s} \cdot t_{T o F}
$$

The speed of sound is temperature dependent and in air the expression is

$$
v_{s}=331.4 \cdot\left(1+1.83 \cdot 10^{-3} \cdot T_{C}\right),
$$

with $T_{C}$ the temperature in ${ }^{\circ} \mathrm{C}$ [57]. Range estimates are calibrated using a calibration board (Fig. 5.1 at which the feet can be placed at different (known) locations. It is assumed that the temperature is constant during a 
set of measurements. The signal to noise ratio (SNR) is estimated and used as a measure for the uncertainty of each range estimate. The distances and corresponding SNR are estimated approximately 13.3 times per second. The SNR can also be used to reject distance estimates. In general this will increase accuracy, but decrease the effective update rate [86].

\subsubsection{Filter structure and notations}

The proposed filter is an extended Kalman filter (EKF), with the goal to estimate a state vector $\boldsymbol{x}$ and its covariance $\boldsymbol{P}$. The state vector contains 24 states. For right and left foot (indicated with subscripts $r$ and $l$ ) the 3D position $\boldsymbol{p}$ and velocity $\boldsymbol{v}$ are estimated. Furthermore, to express accelerations in a global coordinate frame and for an accurate subtraction of gravitational accelerations, the orientation of each foot $\boldsymbol{R}^{g s}$ must be estimated. In [61, it has been proven to be useful for orientation estimation to use orientation error and gyroscope bias error, describing the difference between true and estimated values. This way, inertial sensor relations can be described as linear processes when the error is assumed to be small. Therefore, orientation error $\boldsymbol{\theta}_{\epsilon}$ and gyroscope bias error $\boldsymbol{b}_{\epsilon}$ are also included in the state vector, which then becomes

$$
\boldsymbol{x}=\left(\begin{array}{llllllll}
\boldsymbol{p}_{r} & \boldsymbol{p}_{l} & \boldsymbol{v}_{r} & \boldsymbol{v}_{l} & \boldsymbol{\theta}_{\epsilon, r} & \boldsymbol{\theta}_{\epsilon, l} & \boldsymbol{b}_{\epsilon, r} & \boldsymbol{b}_{\epsilon, l}
\end{array}\right)^{T}
$$

The structure of the filter is shown Fig. 5.3. After an initialization, each filter iteration contains three steps. First, the states are predicted based on the sensor models. After this prediction step, the predicted states $\hat{\boldsymbol{x}}^{-}$are corrected during the measurement update. Finally, the estimated orientation $\hat{\boldsymbol{R}}^{g s}$ and gyroscope bias $\hat{\boldsymbol{b}}$ are updated according to the orientation error $\boldsymbol{\theta}_{\epsilon}$ and gyroscope bias error $\boldsymbol{b}_{\epsilon}$ and the estimated orientation and bias from the previous step. These four blocks are described in the next Sections.

A hat on a variable indicates an estimate, a minus superscript indicates an a priori estimate; that is, before applying the measurement updates. The subscript $k$ indicates the filter time step.

\subsubsection{Initialization}

The initial $(k=0)$ orientation of the feet $\hat{\boldsymbol{R}}_{0}^{g s}\left(\psi_{s, r}\right.$ and $\psi_{s, l}$ with respect to $\left.\psi_{g}\right)$ is based on an initial inclination and an initial heading estimate. The initial inclination is estimated from the first $0.5 \mathrm{~s}$ of the accelerometer signal, assuming the subject is standing still and only gravitational acceleration is measured. The initial heading is estimated by running the complete filter once, without applying the ultrasound range updates. From the difference in estimated positions, the initial orientation of each inertial sensor is estimated. It is also possible to set the heading manually, with the feet parallel. The heading is then corrected automatically after a few steps. However, this would make the validation more difficult, since only a few steps are available in our measurements due to the limited optical measurement volume. 


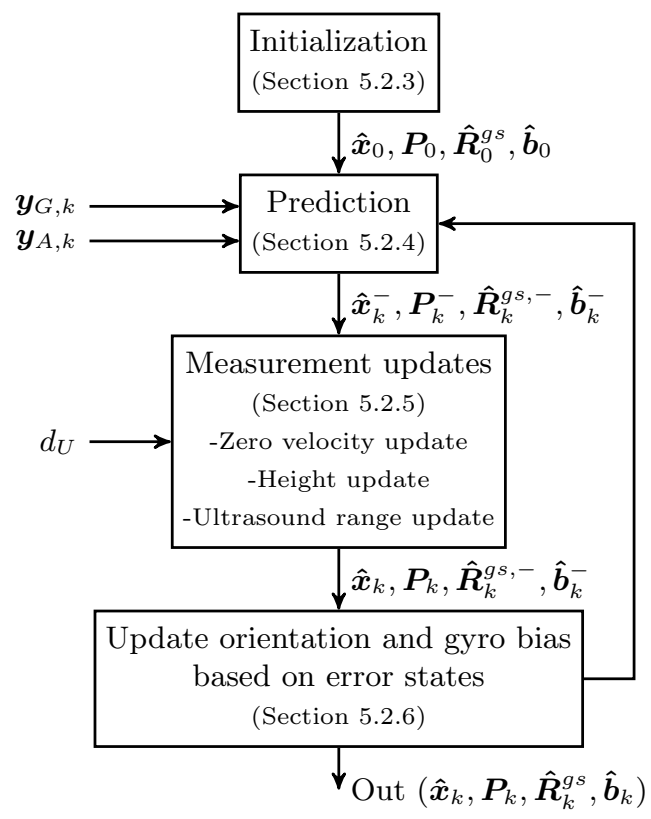

Figure 5.3: Filter structure for estimating the state vector $\boldsymbol{x}$ and corresponding covariance $\boldsymbol{P}$. After initialization $(k=0)$ the filter is started with a prediction step, followed by different measurement updates. Subsequently, the orientation $\hat{\boldsymbol{R}}^{g s}$ and gyroscope bias $\hat{\boldsymbol{b}}$ are updated based on the error states and the estimates from the previous time step $k-1$. Inputs are accelerometer and gyroscope signals $\boldsymbol{y}_{A}$ and $\boldsymbol{y}_{G}$ and ultrasound range $d_{U}$. The measurement updates from the ultrasonic sensor are applied at a different rate $(13.3 \mathrm{~Hz})$ than the predictions based on the inertial sensors $(50 \mathrm{~Hz})$.

The initial $x$ and $y$ positions of the right foot are set to zero, as is the initial $x$ position of the left foot. The $y$ position of the left foot is set to the range estimated with the ultrasound system. $z$ positions of both feet are set to the physical height of the accelerometer, measured with a ruler when the shoe was on a flat surface. The initial velocities and error states are all set to zero and the initial gyroscope bias $\hat{\boldsymbol{b}}_{0}$ is estimated by taking the mean of the gyroscope signal of the first $0.5 \mathrm{~s}$. Therefore it is useful that the subject is standing still with both feet flat on the floor at the beginning of each measurement.

\subsubsection{Prediction}

The goal of this step is to predict the states $\boldsymbol{x}$ and the corresponding uncertainty $\boldsymbol{P}$. The equations for this are described in this Section. For clarity the prediction for one foot is described, since it is identical for both feet. Consequently, the state vector $\boldsymbol{x}$ is simplified to (12 states)

$$
\boldsymbol{x}=\left(\begin{array}{llll}
\boldsymbol{p} & \boldsymbol{v} & \boldsymbol{\theta}_{\epsilon} & \boldsymbol{b}_{\epsilon}
\end{array}\right)^{T}
$$


In the subsequent Section 5.2.5 the states for left and right foot are linked during the measurement updates.

\section{Gyroscope bias and error orientation}

To be able to accurately estimate 3D positions, the orientation of the inertial sensors needs to be estimated in order to be able to subtract gravitational acceleration from accelerometer measurements, according to formula (5.2).

The gyroscope offset is modeled as a first order Markov process driven by white Gaussian noise $\boldsymbol{w}_{b, k}$ 45]

$$
\boldsymbol{b}_{k}=\boldsymbol{b}_{k-1}+\boldsymbol{w}_{b, k}
$$

Therefore, the prediction of the gyroscope bias $\hat{\boldsymbol{b}}_{k}^{-}$is

$$
\hat{\boldsymbol{b}}_{k}^{-}=\hat{\boldsymbol{b}}_{k-1}
$$

where the minus superscript indicates that it is an a priori estimate; that is, before applying the measurement updates. The gyroscope bias error $\boldsymbol{b}_{\epsilon}$ is defined as the difference between estimated and true gyroscope bias

$$
\boldsymbol{b}_{\epsilon, k}=\hat{\boldsymbol{b}}_{k}-\boldsymbol{b}_{k}
$$

If we substitute equations 5.7 and 5.8 in 5.9 we obtain

$$
\boldsymbol{b}_{\epsilon, k}=\boldsymbol{b}_{\epsilon, k-1}-\boldsymbol{w}_{b, k}
$$

For small orientation errors, a linear model relating estimated orientation $\hat{\boldsymbol{R}}^{g s}$, true orientation $\boldsymbol{R}^{g s}$ and orientation error $\boldsymbol{\theta}_{\epsilon}$ can be used [45, 68, 7]

$$
\hat{\boldsymbol{R}}^{g s} \approx \boldsymbol{R}^{g s}\left(\boldsymbol{I}+\left[\boldsymbol{\theta}_{\epsilon} \times\right]\right)
$$

in which $\left[\boldsymbol{\theta}_{\epsilon} \times\right]$ is a skew symmetric matrix, which is obtained by using the cross product operator on a vector 7 ]

$$
[\boldsymbol{a} \times]=\left(\begin{array}{ccc}
0 & -a_{z} & a_{y} \\
a_{z} & 0 & -a_{x} \\
-a_{y} & a_{x} & 0
\end{array}\right)
$$

To be able to predict the orientation error, the relation between previous and current orientation error state is needed. Therefore, the time derivative of $\left[\boldsymbol{\theta}_{\epsilon} \times\right]$ is calculated. After substituting the differential equation to integrate angular velocities to orientation, $\dot{\boldsymbol{R}}=\boldsymbol{R}[\boldsymbol{\omega} \times]$, this results in (see 68. for a detailed description)

$$
\dot{\boldsymbol{\theta}}_{\epsilon}=[\boldsymbol{\omega} \times] \boldsymbol{\theta}_{\epsilon}-\boldsymbol{b}_{\epsilon}
$$

after discretization using methods in 22] we get

$$
\begin{aligned}
\boldsymbol{\theta}_{\epsilon, k}= & \left(\boldsymbol{I}_{3}+T[\boldsymbol{\omega} \times]+\frac{T^{2}}{2}[\boldsymbol{\omega} \times]^{2}\right) \boldsymbol{\theta}_{\epsilon, k-1} \\
& +\left(-T \boldsymbol{I}_{3}-\frac{T^{2}}{2}[\boldsymbol{\omega} \times]\right) \boldsymbol{b}_{\epsilon, k-1}
\end{aligned}
$$




\section{Position and velocity}

For the velocity $\boldsymbol{v}^{g}$ and position $\boldsymbol{p}^{g}$ in global coordinate frame, we have

$$
\boldsymbol{v}_{k}^{g}=\boldsymbol{v}_{k-1}^{g}+T \hat{\boldsymbol{a}}_{k-1}^{g}
$$

and

$$
\boldsymbol{p}_{k}^{g}=\boldsymbol{p}_{k-1}^{g}+T \boldsymbol{v}_{k-1}^{g}+\frac{T^{2}}{2} \hat{\boldsymbol{a}}_{k-1}^{g}
$$

Therefore we need an estimate of the acceleration $\hat{\boldsymbol{a}}^{g}$ in global coordinate frame. After substituting the sensor model in equation (5.2) into equation (5.17a) and the relation between estimated orientation $\left(\hat{\boldsymbol{R}}^{g s}\right)$ and true orientation $\left(\boldsymbol{R}^{g s}\right)$ in equation (5.11) into equation (5.17b), this results in

$$
\begin{aligned}
\hat{\boldsymbol{a}}^{g} & =\boldsymbol{R}^{g s} \hat{\boldsymbol{a}}^{s} \\
& =\boldsymbol{R}^{g s}\left(\boldsymbol{y}_{A}+\boldsymbol{g}^{s}\right) \\
& =\hat{\boldsymbol{R}}^{g s} \boldsymbol{y}_{A}+\hat{\boldsymbol{R}}^{g s}\left[\boldsymbol{y}_{A} \times\right] \boldsymbol{\theta}_{\epsilon}+\boldsymbol{g}^{g}
\end{aligned}
$$

in which the subscript $k$ has been omitted for readability

In matrix form the prediction is now described as

$$
\hat{\boldsymbol{x}}_{k}^{-}=\boldsymbol{F} \hat{\boldsymbol{x}}_{k-1}+\boldsymbol{u}_{k-1}
$$

where $\boldsymbol{F}$ can be derived from $5.10,5.14,5.15$, and 5.16

$$
\begin{gathered}
\boldsymbol{F}= \\
\left(\begin{array}{cccc}
\boldsymbol{I}_{3} & T & \frac{T^{2}}{2} \hat{\boldsymbol{R}}^{g s}\left[\boldsymbol{y}_{A} \times\right] & \mathbf{0}_{3} \\
\mathbf{0}_{3} & \boldsymbol{I}_{3} & T \hat{\boldsymbol{R}}^{g s}\left[\boldsymbol{y}_{A} \times\right] & \mathbf{0}_{3} \\
\mathbf{0}_{3} & \mathbf{0}_{3} & \boldsymbol{I}_{3}+T[\boldsymbol{\omega} \times]+\frac{T^{2}}{2}[\boldsymbol{\omega} \times]^{2} & -T \boldsymbol{I}_{3}-\frac{T^{2}}{2}[\boldsymbol{\omega} \times] \\
\mathbf{0}_{3} & \mathbf{0}_{3} & \mathbf{0}_{3} & \boldsymbol{I}_{3}
\end{array}\right)
\end{gathered}
$$

The input vector $\boldsymbol{u}$ can be described as

$$
\boldsymbol{u}=\left(\begin{array}{c}
\frac{T^{2}}{2}\left(\hat{\boldsymbol{R}}^{g s} \boldsymbol{y}_{A}+\boldsymbol{g}\right) \\
T\left(\hat{\boldsymbol{R}}^{g s} \boldsymbol{y}_{A}+\boldsymbol{g}\right) \\
\mathbf{0}_{3 \times 1} \\
\mathbf{0}_{3 \times 1}
\end{array}\right)
$$

In both $\boldsymbol{F}$ and $\boldsymbol{u}$ the subscript $k$ has been omitted for readability, however, these matrices need to be updated each time step.

The covariance matrix $\boldsymbol{P}$, describing the uncertainty of the state vector $\boldsymbol{x}$, is updated using

$$
\boldsymbol{P}_{k}^{-}=\boldsymbol{F} \boldsymbol{P}_{k-1} \boldsymbol{F}^{T}+\boldsymbol{Q}
$$

with $\boldsymbol{Q}$ the process noise covariance matrix [22, 90]. After the prediction step the error states are set to zero. 


\subsubsection{Measurement updates}

Zero-velocity- and height updates of each foot are performed during stance. This is detected using the algorithm described by Skog et al. in [71. If a combination of accelerometer and gyroscope signals $\left(\boldsymbol{y}_{A}\right.$ and $\left.\boldsymbol{y}_{G}\right)$ is below a certain threshold, the algorithm decides that the inertial sensor is not moving. To avoid conflicts with height and zero velocity updates, the ultrasound range updates are also performed based on this stance detection. This was done by updating only the position of the moving foot (or last moved foot during double stance).

All measurement updates are performed using the following equations (see also [22, 90]) and are described individually below.

The Kalman gain is

$$
\boldsymbol{K}_{k}=\boldsymbol{P}_{k}^{-} \boldsymbol{H}^{T}\left(\boldsymbol{H} \boldsymbol{P}_{k}^{-} \boldsymbol{H}^{T}+\boldsymbol{R}\right)^{-1}
$$

with $\boldsymbol{R}$ the measurement noise covariance. The estimates $\hat{\boldsymbol{x}}_{k}^{-}$(predicted states) are updated based on the measurements $\boldsymbol{z}$ of the different sensors

$$
\hat{\boldsymbol{x}}_{k}=\hat{\boldsymbol{x}}_{k}^{-}+\boldsymbol{K}_{k}\left(\boldsymbol{z}_{k}-\boldsymbol{H} \hat{\boldsymbol{x}}_{k}^{-}\right)
$$

and the error covariance matrix $\boldsymbol{P}_{k}^{-}$is updated using

$$
\boldsymbol{P}_{k}=\left(\boldsymbol{I}-\boldsymbol{K}_{k} \boldsymbol{H}\right) \boldsymbol{P}_{k}^{-}
$$

\section{Zero velocity update}

During stance the velocity is updated to be zero using

$$
\boldsymbol{z}_{v 0}=\boldsymbol{H}_{v 0} \boldsymbol{x}+\boldsymbol{e}_{v 0}=\mathbf{0}_{3 \times 1}
$$

with

$$
\begin{aligned}
& \boldsymbol{H}_{v 0, r f}=\left(\begin{array}{cccc}
\mathbf{0}_{3 \times 6} & \boldsymbol{I}_{3} & \mathbf{0}_{3 \times 3} & \mathbf{0}_{3 \times 12}
\end{array}\right) \\
& \boldsymbol{H}_{v 0, l f}=\left(\begin{array}{llll}
\mathbf{0}_{3 \times 6} & \mathbf{0}_{3 \times 3} & \boldsymbol{I}_{3} & \mathbf{0}_{3 \times 12}
\end{array}\right)
\end{aligned}
$$

The white Gaussian measurement noise is indicated with $\boldsymbol{e}_{v 0}$, describing the uncertainty of this measurement update. Because the relation between acceleration and orientation error in (5.17), the zero velocity update also stabilizes inclination. This is because during stance, zero velocity is assumed (see description at the beginning of this Section) and the accelerometers measure pure gravitational acceleration. 


\section{Height update}

During stance the height is updated to be equal to the initial height, assuming walking on a flat floor.

$$
z_{h}=\boldsymbol{H}_{h} \boldsymbol{x}+e_{h}=p_{z, \text { init }}
$$

with

$$
\begin{aligned}
\boldsymbol{H}_{h, r} & =\left(\begin{array}{lllllll}
0 & 0 & 1 & 0 & 0 & 0 & \mathbf{0}_{1 \times 18}
\end{array}\right) \\
\boldsymbol{H}_{h, l} & =\left(\begin{array}{lllllll}
0 & 0 & 0 & 0 & 0 & 1 & \mathbf{0}_{1 \times 18}
\end{array}\right)
\end{aligned}
$$

The white Gaussian measurement noise is indicated with $e_{h}$, describing the uncertainty of this measurement update.

\section{Ultrasound range update}

The ultrasound range updates are performed during stance and swing, as described above. The update is described as the range between the ultrasound transducers, expressed in filter positions. With the filter, the $3 \mathrm{D}$ positions of the accelerometers on the right and left foot are estimated (part of the state vector in equation (5.5), that is

$$
\boldsymbol{p}_{r}=\left(\begin{array}{c}
p_{r x} \\
p_{r y} \\
p_{r z}
\end{array}\right), \quad \boldsymbol{p}_{l}=\left(\begin{array}{c}
p_{l x} \\
p_{l y} \\
p_{l z}
\end{array}\right)
$$

Therefore, for each foot an additional position vector $\boldsymbol{\delta}^{s}$, pointing from the accelerometer to the ultrasound transducer is needed (see Fig. 5.1). These vectors need to be expressed in global coordinate frame $\psi_{g}$ using the orientation $\boldsymbol{R}^{g s}$ of each foot

$$
\boldsymbol{R}^{g s, r} \boldsymbol{\delta}_{r}^{s, r}=\boldsymbol{\delta}_{r}^{g}=\left(\begin{array}{c}
\delta_{r x} \\
\delta_{r y} \\
\delta_{r z}
\end{array}\right), \quad \boldsymbol{R}^{g s, l} \boldsymbol{\delta}_{l}^{s, l}=\boldsymbol{\delta}_{l}^{g}=\left(\begin{array}{c}
\delta_{l x} \\
\delta_{l y} \\
\delta_{l z}
\end{array}\right)
$$

The ultrasound range update can now be described as the euclidean norm of the difference in position of both ultrasound transducers

$$
\begin{aligned}
z_{\text {range }} & =h(\boldsymbol{x})+e_{\text {range }} \\
& =\left\|\boldsymbol{p}_{r}+\boldsymbol{\delta}_{r}^{g}-\left(\boldsymbol{p}_{l}+\boldsymbol{\delta}_{l}^{g}\right)\right\|_{2}+e_{\text {range }}
\end{aligned}
$$

The white Gaussian measurement noise is indicated with $e_{\text {range }}$, describing the uncertainty of this measurement update. 
The range updates are split in two updates, one in which the right foot position $\boldsymbol{p}_{r}$ is updated and one in which the left foot position $\boldsymbol{p}_{l}$ is updated. The (last) moving foot is updated, based on the contact detection. The derivatives of equation (5.31) to the $3 \mathrm{D}$ components of the right and left foot positions are calculated

$$
\frac{d h}{d p_{r x}}=\frac{p_{r x}+\delta_{r x}-p_{l x}-\delta_{l x}}{\left\|\boldsymbol{p}_{r}+\boldsymbol{\delta}_{r}^{g}-\left(\boldsymbol{p}_{l}+\boldsymbol{\delta}_{l}^{g}\right)\right\|_{2}}
$$

with similar results for the derivatives to the other components.

Subsequently, these derivatives are used in the Jacobians of these two measurement updates

$$
\begin{aligned}
& \boldsymbol{H}_{\text {range }, r}=
\end{aligned}
$$

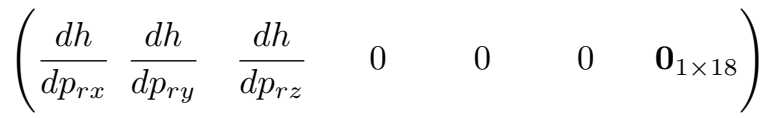

$$
\begin{aligned}
& \boldsymbol{H}_{\text {range }, l}=
\end{aligned}
$$

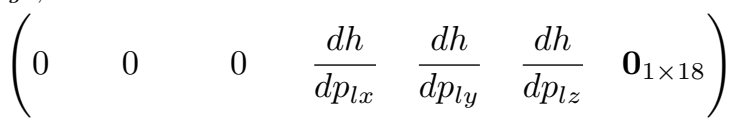

Outliers are detected and rejected when the normalized innovations squared (NIS) exceeded a threshold [22] [76].

$$
\begin{aligned}
& N I S_{k}= \\
& \quad\left(\boldsymbol{y}_{k}-\boldsymbol{H}_{k} \hat{\boldsymbol{x}}_{k}\right)^{T}\left(\boldsymbol{H}_{k}^{T} \boldsymbol{P}_{k} \boldsymbol{H}_{k}+\boldsymbol{R}_{k}\right)^{-1}\left(\boldsymbol{y}_{k}-\boldsymbol{H}_{k} \hat{\boldsymbol{x}}_{k}\right)
\end{aligned}
$$

\subsubsection{Update orientation and gyro bias}

In each iteration, the estimated orientation $\hat{\boldsymbol{R}}^{g s}$ and gyro bias $\hat{\boldsymbol{b}}$ are updated using the error states $\boldsymbol{\theta}_{\epsilon}$ and $\boldsymbol{b}_{\boldsymbol{\epsilon}}$ (based on (5.11)) using

$$
\begin{aligned}
\hat{\boldsymbol{R}}_{k}^{g s} & =\hat{\boldsymbol{R}}_{k}^{g s,-}\left(\boldsymbol{I}-\left[\boldsymbol{\theta}_{\epsilon, k} \times\right]\right) \\
\hat{\boldsymbol{b}}_{k} & =\hat{\boldsymbol{b}}_{k}^{-}+\boldsymbol{b}_{\epsilon, k}
\end{aligned}
$$

The rotation matrix $\hat{\boldsymbol{R}}_{k}^{g s}$ is subsequently orthogonalized using singular value decomposition 48 .

\subsection{Validation method}

\subsubsection{Set-up}

On one shoe we mounted an ultrasound transducer sending a $40 \mathrm{kHz}$ pulse to a similar transducer on the other shoe. Both transducers were placed in the sole of the forefoot in front of the inertial sensor (Fig. 5.4). For the validation of the 


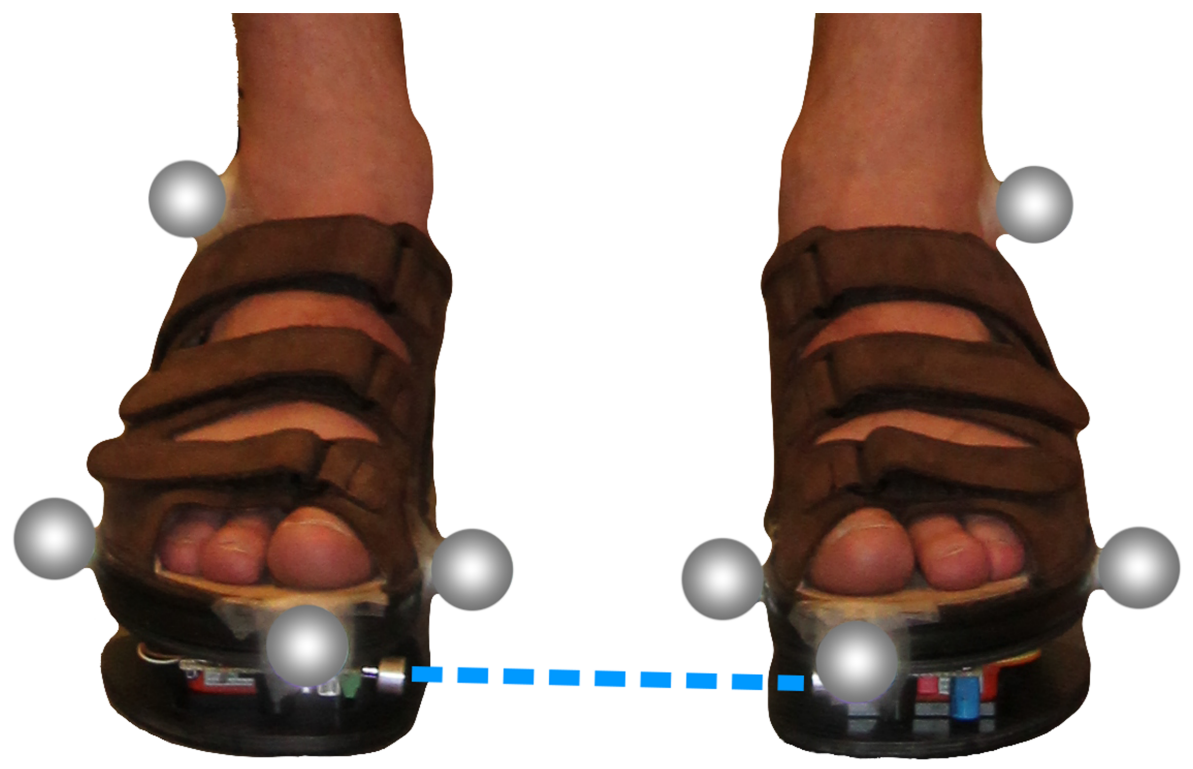

Figure 5.4: Measurement set-up for validating the ultrasound range measurements. The ultrasound transducers were placed in the sole next to the inertial sensor in the front part (forefoot) of the ForceShoe ${ }^{\mathrm{TM}}$. The range is indicated by the dashed line. Three reflective markers were placed on the forefoot segment of each ForceShoe ${ }^{\mathrm{TM}}$. The ankle markers were not used in this study.

presented algorithm, the positions of the feet were measured using six Vicon ${ }^{\circledR}$ cameras measuring the position of three reflective markers attached to each shoe (Fig. 5.4). 54 walking trials from three healthy subjects were recorded in which the subject walked in a straight line at their own comfortable walking speed. The range was limited due to the Vicon ${ }^{\circledR}$ measurement volume, hence subjects walked 6 steps (about $3.5 \mathrm{~m}$ ). Also trials were recorded were subjects walked 4 steps, to be certain to hit the force plates, which was needed for another study.

To demonstrate the robustness of our algorithm, two trials were recorded in which the subject walked around in the (square) measurement volume of the Vicon ${ }^{\circledR}$ system (about 14 steps with each foot) and three trials in which the subject walked, turned and walked back (about 9 steps with each foot).

\subsubsection{Synchronization}

The different measurement systems were synchronized in time by calculating cross correlations. The inertial sensors were synchronized with the reference system by estimating the angular velocity of the shoes based on the three marker positions. The range was estimated with the reference system as well and used to synchronize with the ultrasound range estimates [86]. The times 


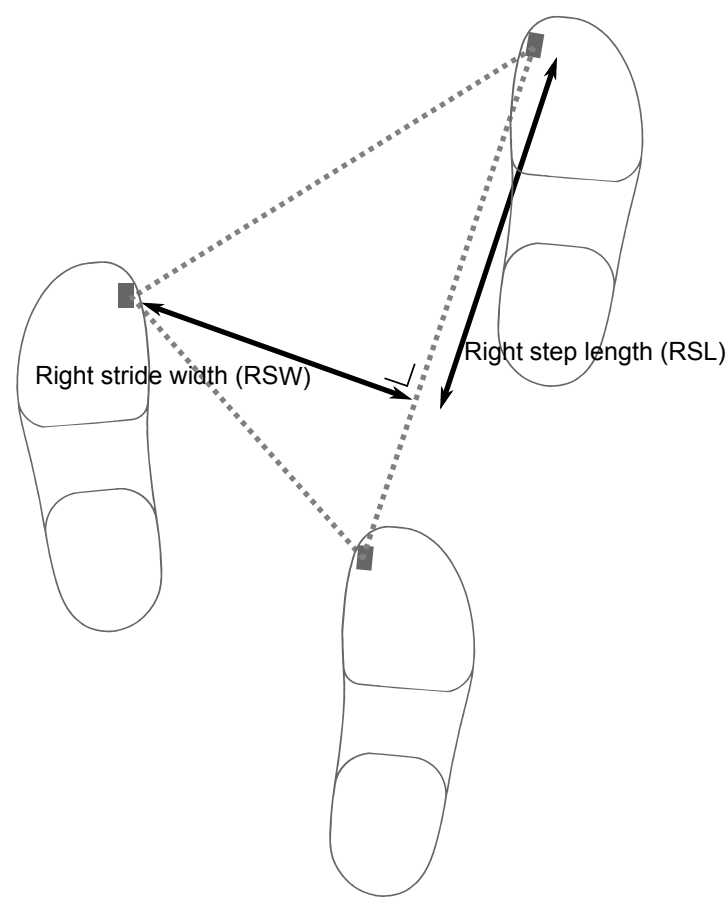

Figure 5.5: Step length and stride width are calculated from positions of ultrasound transducers during stance using the cosine rule. See also Huxham et al. [32]. The advantage of this method is that, in addition to walking in a straight line, it can be used for other movements as well.

for which both cross correlations were maximal were used to synchronize all systems.

\subsubsection{Step length and stride width estimation}

For both right and left foot, step lengths (RSL and LSL respectively) and stride widths (RSW and LSW respectively) were calculated using methods in Huxham et al. 32, see Fig. 5.5. The points on the shoes that were used were the ultrasound transducers. These positions were calculated for the reference system [86] as well as for the filter positions (5.31) and the absolute difference was calculated for a comparison between the two. To obtain a quick insight in the distribution of the absolute differences, boxplots were produced. In these boxplots, the central mark of the boxes is the median, the edges of the boxes are the 25 th and 75 th percentiles ( $q 1$ and $q 3$ ). Whiskers extend to the most extreme data points that are not considered outliers. The maximum whisker length that was used in the plots is $1.5 \cdot I Q R$, with $I Q R$ the interquartile range $(q 3-q 1)$. Outliers were not removed from the comparison. 


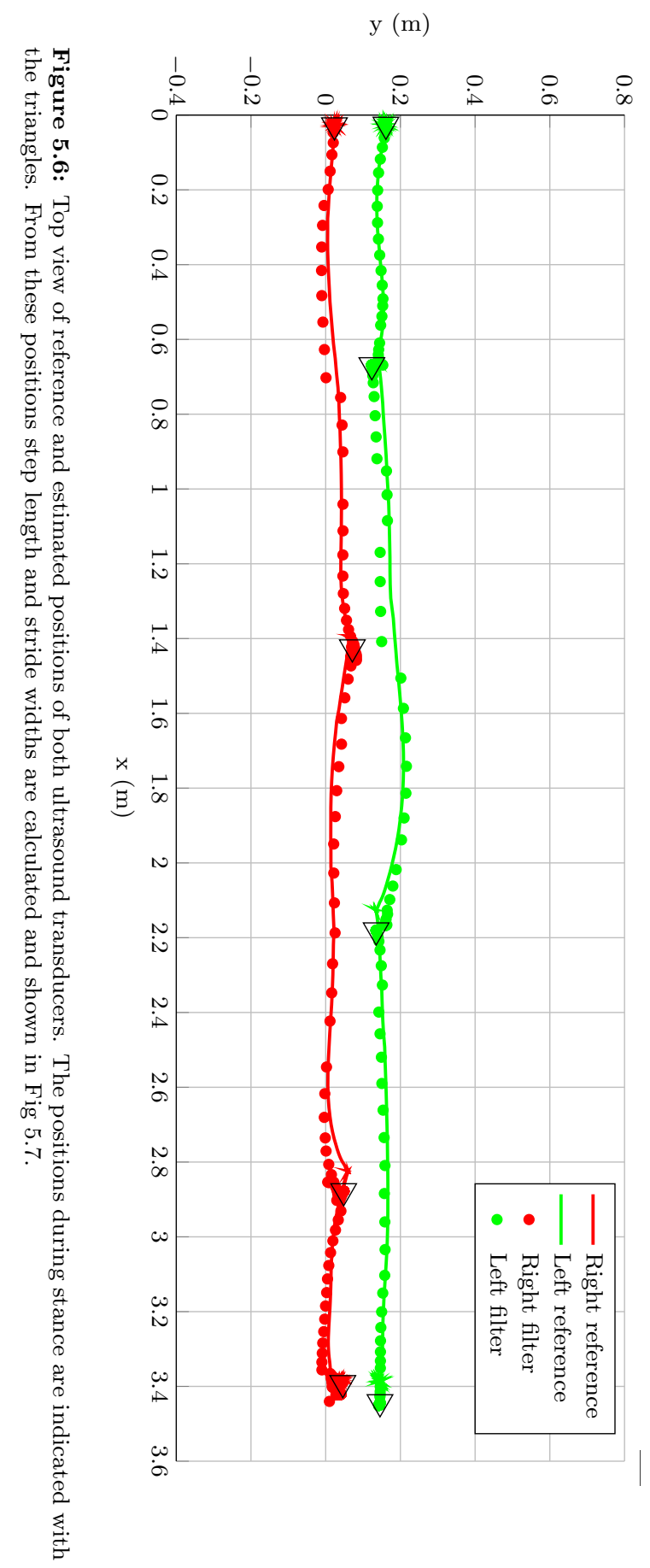


Table 5.1: Process noise and measurement noise standard deviations (std). The uncertainty of the ultrasound range updates $\left(e_{\text {range }}\right)$ is increased for low signal to noise ratios.

\begin{tabular}{ccccccc}
\hline & $\boldsymbol{e}_{G}$ & $\boldsymbol{e}_{A}$ & $\boldsymbol{w}_{b}$ & $\boldsymbol{e}_{v 0}$ & $e_{h}$ & $e_{\text {range }}$ \\
$\mathrm{rad} / \mathrm{s}$ & $\mathrm{m} / \mathrm{s}^{2}$ & $\mathrm{rad} / \mathrm{s}$ & $\mathrm{m} / \mathrm{s}$ & $\mathrm{m}$ & $\mathrm{m}$ \\
\hline $\mathrm{std}$ & $1 \cdot 10^{-2}$ & $1 \cdot 10^{-3}$ & $1 \cdot 10^{-4}$ & $7 \cdot 10^{-2}$ & $5 \cdot 10^{-2}$ & $9 \cdot 10^{-3}$ \\
\hline
\end{tabular}

Table 5.2: Mean and standard deviations (std) of absolute difference between estimated and reference step lengths (SL) and stride widths (SW) calculated from positions during stance. Results are from 54 trials of walking in a straight line with a total of 284 walking steps. See also the corresponding box plots in Fig. 5.8

\begin{tabular}{llllll}
\hline & \multicolumn{4}{c}{ absolute difference $(\mathrm{m})$} \\
\cline { 2 - 3 } Parameter & \multicolumn{2}{c}{ with range update } & & without range update \\
\cline { 2 - 3 } \cline { 5 - 6 } mean & std & & mean & std \\
\hline SL & 0.017 & 0.018 & & 0.056 & 0.047 \\
SW & 0.012 & 0.012 & & 0.021 & 0.019 \\
\hline
\end{tabular}

\subsubsection{Process noise and measurement noise parameters}

The process noise and measurement noise standard deviations (std) are derived from sensor specifications and static measurements and are shown in Table 5.1 . The uncertainty of the ultrasound range updates $\left(e_{\text {range }}\right)$ is increased for low signal to noise ratios as described in the previous chapter [86].

\subsection{Validation results}

In Fig. 5.6 a top-down view of the reference and estimated positions is shown for one walking trial. The positions during stance are indicated with the triangles. There are some differences in global positions, but the differences in relative positions during double stance, from which the step lengths and stride widths are calculated are small (Fig. 5.7).

Fig. 5.8 shows a box plot of the absolute difference between estimated and reference step lengths and stride widths for all 54 walking trials (284 steps). Table 5.2 shows the mean and standard deviations, the step lengths and stride widths that were plotted as outliers in the boxplots are not removed from the comparison. The results are also shown when the ultrasound range update is not applied. It should be noted, however, that the initial foot positions are estimated based on the ultrasound range estimation, as described in Section 5.2.3. No significant differences between left and right were observed.

The results of the trials in which the subject walked around in the measurement volume of the Vicon ${ }^{\circledR}$ system and walked with a turn are combined and shown in Fig. 5.9 and Table 5.3 . the step lengths and stride widths that were plotted as outliers in the boxplots are not removed from the comparison. No 

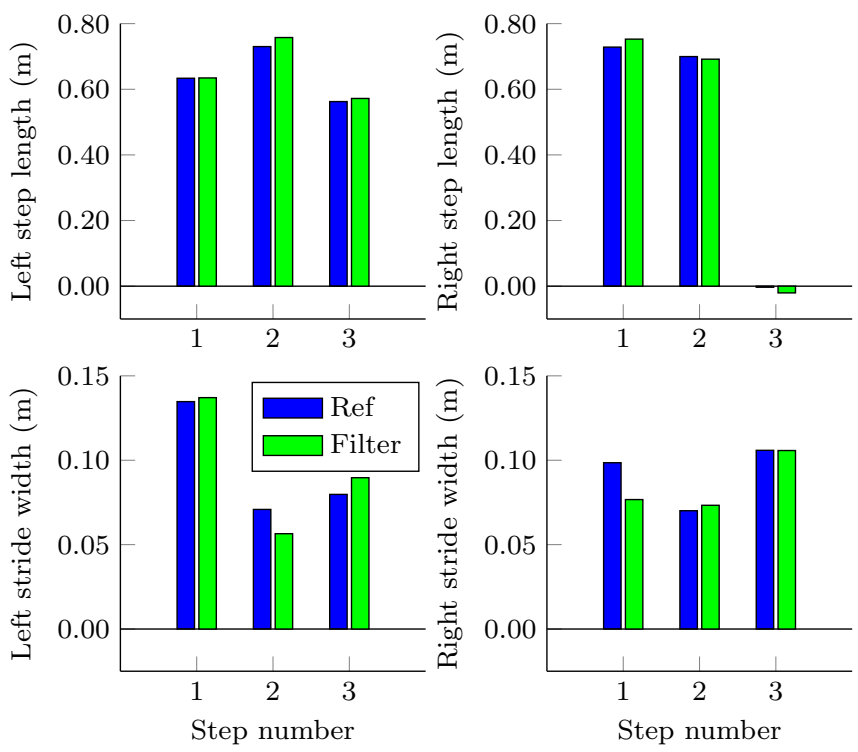

Figure 5.7: Step lengths and stride widths for the walking trial in Fig. 5.6 The third right step length is approximately zero, because this is the last step which ends next to the left foot.

significant differences in results were observed between these two types of walking. The absolute differences are slightly larger than when walking straight. The differences when no ultrasound range update is applied are twice as large as when walking in a straight line. This can be explained because these trials contain more walking steps and differences in step lengths and stride widths increased with time (the feet drift apart). The longer the subject is walking, the larger the differences become, up to differences over $31 \mathrm{~cm}$. No outstanding differences were observed between the results of left and right step lengths and stride widths.

Table 5.3: Mean and standard deviations (std) of absolute difference between estimated and reference step lengths (SL) and stride widths (SW) calculated from positions during stance. Results are from two trials in which the subject walked around in the Vicon ${ }^{\circledR}$ measurement volume (about 14 steps with each foot) and three walk-turn-walk trials (about 9 steps with each foot) with a total of 116 walking steps. See also the corresponding box plots in Fig. 5.9

\begin{tabular}{llllll}
\hline & \multicolumn{3}{c}{ absolute difference $(\mathrm{m})$} \\
\cline { 2 - 3 } Parameter & mean & std & & mean & std \\
\cline { 2 - 3 } \cline { 5 - 6 } SL & mith range update & & without range update \\
\hline SW & 0.017 & 0.020 & & 0.109 & 0.071 \\
& 0.015 & 0.015 & & 0.090 & 0.062 \\
\hline
\end{tabular}




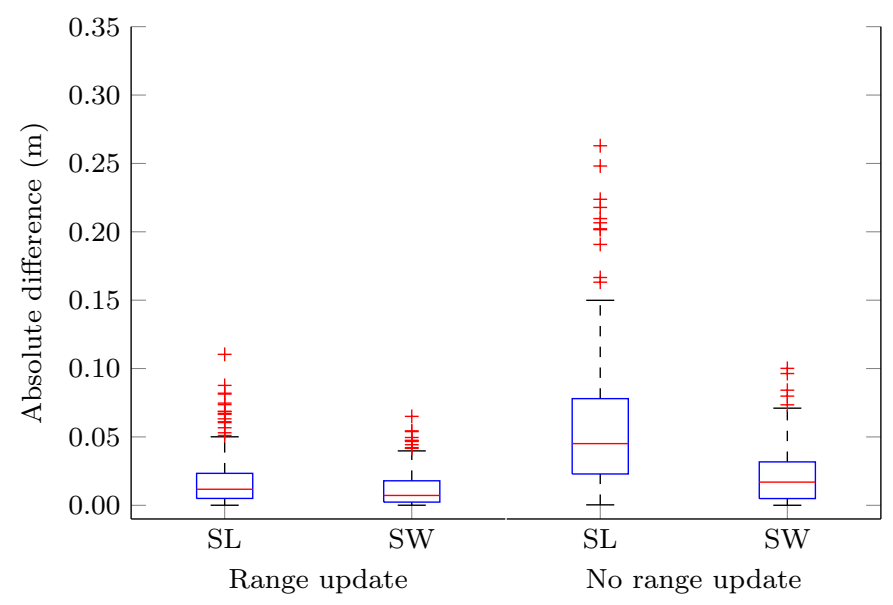

Figure 5.8: Results from 54 trials of walking in a straight line with a total of 284 walking steps. Results are presented in box plots of absolute difference between estimated and reference step lengths (SL) and stride widths (SW) calculated from positions during stance. The central mark is the median, the edges of the boxes are the 25 th and 75 th percentiles. Whiskers extend to the most extreme data points that are not considered outliers (outliers are plotted as + ). See also Table 5.2

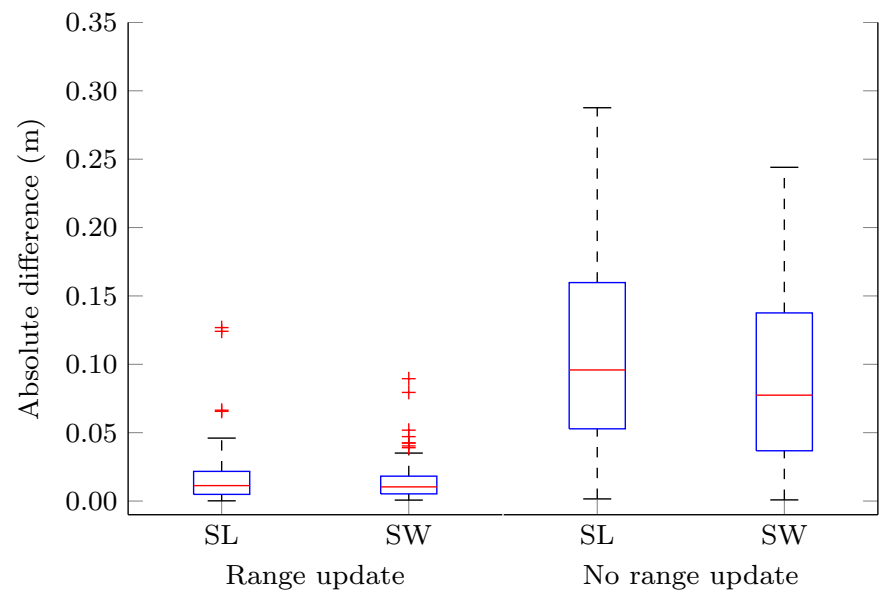

Figure 5.9: Results from two trials in which a subject walked around in the Vicon ${ }^{\circledR}$ measurement volume and three walk-turn-walk trials with a total of 116 walking steps. Results are presented in box plots of absolute difference between estimated and reference step lengths (SL) and stride widths (SW) calculated from positions during stance. The central mark is the median, the edges of the boxes are the 25th and 75 th percentiles. Whiskers extend to the most extreme data points that are not considered outliers (outliers are plotted as + ). See also Table 5.3 


\subsection{Discussion}

It is shown that relative foot positions can be estimated accurately using only two inertial sensors and two ultrasound transducers. The system is small, light weighted and can be implemented on every shoe. In addition to estimating relative foot positions, $3 \mathrm{D}$ velocities and orientations are also estimated and stance and swing times of each foot are available due to the contact detection, making it a complete ambulatory gait analysis system.

The presented results are a comparison of the filter with and without range update. It should be noted, however, that without the ultrasound range estimation, the initial distance between the feet is unknown. Therefore it is not possible to estimate relative foot positions without these ultrasound range estimates (or other range or position estimates).

Step lengths and stride widths are calculated from the estimated positions of the ultrasound transducers. In literature they are often calculated from the heel positions. This is also possible for our measurements, since the orientations of the feet and the length of the shoes are known. The ultrasound transducers were mounted in the forefeet of the Xsens ForceShoes $^{\mathrm{TM}}$, because there was enough space and there was a bolt to mount them to easily. For the line of sight during walking the position of the transducers on the shoes does not matter, as long as they are pointed inwards.

With the walking around and walking with a turn trials we have shown that the presented system can be used for activities other than walking in a straight line. In theory it should also be possible to walk on for example stairs, however the height update should be turned off in this case. This is nonetheless not investigated in this study. It should also be noted that line of sight between the ultrasound transducers can become an issue, although the system can continue for several walking steps without range updates in this case. Afterwards the positions can be updated backwards in time for the time steps in which no range estimates were available.

The height update can be switched off in case a subject is not walking on a flat floor. In this case the feet can drift slowly up or down, however they do not drift apart due to the range update. When the height update is not used for the trials in which the subjects walk in a straight line, the mean absolute differences for step lengths and stride widths are slightly larger, namely $1.9 \mathrm{~cm}$ (std $1.8 \mathrm{~cm}$ ) and $1.3 \mathrm{~cm}$ (std $1.4 \mathrm{~cm}$ ) respectively. Alternatively a height update using barometric pressure sensors could be used, since these sensors are included in for example the Xsens MTw motion trackers 93 .

Currently we used the inertial sensors in the Xsens ForceShoes ${ }^{\mathrm{TM}}$, which allow a maximum sample frequency of $50 \mathrm{~Hz}$. We think even better results can be obtained when sampling at higher frequency, especially due to fast changes in accelerations during impact.

An addition to the presented method could be the use of the inertial sensors in the heel segments of the ForceShoes ${ }^{\text {TM }}$. In combination with the force/moment sensors, this could than be used to estimate center of mass with 
respect to the relative positions of the feet and hence be a powerful tool for assessment of balance during daily life activities.

The speed of sound, and hence the ultrasound range estimates are temperature dependent. Currently it is assumed that the temperature remains constant during a set of measurements. When walking out of a heated room, for example, this could immediately lead to an error in the estimated distance. A rule of thumb is that for a $10{ }^{\circ} \mathrm{C}$ change in temperature, the speed of sound and hence the estimated distance, changes with approximately $2 \%$ (see (5.4) and [57]). To overcome this problem a temperature sensor could be added to the system.

The initial heading is currently estimated off-line by running the filter once without the ultrasound range updates. The change in position of each inertial sensor is then used for estimating the initial heading. This heading was compared to the heading estimated from the optical markers and showed good correspondence. The range update also provides heading information, because the relation between position and orientation error in matrix $\boldsymbol{F}(5.19)$. This was tested by applying an incorrect heading to several trials, after a few steps the (relative) heading was corrected automatically. However, since only a few steps are available in our measurements, we chose to estimate the heading offline. The time synchronization of the different systems is currently also done off-line by estimating cross correlations. If signals from sensors are received in real-time, it is also possible to run the filter in real-time.

For the initial positions, the same holds as for the initial heading. For the current measurements, the subjects started with their feet next to each other (the initial $x$-position for both feet was set to zero). This was also varied for some trials by changing the initial $x$-positions and after a few steps the relative positions were automatically corrected due to the range update. It should be noted that the ultrasound outlier detection in 5.35 should be switched off during the first steps if the initial position and heading of the feet is unknown.

The fusion of the inertial and ultrasound sensors was done using an extended Kalman filter with orientation error and gyroscope bias error states. This way, the equations can be described as linear processes and the computation load is smaller when compared to for example a particle filter. We compared the estimated orientation with the orientation estimated from the optical reference system. No large differences were observed between both orientations. For results that are easier to interpret and for an easier calculation of step lengths and stride widths, we chose to include the absolute position of both feet in the state vector $\boldsymbol{x}$. Alternatively the relative position between the feet could be included and three states less are required.

It should be noted that the presented system is not useful for estimating global positions, for example for navigation. Accumulative errors (drift, due to integrating accelerations) in each foot are translated through the range update to the other foot (as can be seen in Fig. 5.6). If wanted, these accumulative errors can be reduced using stationary ultrasound beacons on the floor, as for example in 30. Other position aiding systems can be fused with the inertial sensors as well [62, for example ultra wide band [29] or GPS [18]. 


\subsection{Conclusion}

By fusing ultrasound range estimates with inertial sensors, relative positions of feet can be estimated accurately and ambulatorily. The presented extended Kalman filter was validated in three subjects using an optical reference. Mean absolute difference for step lengths was $1.7 \mathrm{~cm}($ std $1.8 \mathrm{~cm})$ and for stride widths $1.2 \mathrm{~cm}$ (std $1.2 \mathrm{~cm})$, when walking in a straight line for about 6 steps. When no range update is applied, the mean absolute differences for step lengths and stride widths are significantly larger due to integration drift, that is, 5.6 $( \pm 4.7) \mathrm{cm}$ and $2.1( \pm 1.9) \mathrm{cm}$ respectively. Furthermore, it should be noted that relative positions cannot be estimated at all when beginning positions of inertial sensors are unknown.

In addition to walking in a straight line, the presented filter was tested for walking around the measurement volume of the optical reference system and walking with a turn. With mean absolute differences for step lengths $1.7 \mathrm{~cm}$ (std $2.0 \mathrm{~cm}$ ) and $1.5 \mathrm{~cm}$ (std $1.5 \mathrm{~cm}$ ) for stride widths, it can be concluded that the system is suitable for accurately estimating relative foot positions during variable gait outside a laboratory.

In addition to estimating relative foot positions, velocities, orientations and stance and swing times are also estimated, providing a complete description of footstep kinematics and timing. 


\section{Chapter 6}

\section{Ambulatory assessment of walk- ing balance after stroke}

प

In preparation:

F. B. van Meulen, D. Weenk, J. H. Buurke, B. J. F. van Beijnum, P. H. Veltink Ambulatory assessment of walking balance after stroke 


\section{Abstract}

For optimal guidance of the rehabilitation therapy of stroke patients in an in-home setting, a small and easy to use wearable system is needed. In this chapter we present a shoe-integrated system that qualifies walking performance during activities of daily living. Data from 3D force and torque sensors, 3D inertial sensors and ultrasound transducers are fused to estimate 3D (relative) position, velocity, orientation and ground reaction forces of each foot. From these estimates, center of mass and base of support are derived together with a dynamic stability margin, which is the (velocity) extrapolated center of mass with respect to the front-line of the base of support in walking direction. Two 10 meter walk tests were recorded from 13 participants who suffered a stroke. The first and last two steps of each trial were removed after which the mean of the dynamic stability margin was calculated for each participant. Especially participants with a lower Berg Balance Scale score show a negative (center of mass is within the base of support) or small-positive dynamic stability margin and a low walking speed. Less impaired patients showed higher walking velocities and higher stability margins. With the presented ambulatory assessment methods, daily-life walking performance can be qualified. 


\subsection{Introduction}

T MPAIRED WALKING BALANCE commonly follows a stroke, which reduces the patient's ability to walk and hence their independence in daily-life [54]. Clinical assessment methods of walking balance have been developed to grade a patient's ability to walk (independently) after stroke 55. For a better guidance during the rehabilitation of walking, medical professionals need a system to qualitatively evaluate balance and to estimate quantitative parameters to describe the movements of the patients' feet and body center of mass (CoM) [23, 84. Such a measurement system preferably has small-embedded sensors which should have no influence on normal daily-life behavior [5].

During forward walking, the CoM is moving within the area between both feet (i.e. base of support, BoS). To evaluate a persons' stability during walking the extrapolated center of mass (XCoM) can be calculated, which is the position of the CoM extrapolated using the velocity of the CoM. A person will be dynamically stable when the vertical projection of the XCoM on the ground, is within the BoS [26, 43, 44]. Moments of dynamic instability regularly occur during walking. These moments are followed by another step to prevent a fall [26, 44]. Objective evaluation of stability parameters during (training of) walking, may result in fewer incidences of falling.

To continuously assess the dynamic stability of a person, information on the position of the $\mathrm{XCoM}$ relative to the BoS is necessary. For a continuous evaluation of the BoS, movements of both feet relative to each other are required. A feasible method for movement assessment in a daily-life setting is the use of inertial measurement units (IMUs). This allows easy assessment of foot movements in a daily-life setting, without the use of an external physical reference system [77. Previous studies reported on the use of IMUs for the estimation of qualitative and quantitative parameters of walking and balance performance, such as cadence, stride length and velocity [56, 64, 84. However, using only IMUs it is not possible to evaluate parameters which depend on the relative position of both feet, such as step length, step width and size of the BoS, due to the drift in position estimation. Furthermore, only position changes from each IMU can be calculated and since the begin positions are unknown, this does not result in relative positions of the feet. We solved this problem by fusing IMUs and an ultrasound system to directly estimate the relative distance between both feet 84 .

For a continuous evaluation of CoM positions as well as the XCoM, positions of both feet, and ground reaction forces (GRF) beneath both feet, should be known. For the estimation of the GRF beneath both feet, traditionally, multiple force plates or sensorised walkways are used in a lab situation [44, 52. These systems mostly cause restriction in movements or are only able to measure one or two steps. For the evaluation of forces underneath both feet during daily-life activities, more and more we see the introduction of shoes instrumented with force or pressure sensors [11, 42, 63, 67, 75. These systems allow ambulatory estimation of ground reaction forces, making it suitable for moni- 
toring multiple steps and walking with changes in walking direction. However, as far as the authors know, there is no system available which allows assessing dynamic stability in a daily-life setting. Such a system would require an ambulatory estimation of foot orientations, relative foot positions and ground reaction forces simultaneously.

The objective of this study is to qualify walking patterns of stroke patients in an ambulatory setting, by combining instrumented shoes (integrated force and inertial sensing) and ultrasound transducers. The dynamic stability margin, as well as other (temporal, kinematic and kinetic) gait parameters will be estimated using the combination of these different wearable measurement systems. Using these parameters, walking performance and stability of stroke subjects can be assessed and monitored in a daily-life setting.

\subsection{Method}

\subsubsection{Participants}

For this study seventeen stroke patients from Roessingh rehabilitation hospital, located in Enschede, the Netherlands were recruited. Recruited participants were between 35 and 75 years of age and had a hemiparesis as a result of a single unilateral stroke, diagnosed at least six months earlier. Exclusion criteria were inability to perform given instructions, inability to understand questionnaires and a medical history with more than one stroke. The study protocol is a subset of a larger protocol approved by the local medical ethics committee [7]. All participants signed written informed consent before participating.

Two participants with severely affected lower extremity function were not able to complete the task without assistance due to unstable walking patterns. The corresponding test results were excluded from the analysis. Data of two other participants were not fully recorded because of a broken cable during the session or sensors which were not properly functioning. Remaining were 13 participants ( 8 male) with an average age of $63.8(\mathrm{SD} \pm 8.9)$ years, $2.3(\mathrm{SD}$ \pm 1.8 ) years post stroke. Participants' balance was assessed using the Berg balance scale (BBS) 4]. Participant specific information is reported in Table 6.1 and includes gender, age, number of years post stroke, dominant and affected side, weight, height, BBS score and whether or not a walking aid is used during activities of daily living.

\subsubsection{Experimental protocol}

Participants walked twice at a self-selected comfortable pace along a 10 meter path, without the use of any walking assistance device. Walking speed was estimated using a stopwatch. GRF of the heel and forefoot segments of both feet were evaluated using the Xsens ForceShoes ${ }^{\text {TM }}$ [67]. Each of the four segments 


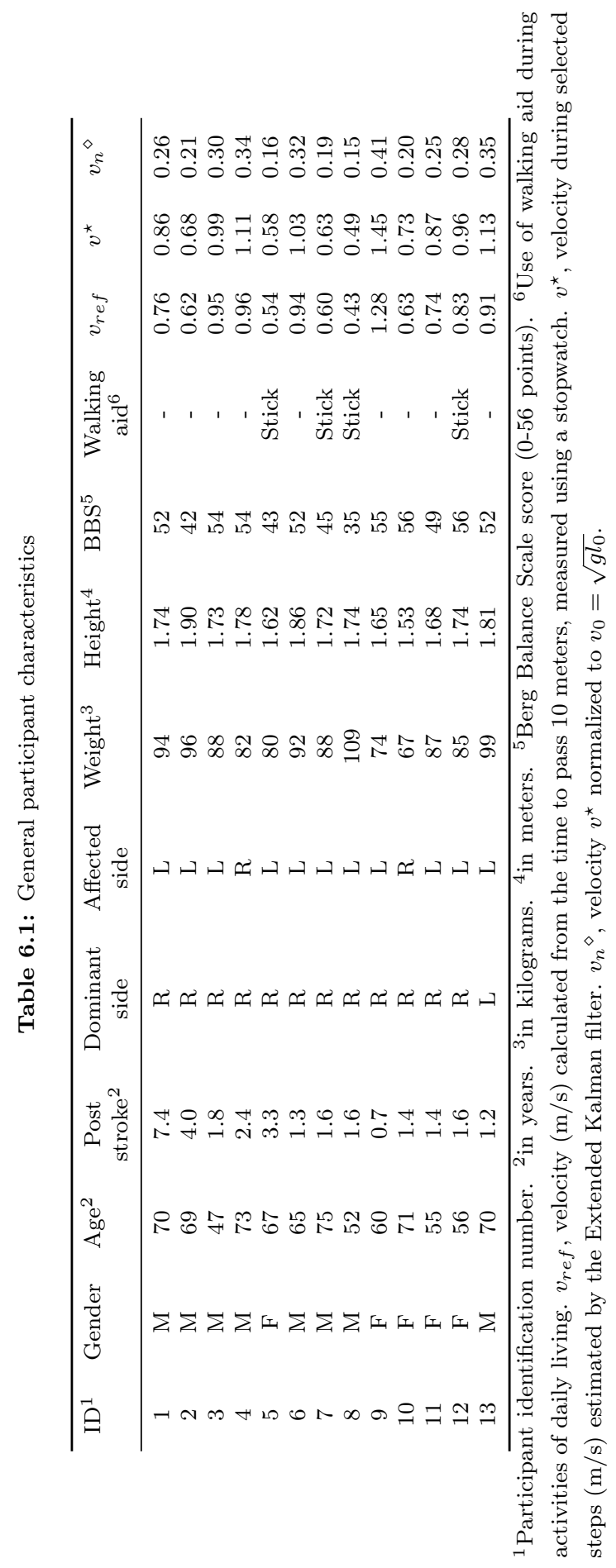




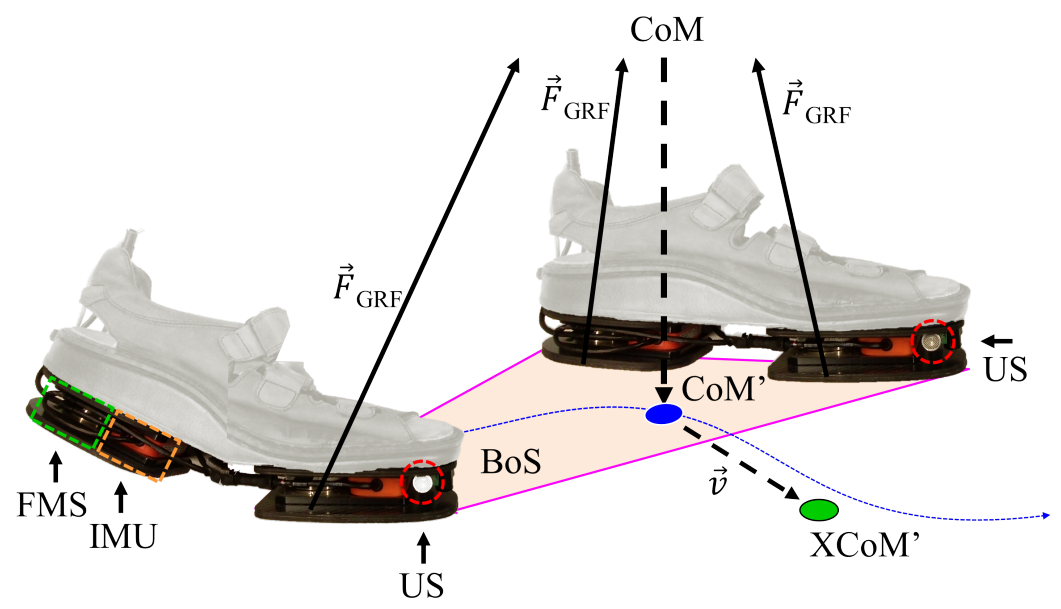

Figure 6.1: In this study Xsens ForceShoes ${ }^{\mathrm{TM}}$ were used, containing one inertial measurement unit (IMU, dashed orange square) and one force/moment (FMS, dashed green square) sensor per heel or forefoot segment. Near the inertial sensor in the forefeet an ultrasound transducer (US, dashed red circle) was mounted in each shoe. Kinematic and kinetic data were used to estimate the position of the center of mass (CoM - blue circle) relative to the position of both feet, the projection of the center of mass on the ground (CoM') within the base of support (BoS) and the extrapolated CoM (XCoM' - green circle)

contain one combined inertial and magnetic sensor and one 3D force/moment sensor, see Figure 6.1. Data of these eight sensors was collected with a sample frequency of $50 \mathrm{~Hz}$. Data of the magnetometers were not used. In addition, the distance between the feet was estimated using two ultrasound transducers that were mounted near the inertial sensors in the forefoot segment (Figure 6.1). By measuring the time of flight of a $40 \mathrm{kHz}$ pulse, that was sent from one shoe to the other, the distance between both shoes was estimated. The distance was estimated approximately 13 times each second [86].

\subsubsection{Data processing}

All data were processed offline and analyzed using MATLAB ${ }^{\circledR}$ (MathWorks Inc., Natick, MA). Relative foot positions, orientations and velocities were estimated using an extended Kalman filter. The filter fuses 3D accelerations, $3 \mathrm{D}$ angular velocities and range estimates from only the forefeet segments. A detailed description of the used fusion algorithms is presented in [84]. In short, the $3 \mathrm{D}$ position, velocity and orientations of right and left forefoot are predicted using the inertial sensor data. After this prediction, height-, zero velocity- and ultrasound range- updates are performed.

The participants' BoS was defined by the area between all foot segments 
which were in contact with the ground and the participant was supporting on (estimated by thresholding the magnitude of the 3D force at 20 Newton).

The position of the center of pressure $(\mathrm{CoP})$ per foot was estimated using measured forces and moments of both force/moment sensors of one foot, as described in 69. By combining relative foot positions with $\mathrm{CoP}$ and GRF of each foot, the total CoP was estimated. Position of the CoM was obtained using methods of Schepers and others [69, in which the CoM position estimation is a summation of the low-pass filtered component of the total CoP movement and the high pass filtered component of the double integrated CoM acceleration. Finally, during contact of both feet (support on at least one segment of both feet), the XCoM' was calculated as [26]:

$$
\mathrm{XCoM}^{\prime}=\mathrm{CoM}^{\prime}+\frac{\mathrm{CoM} v}{\omega_{0}}
$$

with CoM' the position of the vertical projection of the CoM on the ground, $\mathrm{CoM} v$ the velocity of the CoM in the transversal plane and $\omega_{0}=\sqrt{g / l_{0}}$, in which $g=9.81 \mathrm{~m} / \mathrm{s}^{2}$ (earth gravitational acceleration) and $l_{0}$ the greater trochanter height, as we estimated from a proportion of the total body height [14.

\subsubsection{Data analysis}

Using the complete kinematic and kinetic reconstruction during walking, temporal, kinematic and kinetic parameters can be calculated. Data of only quasicyclical walking is used for the calculation of the parameters. Therefore, from each walking trial of 10 meters, the first two and last two steps were removed. For both walking trials of each participant the mean of all parameters was calculated. To be able to compare different participants, parameters were normalized to body size as described in [27].

\section{Stability margins}

The static and dynamic stability margins (SSM and DSM, respectively) were calculated as the shortest distance from the CoM and XCoM to the front line (in the walking direction) of the BoS [43, 26], at each instant in the double support phase of the gait cycle. Figure 6.2 shows a top down view of four consecutive steps of a walking trial of participant number 5. In this figure (X)CoM and the front-line of the BoS just before heel-off, including the shortest distance between them (SSM/DSM), are indicated. We define negative values of SSM and DSM as static and dynamic stable respectively, meaning CoM and XCoM respectively are in inside the BoS. Therefore, positive values of these stability margins indicate instability. 


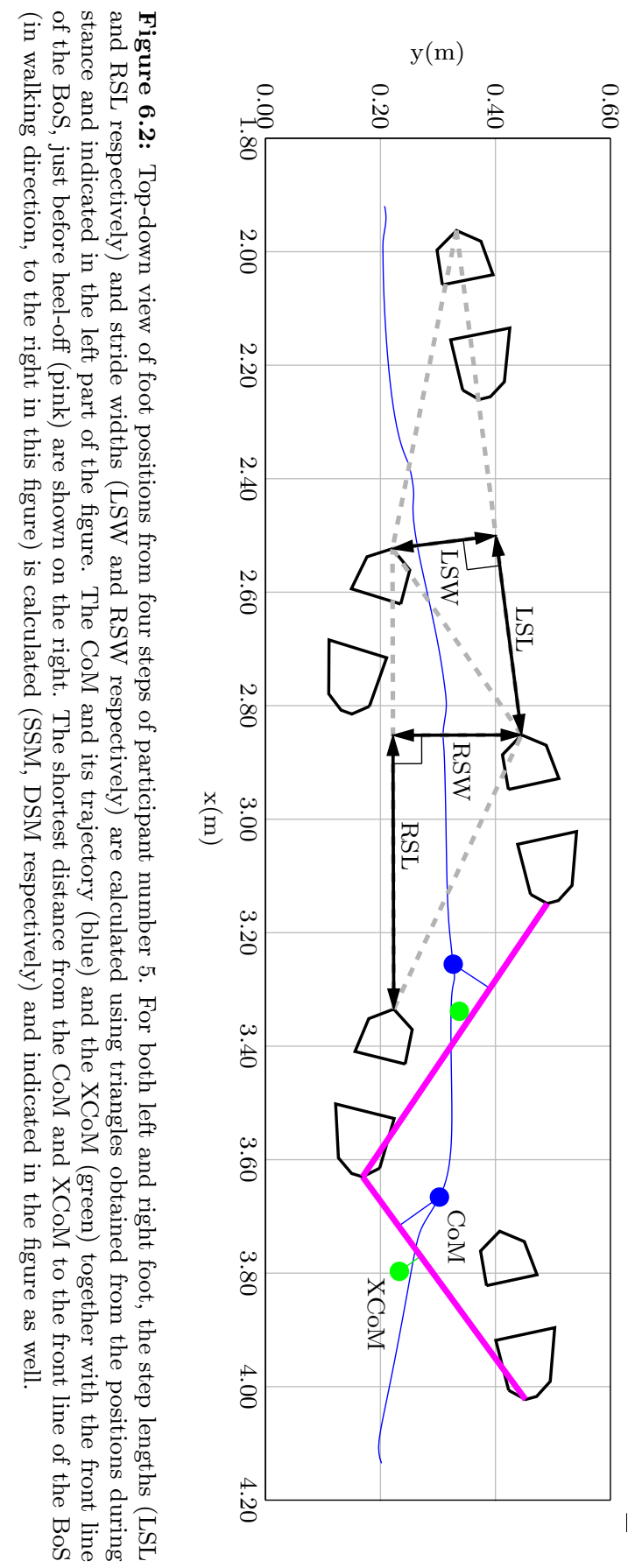




\section{Other (temporal and kinematic) gait parameters}

Based on thresholding the forces as described above, stance and swing times were calculated. From the estimated 3D-positions of left and right foot, step lengths (LSL and RSL respectively) and stride widths (LSW and RSW respectively) were calculated using the method as described by Huxham and others 32. In Figure 6.2 the definitions of step lengths and stride widths are given. The results of these gait parameters are not reported in this chapter, were the focus is on the stability margins.

\subsection{Results}

Table 6.1 shows for each participant the mean velocity during the selected steps (after eliminating the first and last two steps) as estimated by the Extended Kalman filter. This velocity is normalized to $v_{0}=\sqrt{g l_{0}}$, as described in 27]. As a reference, the velocity estimated from the stopwatch of the complete 10 meter walk test is also listed.

Figure 6.3 shows the stability margins of participant \#5, during the selected

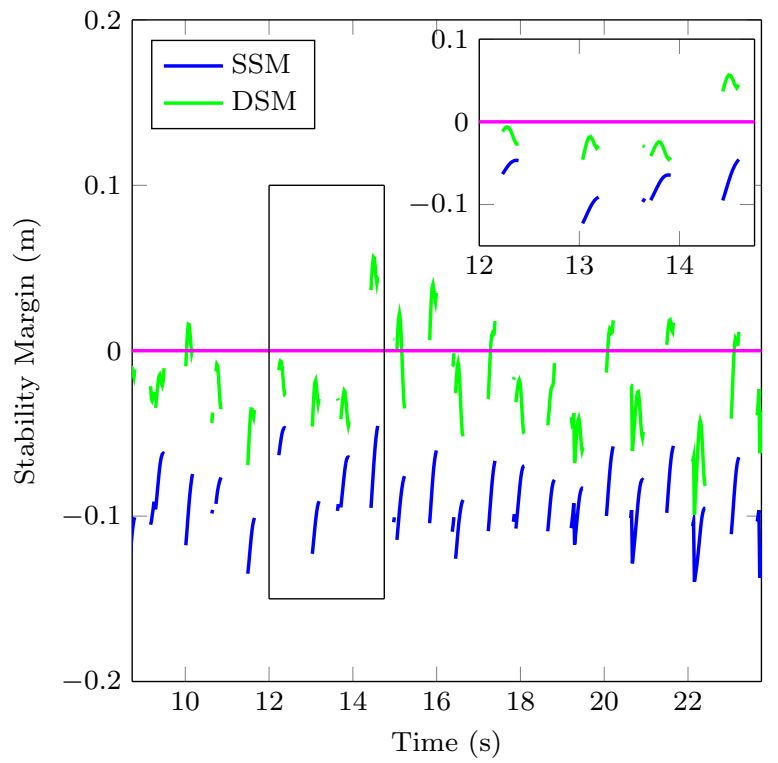

Figure 6.3: The SSM (blue) and the DSM (green) evaluated during the double stance phases for one walking trial of participant number 5 . Negative values indicate that the $(\mathrm{X}) \mathrm{CoM}$ is inside the BoS. Mean SSM for this trial was $-0.11 \mathrm{~m}$, mean DSM was $-0.02 \mathrm{~m}$. The inset is a magnification (indicated by the box) and corresponds to the time window (12-14.75 s) of which the steps are shown in Figure 6.2 


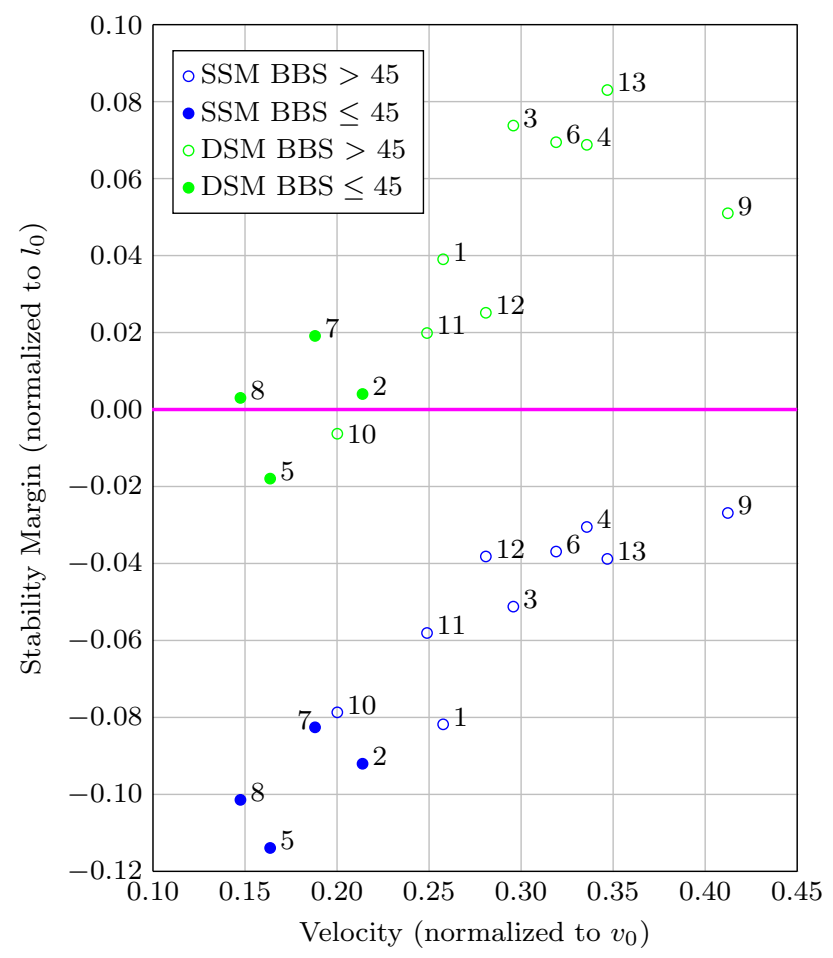

Figure 6.4: Mean SSM (blue) and DSM (green) (normalized to $l_{0}$ ) versus velocity (normalized to $\left.v_{0}\right)$ estimated by the filter $\left(v_{n}\right.$ in Table 6.1), for all 13 participants (indicated with the numbers). Filled data markers are of those participants with a BBS score below or equal to 45 .

steps of a single walking trial, over time. If one foot is in swing phase, i.e. the $\mathrm{BoS}$ is restricted to the size of only the other foot, no estimation of SSM and DSM is made which is represented as a gap in both signals of Figure 6.3. The four steps that are shown in Figure 6.2 are indicated by the rectangular box and a zoom of these steps is shown in the inset of the figure.

The normalized mean SSM and DSM were estimated for both walking trials of each participant and related to participant's average walking speed, as shown in Figure 6.4 The mean SSM is negative, i.e. within the BoS, for all participants. Of most participants, the average DSM is positive, i.e. outside the BoS. Especially participants with lower BBS scores (filled bullets in Figure 6.4 show a lower walking speed, a more negative SSM and small positive as well as negative DSM. Less impaired patients $(\mathrm{BBS}>45)$ showed higher walking velocities and higher DSM values. 


\subsection{Discussion}

The objective of this study was to qualify walking patterns of stroke patients in an ambulatory setting. By combining Xsens ForceShoes ${ }^{\text {TM }}$ and ultrasound modules, we were able to completely reconstruct kinetics and kinematics of both feet as well as the position of the CoM relative to both feet, without the use of a lab restricted setup. By extrapolating the CoM using the transversal velocity of the CoM, the XCoM was estimated. This XCoM can be used to examine the walking patterns of stroke subjects, by evaluating the distance between XCoM and the front-line of the BoS (i.e. DSM). Overall, less-affected participants with higher BBS scores show a higher walking speed and larger DSM values. While more-affected participants - especially the ones with BBS score of 45 or lower, who have a higher risk of falling - show smaller velocities and smaller and even negative values for their DSM. In case of a negative mean DSM value during walking, a person is dynamic stable during walking, which means that after each step made, no extra step is needed to prevent a fall (on average). Knowing the size of patients' DSM during walking, it might be possible to objectively follow up progression or deterioration of walking performance. Furthermore, this information may be of importance during the training of controlling the position of the $\mathrm{XCoM}$ with respect to the BoS, which might be one prerequisite for preventing falls during walking [24].

An important limitation of our study are the sizes of the used sensors, which were integrated in the instrumented shoes. Although previous research found only limited influence on walking patterns of patients with knee osteoarthritis while wearing these instrumented shoes [75, the shoes are relatively heavy $( \pm 1 \mathrm{~kg}$ per shoe) and the sole height is relatively high $( \pm 2.5 \mathrm{~cm})$. Current technical developments may result in the use of smaller force/moment sensors [9] integrated in shoes and the already widely available smaller inertial sensors, for a more general acceptance of the system [5]. Nevertheless, the number of sensors may be reduced depending on the actual research question. We presented a system using eight sensors (data of the inertial sensors in the heel part of the shoes is not used), which are all required for the dynamic balance parameters shown in Figure 6.3 and Figure 6.4 Using these sensors, or a reduced set of sensors, many other objective parameters can be examined. For example, for the estimation of stance and swing times, a system with only inertial sensors or force sensors could be used. For the evaluation of step lengths and step widths a system with inertial sensors in combination with ultrasound transducers suffices, as was shown in [87]. Inertial sensor data of the heel segments can be used to additionally examine orientations of heel segments, the rolling of the feet or evaluation of heel contact.

We demonstrated a method to qualify walking patterns in stroke subjects during straight line walking at a continues speed, in an ambulatory setting. Using the described setup it is possible to evaluate other phases of walking, such as starting, stopping, standing, transfers, turning around and non-repetitive walking patterns. Especially in an ambulatory setting, the stability of a stroke 
patient during these phases might be of interest, because of the high incidence of falls during these non-stationary walking phases [12]. 
Chapter 7

Conclusions and discussion 


\subsection{Conclusions}

We are now five 'steps' closer to a click-on-and-play human motion capture system, one step is made in each of the chapters 2 till 6 . Chapter 2 and 3 contributed to the achievement of the first project goal, the automatic sensor to segment identification. The second project goal, relative foot position and orientation estimation and the assessment of balance, is accomplished in the chapters 4 till 6 . This section describes how these goals have been accomplished.

\subsubsection{Automatic sensor to segment identification}

The first project goal was to develop an algorithm for the automatic identification of the body segments to which IMUs are attached.

In chapter 2 an algorithm was presented to identify which IMU is located on which body segment, under the assumption that the subject is walking and that a known IMU configuration is used. This algorithm is capable of distinguishing left and right segments. Cross correlation features between signals of different IMUs were used and the features were ranked. A decision tree was used to identify the segment to which each sensor was attached. When using a full-body configuration (17 different IMU locations), $97.5 \%$ of the sensors were correctly classified. In chapter 3 an algorithm was presented that uses features extracted from data of a single IMU and logistic regression classification. To improve the accuracy of the location identification, the algorithm was divided into three steps. In a first step, periods in which the user is walking are identified. Next, these periods are used for the IMU location identification. Finally, for each IMU location a model was developed for activity recognition. Activities that were used are walking, sitting down, standing up, laying down, writing or typing and jumping. Using this method, there is no need for prior knowledge about the sensor configuration or the activity the user is performing. For a full-body configuration, in the first step $87.2 \%$ of the walking periods were correctly identified. In the second step $83.3 \%$ of the IMU locations were correctly classified. The maximum accuracy that was achieved for activity recognition was $91.7 \%$, when using an IMU located on a shoulder.

Considering the algorithms presented in the chapters 2 and 3 , it can be concluded that the first project goal was accomplished.

\subsubsection{Relative foot position and orientation estimation and balance assessment}

The second project goal was to develop a new sensor systems, with a minimal amount of sensors, for the estimation of relative foot positions and orientations and the assessment of balance during gait. From these estimates, clinically relevant and easy to interpret parameters were derived.

In chapter 4 a system is presented to estimate the distance between the feet using ultrasound time of flight measurements. This system was validated 
using an optical reference system and showed an average error in distance estimation of $7.0 \mathrm{~mm}$. In chapter 5 an easy to use ambulatory gait analysis system with only sensors on the shoes was presented. Instead of IMUs on the pelvis, upper legs, lower legs and feet, this system only requires two IMUs and two ultrasound transducers. In an extended Kalman filter the information from these sensors is fused to estimate 3D (relative) positions, velocities and orientations of both feet. This system was also validated using an optical reference system. The mean absolute differences between both systems were 1.7 and $1.2 \mathrm{~cm}$, for step lengths and step widths, respectively. Regarding the clinically relevant and easy to interpret parameters, in addition to the kinematic parameters, timing parameters and kinetics of gait are investigated in chapter 6. By combining both kinematics and kinetics, balance of stroke patients is assessed during walking. A margin of stability - which is the minimal distance from the extrapolated center of mass (projected on the ground) to the base of support - was defined. Overall the average walking velocity as well as the stability margins were smaller for more-affected participants.

An ambulatory gait analysis system with sensors only on the feet was presented in chapter 4 and 5 . Balance assessment and the calculation of clinically relevant and easy to interpret parameters were described in chapter 6 . Therefore, it can be concluded that the second project goal is achieved as well.

\subsection{Discussion and future perspectives}

\subsubsection{Automatic sensor to segment identification}

As described in chapter 2 and chapter 3 two methods for the automatic identification of inertial sensor locations have been presented. When it is known which activity the user is performing and which sensor-configuration is used, it is recommended to use the method presented in chapter 2. Higher accuracies are achieved with this method when compared to the method from chapter 3. Furthermore, in chapter 2 a method for left and right identification was presented. This method consists of two parts. First, the correlation between the sternum and upper arm IMUs, the pelvis and upper leg IMUs is used to identify left and right upper arms and upper legs respectively. For the remaining IMUs, correlations with IMUs on adjacent segments are used.

When the activity the user is performing and the sensor configuration are unknown, it is recommended to use the method presented in chapter 3 . In this chapter, probabilities are calculated that give measures for the certainty of the location and activity classification outcomes. If a left and right identification is required, the method from chapter 2 should be used. However, cross correlations of signals of different IMUs are needed, making it impossible to run the algorithm on each individual IMU. Future research is needed to investigate the left and right identification based on data from a single IMU.

Compared to other research in literature, the advantages of the automatic 
identification methods presented in this thesis are the possibility to use a fullbody configuration of 17 different on-body locations. The number of recognized IMU sites in other literature is up to 7 [47. Furthermore, whereas most algorithms were trained and tested on healthy subjects, our algorithms were subsequently validated using measurements with ACL and stroke patients [4]. Furthermore, we presented a method to transform the IMU signals to a global coordinate frame - making the algorithm invariant for the orientation of the IMU with respect to the segment - whereas for example Xu et al. 94 extract features from each axis of the IMU, in the IMU coordinate frame. This latter can make the identification sensitive to changes in the orientation of the IMU with respect to the segment to which it is attached.

\subsubsection{Relative foot position and orientation estimation and balance assessment}

In chapters 4 and 5 an easy to use ambulatory gait analysis system, with only sensors on the shoes, is presented. The information from ultrasound range measurements and IMU data is fused to estimate 3D (relative) positions, velocities and orientations of both feet. Currently the time synchronization of the ultrasound range and the IMUs is performed offline using cross correlations. A recommendation would be to combine these sensors in one measurement system to solve this synchronization issue. If one would integrate each ultrasound transducer in an IMU, this issue is solved. Because speed of sound - and hence the estimated distance - is temperature dependent, also a temperature sensor could be included to compensate for this. Furthermore, the distance between the ultrasound transducer and the origin of the accelerometer is known and fixed if both sensors are incorporated in one housing (currently it is a filter parameter). In this way the system becomes really click-on-and-play and the sensors can be easily attached to every shoe or even to other human body segments. Only the position and orientation with respect to the segment needs to be estimated using a sensor-segment calibration.

If joint angles are to be estimated, additional IMUs on the legs and pelvis can be added to the system. With the use of the ultrasound range system, the closed loop information can then be used to improve lower body kinematic estimation. If ultrasound transducers are included in every IMU even more relative positions are available and kinematic estimation can be improved even further.

Instead of using ultrasound for foot position estimation, Hung and Suh 31 presented a system with a camera installed on one foot and infrared LEDs on the other foot. The mean position error of their system was $1.2-2.5 \mathrm{~cm}$. However, the time vision data are available during walking is small since the camera on the front of the right shoe needs to see the LEDs on the back of the left shoe. This makes it difficult to measure movements other than continuous walking along a straight line. Other researchers who used ultrasound for 
position estimation are Huitema et al. [30, and Zhao and Wang [97. Disadvantage of the systems they presented is the need for stationary transmitters or receivers on the floor. Alternatively, the magnetic method of Kortier et al. 34, as described in section 1.2, could be used for estimating relative foot positions. However, it should be noted that magnetic fields could be disturbed by ferromagnetic materials, which are often present in the floor.

If it is required for an application to assess reaching and grasping performance, the system presented in chapters 4 and 5 can also be used on the upper body. If one places ultrasound/IMU combinations on both hands, the position and orientation of the hands with respect to each other can be estimated. Additional IMUs on the arms and trunk can be added to be able to estimate upper body joint angles as well. Using the ultrasound on the hands will improve the upper body kinematic estimation as well, because of the closed loop information.

In chapter 6 we presented a method to assess balance of stroke subjects during walking in a straight line. Using the described setup it is possible to evaluate other daily-life activities, such as sit-stand, transfers, turning around and nonrepetitive walking patterns as well. The quantitative analysis of these variable gait conditions during daily-life activities (without the need for a lab bound system) have hardly been investigated [77. Especially in ambulatory settings, the stability of (stroke) patients during these variable gait phases might be of interest, because of the high incidence of falls during these moments. A recommendation for future work is to investigate these variable walking activities using the set-up described in chapter 6 . The method for step length and step width estimation using the triangles, as used in chapter 5 (Figure 5.5), can be used for this, since this is suitable for variable walking. Also the use of smaller force and torque sensors is recommended, since now the shoes are relatively large and heavy. A Matrix of the six-axis force-torque sensors as presented by Brookhuis et al. 8], with a size of 9x9x1 mm each, would be a possible solution for this. However, 1D force sensors could also be used if only leg loading needs to be investigated. If the sensors are implemented in a sole, the system becomes really easy to use in every shoe, and the influence on the walking pattern will be even smaller. Another alternative for center of mass estimation, without he need for force sensors, could be the use of a single IMU on the pelvis [17].

\subsubsection{Soft-tissue artifacts}

Although it was not a goal of this thesis, soft-tissue artifacts are still a problem in human motion capture systems. If these artifacts occur, the sensor is moving with respect to the body segment (the bone) to which it is attached. These artifacts cause inaccurate estimates of body segment orientations and positions. Therefore, the influence of soft-tissue artifacts on the movement estimates depends on the application and the information that is needed. In our presented gait analysis system they are minimal, because the sensors are attached rigidly 
to the shoes. However, the shoe can move with respect to the foot, and therefore some movement artifacts may still be present. Regarding soft tissue artifacts on the upper leg, we performed a pilot study [72, 85] in which we placed 11 IMUs on the upper leg muscles: rectus femoris, vastus medialis and vastus lateralis. One IMU was positioned on the tendon plate behind the quadriceps (iliotibial tract, as used in Xsens MVN [62]) and used as reference. During walking, relative orientations between IMUs with respect to the reference IMU (all on the upper leg) of up to 28.6 degrees were measured [72]. This pilot showed that the ambulatory measurement of deformation of the upper leg is feasible; however, improvement of the measurement technology is required. The recommendation for future research is to use a sensor system that contains smaller and lighter sensors, to investigate soft-tissue artifacts more accurately. It is hypothesized that once the artifacts can be estimated accurately (during different types of movements), it is possible to eliminate these artifacts.

\subsubsection{Sensor to segment calibration}

Another remaining problem is the sensor-segment calibration. Although the sensor-segment calibration procedure can be performed quickly, a sensor can move with respect to a segment after calibration. This causes errors in calculating the segment orientation. These errors are frequently not noticed (immediately) during the measurements. The inclusion of ultrasound transducers in each IMU can help solve this problem, for example by detecting if a sensor is displaced with respect to the segment.

To conclude this thesis; a click-on-and-play human motion capture system is feasible. A method is presented for the location identification of inertial sensors. This will reduce errors and set-up time of ambulatory systems. Furthermore, a gait analysis system is presented with sensors only on the feet. Not only is this system ambulant and easy to use, it is also shown to be accurate for gait analysis and balance assessment. 


\section{References}

[1] N. Amini, M. Sarrafzadeh, A. Vahdatpour, and W. Xu, "Accelerometerbased on-body sensor localization for health and medical monitoring applications," Pervasive and Mobile Computing, vol. 7, no. 6, pp. 746 - 760, 2011. [Online]. Available: http://www.sciencedirect.com/science/ article/pii/S1574119211001222 (Cited on pages 13 26 and 32 )

[2] A. Avci, S. Bosch, M. Marin-Perianu, R. S. Marin-Perianu, and P. J. M. Havinga, "Activity recognition using inertial sensing for healthcare, wellbeing and sports applications: A survey," in 23th International Conference on Architecture of Computing Systems (ARCS). VDE VERLAG GmbH, February 2010. [Online]. Available: http://ieeexplore.ieee.org/ stamp/stamp.jsp?tp $=$ \&arnumber $=5759000 \&$ isnumber $=5758980$ (Cited on pages $3,18,24$ and 31

[3] C. T. M. Baten, R. H. M. Schoot Uiterkamp, and R. G. V. Wassink, "Can ambulatory analysis of movement be performed sufficiently accurate for decision support in acl surgery rehabilitation," in Gait and Clinical Movement Analysis Society 2012 Annual Conference, 2012, pp. 95-96. [Online]. Available: https://www.amrms.com/ssl/gcmas/2012/ abstract_submission/Baten-1_GCMAS-2012.pdf (Cited on page 14)

[4] K. Berg, "Measuring balance in the elderly: preliminary development of an instrument," Physiotherapy Canada, vol. 41, no. 6, pp. 304-311, 1989. [Online]. Available: http://dx.doi.org/10.3138/ptc.41.6.304 (Cited on page 86

[5] J. H. M. Bergmann and A. H. McGregor, "Body-worn sensor design: What do patients and clinicians want?" Annals of Biomedical Engineering, vol. 39, no. 9, pp. 2299-2312, 2011. [Online]. Available: http://dx.doi.org/10.1007/s10439-011-0339-9 (Cited on pages 85 and 93 .

[6] "Biosyn Systems, Inc. website," 2015, august 17. [Online]. Available: http://www.biosynsystems.net/f-a-b-system/ (Cited on page 7)

[7] J. E. Bortz, "A new mathematical formulation for strapdown inertial navigation," Aerospace and Electronic Systems, IEEE Transactions on, vol. AES-7, no. 1, pp. 61-66, 1971. (Cited on page 69) 
[8] R. A. Brookhuis, H. Droogendijk, M. J. De Boer, R. G. P. Sanders, T. S. J. Lammerink, R. J. Wiegerink, and G. J. M. Krijnen, "Six-axis force-torque sensor with a large range for biomechanical applications," Journal of micromechanics and microengineering, vol. 24, no. 3, p. 035015, 2014. [Online]. Available: http://dx.doi.org/10.1088/0960-1317/24/3/035015 (Cited on page 100)

[9] R. A. Brookhuis, R. G. P. Sanders, K. Ma, T. S. J. Lammerink, M. J. de Boer, G. J. M. Krijnen, and R. J. Wiegerink, "Miniature large range multi-axis force-torque sensor for biomechanical applications," Journal of Micromechanics and Microengineering, vol. 25, no. 2, p. 025012, 2015. [Online]. Available: http://dx.doi.org/10.1088/0960-1317/25/2/025012 (Cited on page 93)

[10] T. Cloete and C. Scheffer, "Benchmarking of a full-body inertial motion capture system for clinical gait analysis," in Engineering in Medicine and Biology Society, 2008. EMBS 2008. 30th Annual International Conference of the IEEE. IEEE, 2008, pp. 4579-4582. [Online]. Available: http: //dx.doi.org/10.1109/IEMBS.2008.4650232 (Cited on pages 13 and 31

[11] A. F. Cordero, H. J. F. M. Koopman, and F. C. T. van der Helm, "Use of pressure insoles to calculate the complete ground reaction forces," Journal of Biomechanics, vol. 37, no. 9, pp. 1427 - 1432, 2004. [Online]. Available: http://dx.doi.org/10.1016/j.jbiomech.2003.12.016 (Cited on page 85)

[12] A. Czernuszenko and A. Czlonkowska, "Risk factors for falls in stroke patients during inpatient rehabilitation," Clinical Rehabilitation, vol. 23, no. 2, pp. 176-188, 2009. [Online]. Available: http: //dx.doi.org/10.1177/0269215508098894 (Cited on page 94)

[13] C. M. Dean and R. B. Shepherd, "Task-related training improves performance of seated reaching tasks after stroke. a randomized controlled trial," Stroke, vol. 28, no. 4, pp. 722-728, 1997. (Cited on page 47)

[14] R. Drillis, R. Contini, and M. Bluestein, "Body segment parameters," Artificial limbs, vol. 8, no. 1, pp. 44-66, 1964. (Cited on page 89)

[15] W. Dubitzky, Data mining techniques in grid computing environments. John Wiley \& Sons, Ltd, 2008. [Online]. Available: http://dx.doi.org/10. 1002/9780470699904 (Cited on page 18)

[16] Y. Ehara, H. Fujimoto, S. Miyazaki, M. Mochimaru, S. Tanaka, and S. Yamamoto, "Comparison of the performance of 3d camera systems ii," Gait $\&$ Posture, vol. 5, no. 3, pp. 251 - 255, 1997. [Online]. Available: http: //www.sciencedirect.com/science/article/pii/S0966636296010934 (Cited on page 55 
[17] M. J. Floor-Westerdijk, H. M. Schepers, P. H. Veltink, E. H. F. van Asseldonk, and J. H. Buurke, "Use of inertial sensors for ambulatory assessment of center-of-mass displacements during walking," Biomedical Engineering, IEEE Transactions on, vol. 59, no. 7, pp. 2080-2084, July 2012. [Online]. Available: http://dx.doi.org/10.1109/TBME.2012.2197211 (Cited on page 100 )

[18] E. Foxlin, "Pedestrian tracking with shoe-mounted inertial sensors," Computer Graphics and Applications, IEEE, vol. 25, no. 6, pp. 38-46, Nov 2005. [Online]. Available: http://dx.doi.org/10.1109/MCG.2005.140 (Cited on page 81)

[19] "Fusion 3d website," 2015, may 26. [Online]. Available: http: //www.fusion3d.org/ (Cited on page 9)

[20] R. K. Ganti, S. Srinivasan, and A. Gacic, "Multisensor fusion in smartphones for lifestyle monitoring," in Body Sensor Networks (BSN), 2010 International Conference on, June 2010, pp. 36-43. [Online]. Available: http://dx.doi.org/10.1109/BSN.2010.10 (Cited on page 5 )

[21] A. A. G. Geessink, B. Verstoep, F. J. van der Hoek, and M. van der Coelen, "Happy feet," B2 Project Electrical Engineering, University of Twente, Enschede, the Netherlands, Tech. Rep., 2012. (Cited on page 6)

[22] F. Gustafsson, Statistical sensor fusion, 1st ed. Studentlitteratur, 2010. (Cited on pages $55,69,70,71$ and 73 )

[23] E. M. Gutierrez-Farewik, A. Bartonek, and H. Saraste, "Comparison and evaluation of two common methods to measure center of mass displacement in three dimensions during gait," Human Movement Science, vol. 25, no. 2, pp. 238 - 256, 2006. [Online]. Available: http://dx.doi.org/10.1016/j.humov.2005.11.001 (Cited on page 85)

[24] L. Hak, H. Houdijk, P. van der Wurff, M. R. Prins, A. Mert, P. J. Beek, and J. H. van Dieën, "Stepping strategies used by post-stroke individuals to maintain margins of stability during walking," Clinical Biomechanics, vol. 28, no. 9, pp. 1041 - 1048, 2013. [Online]. Available: http://dx.doi.org/10.1016/j.clinbiomech.2013.10.010 (Cited on page 93)

[25] M. Hall, E. Frank, G. Holmes, B. Pfahringer, P. Reutemann, and I. H. Witten, "The weka data mining software: an update," ACM SIGKDD Explorations Newsletter, vol. 11, no. 1, pp. 10-18, nov 2009. [Online]. Available: http://dx.doi.org/10.1145/1656274.1656278 (Cited on pages 18 and 34

[26] A. L. Hof, M. G. J. Gazendam, and W. E. Sinke, "The condition for dynamic stability," Journal of Biomechanics, vol. 38, no. 1, pp. 1-8, 2005. [Online]. Available: http://dx.doi.org/10.1016/j.jbiomech.2004.03.025 (Cited on pages 85 and 89 ) 
[27] A. L. Hof, "Scaling gait data to body size," Gait \& Posture, vol. 4, no. 3, pp. 222 - 223, 1996. [Online]. Available: http: //dx.doi.org/10.1016/0966-6362(95)01057-2 (Cited on pages 89 and 91)

[28] W. Hofman, "Instrumentation of rssi measurements for an intelligent wireless motion capturing system using multiple high-end sensors," Bachelor thesis, Saxion Hogescholen, Enschede, the Netherlands, 2011. (Cited on page 6

[29] J. D. Hol, F. Dijkstra, H. J. Luinge, and T. B. Schön, "Tightly coupled uwb/imu pose estimation," in Proceedings of the IEEE International Conference on Ultra-Wideband (ICUWB), Sept 2009, pp. 688-692. [Online]. Available: http://dx.doi.org/10.1109/ICUWB.2009.5288724 (Cited on page 81)

[30] R. B. Huitema, A. L. Hof, and K. Postema, "Ultrasonic motion analysis system - measurement of temporal and spatial gait parameters," Journal of biomechanics, vol. 35, no. 6, pp. 837-842, 2002. [Online]. Available: http://dx.doi.org/10.1016/S0021-9290(02)00032-5 (Cited on pages 4763 81 and 100

[31] T. N. Hung and Y. S. Suh, "Inertial sensor-based two feet motion tracking for gait analysis," Sensors, vol. 13, no. 5, pp. 5614-5629, 2013. [Online]. Available: http://dx.doi.org/10.3390/s130505614 (Cited on pages 6 64 and 99 .

[32] F. Huxham, J. Gong, R. Baker, M. Morris, and R. Iansek, "Defining spatial parameters for non-linear walking," Gait \& P Posture, vol. 23, no. 2, pp. 159 - 163, 2006. [Online]. Available: http: //dx.doi.org/10.1016/j.gaitpost.2005.01.001 (Cited on pages 75 and 91)

[33] H. Jagos, J. Oberzaucher, M. Reichel, W. L. Zagler, and W. Hlauschek, "A multimodal approach for insole motion measurement and analysis," Procedia Engineering, vol. 2, no. 2, pp. 3103 - 3108, 2010, the Engineering of Sport 8 - Engineering Emotion. [Online]. Available: http://dx.doi.org/10.1016/j.proeng.2010.04.118 (Cited on page 7)

[34] H. G. Kortier, J. Antonsson, H. M. Schepers, F. Gustafsson, and P. H. Veltink, "Hand pose estimation by fusion of inertial and magnetic sensing aided by a permanent magnet," Neural Systems and Rehabilitation Engineering, IEEE Transactions on, vol. 23, no. 5, pp. 796-806, September 2015. [Online]. Available: http: //dx.doi.org/10.1109/TNSRE.2014.2357579 (Cited on pages 8 and 100)

[35] J. B. Kuipers, Quaternions and Rotation Sequences: A Primer with Applications to Orbits, Aerospace, and Virtual Reality. Princeton University Press, 2002. (Cited on page 51) 
[36] U. M. Küng, C. G. C. Horlings, F. Honegger, H. P. H. Kremer, B. R. Bloem, B. P. C. van De Warrenburg, and J. H. J. Allum, "Postural instability in cerebellar ataxia: Correlations of knee, arm and trunk movements to center of mass velocity," Neuroscience, vol. 159, no. 1, pp. 390 - 404, 2009. [Online]. Available: http://dx.doi.org/10.1016/j.neuroscience.2008.11.050 (Cited on page 3)

[37] K. Kunze and P. Lukowicz, "Using acceleration signatures from everyday activities for on-body device location," in Wearable Computers, 11th IEEE International Symposium on, Oct 2007, pp. 115-116. [Online]. Available: http://dx.doi.org/10.1109/ISWC.2007.4373794 (Cited on page 13)

[38] K. Kunze, P. Lukowicz, H. Junker, and G. Tröster, "Where am i: Recognizing on-body positions of wearable sensors," in Location- and Context-Awareness, ser. Lecture Notes in Computer Science, T. Strang and C. Linnhoff-Popien, Eds. Springer Berlin Heidelberg, 2005, vol. 3479, pp. 264-275. [Online]. Available: http://dx.doi.org/10.1007/11426646_25 (Cited on pages 13 and 26 )

[39] N. Landwehr, M. Hall, and E. Frank, "Logistic model trees," Machine Learning, vol. 59, no. 1-2, pp. 161-205, 2005. [Online]. Available: http://dx.doi.org/10.1007/s10994-005-0466-3 (Cited on page 34)

[40] S. M. Lasassmeh and J. M. Conrad, "Time synchronization in wireless sensor networks: a survey," in IEEE SoutheastCon 2010 (SoutheastCon), Proceedings of the. IEEE, 2010, pp. 242-245. (Cited on page 59)

[41] C. K. Lim, Z. Luo, I.-M. Chen, and S. H. Yeo, "Wearable wireless sensing system for capturing human arm motion," Sensors and Actuators A: Physical, vol. 166, 2011. [Online]. Available: http: //dx.doi.org/10.1016/j.sna.2010.10.015 (Cited on pages 13 and 31)

[42] T. Liu, Y. Inoue, and K. Shibata, "A wearable ground reaction force sensor system and its application to the measurement of extrinsic gait variability," Sensors, vol. 10, no. 11, pp. 10240-10 255, 2010. [Online]. Available: http://dx.doi.org/10.3390/s101110240 (Cited on pages 63 and 85 )

[43] V. Lugade and K. Kaufman, "Dynamic stability margin using a marker based system and tekscan: A comparison of four gait conditions," Gait Ef Posture, vol. 40, no. 1, pp. 252 - 254, 2014. [Online]. Available: http://dx.doi.org/10.1016/j.gaitpost.2013.12.023 (Cited on pages 85 and 89

[44] V. Lugade, V. Lin, and L.-S. Chou, "Center of mass and base of support interaction during gait," Gait \& Posture, vol. 33, no. 3, pp. 406 - 411, 2011. [Online]. Available: http://dx.doi.org/10.1016/j.gaitpost.2010.12. 013 (Cited on pages 3 and 85 ) 
[45] H. J. Luinge and P. H. Veltink, "Measuring orientation of human body segments using miniature gyroscopes and accelerometers," Medical and Biological Engineering and Computing, vol. 43, no. 2, pp. 273-282, 2005. [Online]. Available: http://dx.doi.org/10.1007/BF02345966 (Cited on pages 65 and 69.

[46] H. J. Luinge, "Inertial sensing of human movement," Ph.D. dissertation, University of Twente, Enschede, the Netherlands, October 2002. [Online]. Available: http://doc.utwente.nl/38637/ (Cited on page 6)

[47] A. Mannini, A. M. Sabatini, and S. S. Intille, "Accelerometry-based recognition of the placement sites of a wearable sensor," Pervasive and Mobile Computing, vol. 21, pp. 62 - 74, 2015. [Online]. Available: http://dx.doi.org/10.1016/j.pmcj.2015.06.003 (Cited on page 99)

[48] J. Mao, "Optimal orthonormalization of the strapdown matrix by using singular value decomposition," Computers $\&$ mathematics with applications, vol. 12, no. 3, Part 1, pp. 353-362, 1986. [Online]. Available: http://dx.doi.org/10.1016/0898-1221(86)90194-X (Cited on page 73 )

[49] A. Martínez-Ramírez, D. Weenk, P. Lecumberri, N. Verdonschot, D. Pakvis, and P. H. Veltink, "Pre-operative ambulatory measurement of asymmetric lower limb loading during walking in total hip arthroplasty patients," Journal of NeuroEngineering and Rehabilitation, vol. 10, no. 1, pp. 1-10, 2013. [Online]. Available: http://dx.doi.org/10. 1186/1743-0003-10-41 (Cited on page 3)

[50] A. Martínez-Ramírez, D. Weenk, P. Lecumberri, N. Verdonschot, D. Pakvis, and P. H. Veltink, "Assessment of asymmetric leg loading before and after total hip arthroplasty using instrumented shoes," Journal of NeuroEngineering and Rehabilitation, vol. 11, no. 1, p. 20, 2014. [Online]. Available: http://dx.doi.org/10.1186/1743-0003-11-20 (Cited on page 3

[51] A. Martínez-Ramírez, D. Weenk, P. Lecumberri, N. Verdonschot, D. Pakvis, and P. H. Veltink, "Preoperative ambulatory measurement of asymmetric leg loading during sit-to-stand in hip arthroplasty patients," Neural Systems and Rehabilitation Engineering, IEEE Transactions on, vol. 22, no. 3, pp. 585-592, May 2014. [Online]. Available: http: //dx.doi.org/10.1109/TNSRE.2013.2263394 (Cited on page 3)

[52] H. B. Menz, M. D. Latt, A. Tiedemann, M. M. S. Kwan, and S. R. Lord, "Reliability of the gaitriteß walkway system for the quantification of temporo-spatial parameters of gait in young and older people," Gait E Posture, vol. 20, no. 1, pp. 20 - 25, 2004. [Online]. Available: http://dx.doi.org/10.1016/S0966-6362(03)00068-7 (Cited on page 85) 
[53] H. op den Akker, "Smart tailoring of real-time physical activity coaching systems," Ph.D. dissertation, University of Twente, Enschede, October 2014. [Online]. Available: http://dx.doi.org/10.3990/1.9789036537629 (Cited on page 3 )

[54] J. Perry, M. Garrett, J. K. Gronley, and S. J. Mulroy, "Classification of walking handicap in the stroke population," Stroke, vol. 26, no. 6, pp. 982-989, 1995. [Online]. Available: http://dx.doi.org/10.1161/01.STR.26. 6.982 (Cited on page 85 )

[55] C. L. A. Pollock, J. J. B. Eng, and S. J. C. Garland, "Clinical measurement of walking balance in people post stroke: A systematic review," Clinical Rehabilitation, vol. 25, no. 8, pp. 693-708, 2011. [Online]. Available: http://dx.doi.org/10.1177/0269215510397394 (Cited on page 85

[56] J. R. Rebula, L. V. Ojeda, P. G. Adamczyk, and A. D. Kuo, "Measurement of foot placement and its variability with inertial sensors," Gait 8 Posture, vol. 38, no. 4, pp. 974 - 980, 2013. [Online]. Available: http://dx.doi.org/10.1016/j.gaitpost.2013.05.012 (Cited on pages 63 and 85 )

[57] P. P. L. Regtien, F. Van Der Heijden, M. J. Korsten, and W. Olthuis, Measurement science for engineers. Elsevier, 2004. [Online]. Available: http://www.sciencedirect.com/science/book/9781903996584 (Cited on pages $48,59,66$ and 81 )

[58] D. Roetenberg, "Inertial and magnetic sensing of human motion," Ph.D. dissertation, University of Twente, 2006. (Cited on pages 6 and 8)

[59] D. Roetenberg, P. Slycke, A. Ventevogel, and P. H. Veltink, "A portable magnetic position and orientation tracker," Sensors and Actuators A: Physical, vol. 135, no. 2, pp. 426 - 432, 2007. [Online]. Available: http: //www.sciencedirect.com/science/article/pii/S092442470600553X (Cited on pages 847 and 63 .

[60] D. Roetenberg, P. J. Slycke, and P. H. Veltink, "Ambulatory position and orientation tracking fusing magnetic and inertial sensing," Biomedical Engineering, IEEE Transactions on, vol. 54, no. 5, pp. 883-890, 2007. [Online]. Available: http://dx.doi.org/10.1109/TBME.2006.889184 (Cited on page 13

[61] D. Roetenberg, H. J. Luinge, C. T. M. Baten, and P. H. Veltink, "Compensation of magnetic disturbances improves inertial and magnetic sensing of human body segment orientation," Neural Systems and Rehabilitation Engineering, IEEE Transactions on, vol. 13, no. 3, pp. 395-405, 2005. [Online]. Available: http://dx.doi.org/10.1109/TNSRE. 2005.847353 (Cited on pages 65 and 67) 
[62] D. Roetenberg, H. J. Luinge, and P. Slycke, "Xsens mvn: full 6dof human motion tracking using miniature inertial sensors," Xsens Motion Technologies BV, Tech. Rep, 2009. (Cited on pages 5, 7, 8, 31, 47, 63, 81 and 101)

[63] H. Rouhani, J. Favre, X. Crevoisier, and K. Aminian, "Ambulatory assessment of $3 \mathrm{~d}$ ground reaction force using plantar pressure distribution," Gait $\&$ Posture, vol. 32, no. 3, pp. 311 - 316, 2010. [Online]. Available: http://dx.doi.org/10.1016/j.gaitpost.2010.05.014 (Cited on pages 63 and 85

[64] A. Sabatini, C. Martelloni, S. Scapellato, and F. Cavallo, "Assessment of walking features from foot inertial sensing," Biomedical Engineering, IEEE Transactions on, vol. 52, no. 3, pp. 486-494, March 2005. [Online]. Available: http://dx.doi.org/10.1109/TBME.2004.840727 (Cited on page 85

[65] C. M. Said, P. A. Goldie, A. E. Patla, E. Culham, W. A. Sparrow, and M. E. Morris, "Balance during obstacle crossing following stroke," Gait ES Posture, vol. 27, no. 1, pp. 23 - 30, 2008. [Online]. Available: http: //www.sciencedirect.com/science/article/pii/S0966636207000069 (Cited on page 47)

[66] H. M. Schepers, "Ambulatory assessment of human body kinematics and kinetics," Ph.D. dissertation, University of Twente, Enschede, the Netherlands, June 2009. [Online]. Available: http://dx.doi.org/10.3990/ 1.9789036528443 (Cited on pages 6 6 15,16 and 58

[67] H. M. Schepers, H. F. J. M. Koopman, and P. H. Veltink, "Ambulatory assessment of ankle and foot dynamics," Biomedical Engineering, IEEE Transactions on, vol. 54, no. 5, pp. 895-902, 2007. [Online]. Available: http://dx.doi.org/10.1109/TBME.2006.889769 (Cited on pages 63 85 and 86 )

[68] H. M. Schepers, D. Roetenberg, and P. H. Veltink, "Ambulatory human motion tracking by fusion of inertial and magnetic sensing with adaptive actuation," Medical \& Biological Engineering $\&$ Computing, vol. 48, no. 1, pp. 27-37, 2010. [Online]. Available: http://dx.doi.org/10.1007/ s11517-009-0562-9 (Cited on pages 7 and 69)

[69] H. M. Schepers, E. van Asseldonk, J. H. Buurke, and P. H. Veltink, "Ambulatory estimation of center of mass displacement during walking," Biomedical Engineering, IEEE Transactions on, vol. 56, no. 4, pp. 1189-1195, 2009. [Online]. Available: http://dx.doi.org/10.1109/TBME. 2008.2011059 (Cited on pages 6, 763 and 89)

[70] H. M. Schepers, E. H. Van Asseldonk, C. Baten, and P. H. Veltink, "Ambulatory estimation of foot placement during walking using inertial sensors," Journal of biomechanics, vol. 43, no. 16, pp. 3138-3143, 2010. 
[Online]. Available: http://dx.doi.org/10.1016/j.jbiomech.2010.07.039 (Cited on pages 47 and 63 )

[71] I. Skog, P. Handel, J.-O. Nilsson, and J. Rantakokko, "Zero-velocity detection - an algorithm evaluation," Biomedical Engineering, IEEE Transactions on, vol. 57, no. 11, pp. 2657-2666, 2010. [Online]. Available: http://dx.doi.org/10.1109/TBME.2010.2060723 (Cited on page 71)

[72] A. Stevens, "Measuring deformation of the m. quadriceps femoris with inertial and magnetic sensors," Bachelor thesis, University of Twente, Enschede, the Netherlands, 2012. (Cited on page 101)

[73] W. Tao, T. Liu, R. Zheng, and H. Feng, "Gait analysis using wearable sensors," Sensors, vol. 12, no. 2, pp. 2255-2283, 2012. [Online]. Available: http://dx.doi.org/10.3390/s120202255 (Cited on pages 3 4.6 and 63)

[74] D. H. Titterton and J. L. Weston, Strapdown inertial navigation technology, 2nd ed. The Institution of Electrical Engineers, 2004. (Cited on page 6)

[75] J. van den Noort, M. van der Esch, M. P. Steultjens, J. Dekker, M. Schepers, P. H. Veltink, and J. Harlaar, "Influence of the instrumented force shoe on gait pattern in patients with osteoarthritis of the knee," Medical \& Biological Engineering \& Computing, vol. 49, no. 12, pp. 1381-1392, 2011. [Online]. Available: http://dx.doi.org/10.1007/ s11517-011-0818-z (Cited on pages 85 and 93 )

[76] F. Van Der Heijden, R. P. W. Duin, D. De Ridder, and D. M. J. Tax, Classification, parameter estimation and state estimation: an engineering approach using MATLAB. Wiley, 2004. (Cited on page 73)

[77] F. B. van Meulen, J. Reenalda, J. H. Buurke, and P. H. Veltink, "Assessment of daily-life reaching performance after stroke," Annals of Biomedical Engineering, vol. 43, no. 2, pp. 478-486, 2015. [Online]. Available: http://dx.doi.org/10.1007/s10439-014-1198-y (Cited on pages 5 $31 \quad 33 \quad 85,86$ and 100

[78] C. L. Vaughan, B. L. Davis, and J. C. O'connor, Dynamics of human gait. Human Kinetics Publishers USA, 1992. (Cited on page 58)

[79] P. H. Veltink, F. v. d. Helm, B. Koopman, and A. Nene, Dictaat Biomechatronics. University of Twente, 2008. (Cited on page 8)

[80] D. Vlasic, R. Adelsberger, G. Vannucci, J. Barnwell, M. Gross, W. Matusik, and J. Popović, "Practical motion capture in everyday surroundings," ACM Trans. Graph., vol. 26, no. 3, Jul. 2007. [Online]. Available: http://dx.doi.org/10.1145/1276377.1276421 (Cited on pages 6 and 63. 
[81] R. G. V. Wassink, C. T. M. Baten, P. H. Veltink, R. N. J. Veldhuis, and J. H. Smeding, "Monitoring of human activities using a trainable system based on Hidden Markov modelling technology," Gait and Posture, vol. 24, no. Supplement 2, pp. S109-S110, 2006. [Online]. Available: http://dx.doi.org/10.1016/j.gaitpost.2006.11.076 (Cited on pages 324 and 31 )

[82] D. Weenk, "Center of mass estimation in mvn," Internship, Xsens Technologies B. V., Enschede, the Netherlands, Tech. Rep., 2010. (Cited on page 7 (7)

[83] D. Weenk, "Automatic identification of inertial sensors on the human body segments," Master's thesis, University of Twente, 2011. (Cited on page 7)

[84] D. Weenk, D. Roetenberg, B.-J. F. van Beijnum, H. J. Hermens, and P. H. Veltink, "Ambulatory estimation of relative foot positions by fusing ultrasound and inertial sensor data," Neural Systems and Rehabilitation Engineering, IEEE Transactions on, vol. 23, no. 5, pp. 817-826, September 2015. [Online]. Available: http://dx.doi.org/10.1109/ TNSRE.2014.2357686 (Cited on pages 85 and 88 )

[85] D. Weenk, A. G. Stevens, B. H. W. Koning, B. J. F. van Beijnum, H. J. Hermens, and P. H. Veltink, "A feasibility study in measuring soft tissue artifacts on the upper leg using inertial and magnetic sensors," in Fourth Dutch Conference on Bio-Medical Engineering, Egmond aan Zee, The Netherlands. Egmond aan Zee, The Netherlands: BME, January 2013, pp. 154-154. (Cited on page 101)

[86] D. Weenk, B. J. F. van Beijnum, A. Droog, H. J. Hermens, and P. H. Veltink, "Ultrasonic range measurements on the human body," in Seventh International Conference on Sensing Technology, ICST 2013, Wellington, New Zealand. IEEE, December 2013, pp. 151-156. [Online]. Available: http://dx.doi.org/10.1109/ICSensT.2013.6727633 (Cited on pages 66, 67 74 75 . 77 and 88 .

[87] D. Weenk, F. B. van Meulen, B.-J. F. van Beijnum, and P. H. Veltink, "Ambulatory gait analysis in stroke patients using ultrasound and inertial sensors," in 13th International Symposium on 3D Analysis of Human Movement, 3D-AHM 2014, Lausanne, Switserland. EPFL, July 2014, pp. 385-388. [Online]. Available: http://doc.utwente.nl/91437/ (Cited on page 93)

[88] D. Weenk, B.-J. F. van Beijnum, C. T. M. Baten, H. J. Hermens, and P. H. Veltink, "Automatic identification of inertial sensor placement on human body segments during walking," Journal of NeuroEngineering and Rehabilitation, vol. 10, no. 1, p. 31, 2013. [Online]. Available: http://dx.doi.org/10.1186/1743-0003-10-31 (Cited on pages 31, 33, 36, 42 , and 43 ) 
[89] "Weka website," 2012, july 30. [Online]. Available: http://www.cs. waikato.ac.nz/ml/weka/ (Cited on pages 18 and 34 )

[90] G. Welch and G. Bishop, "An introduction to the kalman filter," Technical Report, University of North Carolina at Chapel Hill, Tech. Rep., 2006. (Cited on pages 70 and 71 )

[91] I. H. Witten and E. Frank, Data Mining: Practical machine learning tools and techniques, 2nd ed. Morgan Kaufmann Publishers, 2005. [Online]. Available: http://store.elsevier.com/product.jsp?isbn $=9780080477022$ (Cited on pages 18,26 and 34 )

[92] Xsens MVN User Manual, Xsens Technologies B. V., May 2010. (Cited on pages 142532 and 43 .

[93] "Xsens Technologies B. V. website," 2015, march 13. [Online]. Available:

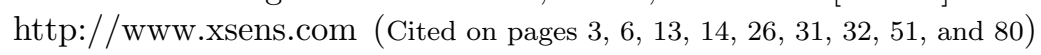

[94] W. Xu, M. Zhang, A. A. Sawchuk, and M. Sarrafzadeh, "Robust human activity and sensor location corecognition via sparse signal representation," Biomedical Engineering, IEEE Transactions on, vol. 59, no. 11, pp. 3169-3176, Nov 2012. [Online]. Available: http: //dx.doi.org/10.1109/TBME.2012.2211355 (Cited on pages 31 and 99 )

[95] Z. Yu and G. C. M. Meijer, "A programmable time-gain-compensation (TGC) amplifier for medical ultrasonic echo signal processing," in SolidState and Integrated-Circuit Technology, 2008. ICSICT 2008. 9th International Conference on. IEEE, 2008, pp. 1725-1728. (Cited on page 59)

[96] N. Zaurino, "Sensing height differences on human body using barometers," Master's thesis, University of Pisa, 2012. (Cited on page 6)

[97] H. Zhao and Z. Wang, "Motion measurement using inertial sensors, ultrasonic sensors, and magnetometers with extended kalman filter for data fusion," Sensors Journal, IEEE, vol. 12, no. 5, pp. 943-953, 2012. [Online]. Available: http://dx.doi.org/10.1109/JSEN.2011.2166066 (Cited on pages 63 and 100

[98] H. Zhou, T. Stone, H. Hu, and N. Harris, "Use of multiple wearable inertial sensors in upper limb motion tracking," Medical engineering \&5 physics, vol. 30, no. 1, pp. 123-133, 2008. [Online]. Available: http://dx.doi.org/10.1016/j.medengphy.2006.11.010 (Cited on pages 13 and 31 


\section{Dankwoord}

Allereerst wil ik graag mijn co-promotor dr. ir. Bert-Jan van Beijnum, en mijn promotoren prof. dr. ir. Peter Veltink en prof. dr. ir. Hermie Hermens bedanken voor het aanbieden van de afstudeer- en promotieplaats in het FUSION project. Bert-Jan, sinds mijn afstuderen ben je al mijn dagelijkse begeleider. Daarna hadden we vier jaar lang, bijna wekelijks, een overleg over de voortgang van het onderzoek en ook hierna hebben we nog erg prettig samengewerkt tijdens het ARMOR project. Hiervoor wil ik je hartelijk bedanken! Peter, tijdens mijn stage bij Xsens, begin 2010, belde je me op met de vraag of ik interesse had in een promotieplek bij BSS in het FUSION project. Ik kon dan al direct tijdens mijn afstuderen hieraan werken en zo met een vliegende start beginnen aan mijn promotieonderzoek. Ik wil je bedanken voor de begeleiding en de discussies tijdens de voortgangsbesprekingen waaruit iedere keer weer nieuwe ideeën ontstonden. Hermie, ook jij heel erg bedankt voor de begeleiding, je input tijdens de discussies en de feedback op de teksten voor de congressen en de tijdschriften.

Daniel Roetenberg, tijdens mijn stage bij Xsens was je al een erg goede begeleider, en wist je me te motiveren voor dit onderzoek. Ik heb erg veel van je geleerd tijdens mijn onderzoek en met name tijdens het werken aan hoofdstuk 5 bedankt hiervoor! Ook Henk Luinge en Martin Schepers en de rest van de mensen bij Xsens wil ik bedanken voor de begeleiding en de leuke tijd tijdens mijn stage, afstuderen en promotieonderzoek.

Chris Baten, bedankt voor het coördineren van het FUSION project en de begeleiding tijdens mijn afstuderen en het onderzoek daarna. Bart Koning, ook jou wil ik bedanken voor de goede samenwerking. De metingen die we samen deden verliepen altijd erg prettig. Ook wil ik graag de andere medewerkers van Roessingh Research and Development bedanken voor de hulp tijdens de metingen. De overige FUSION projectpartners wil ik hartelijk danken voor de samenwerking. En natuurlijk alle proefpersonen, zonder jullie was dit onderzoek niet mogelijk geweest. Bedankt voor het dragen van alle verschillende sensoren.

Verder wil ik graag Wesley, Nicola, Alien, Arno, Bart, Frank, Michiel, Demian, Steven, Jermain, Emil, Paul en Salma bedanken. Niet alleen was het erg prettig om jullie te mogen begeleiden tijdens jullie afstudeer- of stageopdracht, ook was het werk dat jullie hebben gedaan nodig voor de totstand- 
koming van dit proefschrift.

Wies, bedankt dat je altijd klaar stond om te helpen als er weer eens een afspraak gepland moest worden of als er andere zaken geregeld dienden te worden. Leuk dat je nu mijn nieuwe buurvrouw bent. Sandra, ook jou wil ik bedanken voor de ondersteuning tijdens het afronden van dit proefschrift.

Ed, Marcel en Victor, bedankt voor jullie ondersteuning en het meedenken bij veel technische zaken; van het ontwerpen en solderen van het ultrasoundsysteem tot het zorgen dat ik een computer had om metingen mee te kunnen doen.

Vanaf februari dit jaar mocht ik werken aan het ARMOR project. Veel van de algoritmes, beschreven in dit proefschrift, hebben we in dit project (met hier en daar wat aanpassingen) direct kunnen toepassen in de praktijk. Dit was erg leuk om te doen en ik wil dan ook graag Bertil, Henk, Roos en BertJan bedanken voor deze mogelijkheid en de prettige samenwerking. Succes met de afronding van dit leuke project!

Fokke, Frauke, Frederiek, Henk, Hossein, Kees, Robert-Jan, Thijs en Rick, bedankt voor de leuke tijd tijdens de whisky tasting in Edinburgh. Hopelijk gaan we binnenkort nog een keer zo'n uitje organiseren en wellicht bestellen we samen nog eens een dozijn. Ook wil ik alle andere collega's bij de vakgroep Biomedische Signalen en Systemen bedanken voor de prettige samenwerking, de input tijdens de maandelijkse group meetings en ook voor de gezellige momenten in de coffeecorner, tijdens de vakgroepuitjes en natuurlijk onze tweejaarlijkse reizen naar Egmond aan Zee.

Fokke en Henk, we hebben ongeveer vier jaar met elkaar op een kamer gezeten. De samenwerking verliep erg prettig, de sfeer was goed en hopelijk hebben onze opvolgers ook nog plezier van het soundboard en de koffie- en voerkostenlijsten. Bedankt dat jullie mij bij willen staan tijdens de verdediging.

Vrienden en familie, ik ga jullie niet allemaal bij naam noemen, maar ik wil jullie allemaal bedanken voor jullie interesse en natuurlijk voor de gezellige momenten buiten het onderzoek om. Guus, bedankt voor de geweldige muziek. $\mathrm{Pa}$ en ma, bedankt voor jullie steun en interesse, al zullen jullie vaak geen idee hebben gehad waar ik het over had. Aniek, jij hebt vooral het laatste jaar nog leuker gemaakt. Bedankt voor je steun en begrip als ik weer eens tot 's avonds laat nog aan het werk moest. 


\section{Biography}

Dirk Weenk was born in Arnhem, The Netherlands, in 1984. He finished his VWO at the Rietveld Lyceum in Doetinchem in 2003. After his VWO he started studying electrical engineering at the University of Twente, Enschede, The Netherlands. In 2010 he did a four-month internship at Xsens Technologies B. V. about center of mass estimation in MVN. In May 2010 he started working in the Biomedical Signals and Signals group of prof. dr. ir. P. H. Veltink on the FUSION project. First towards his MSc - which he received in 2011 after which he continued working on the project towards his PhD. In February 2015 he started working on the ARMOR project on Ambulant Registration of Military Operational Readiness, together with Evalan

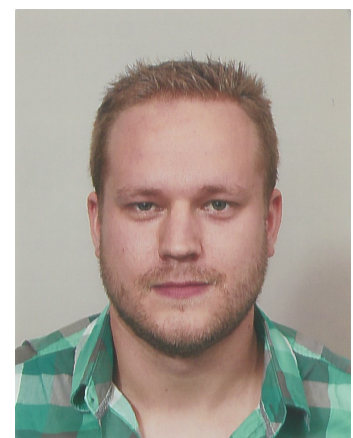
B. V. and The Netherlands Ministry of Defence. 


\section{List of Publications}

\section{Journal papers}

\section{5}

Submitted: Weenk, D. and van Beijnum, B. J. F. and Baten, C. T. M. and Hermens, H. J. and Veltink, P. H. (2015) "On-Body Inertial Sensor Location and Activity Recognition". chapter 3

In preparation: van Meulen, F. B. and Weenk, D and Buurke, J. H. and van Beijnum, B. J. F. and Veltink, P. H. (2015) "Ambulatory Assessment of Walking Balance after Stroke". chapter 6

Weenk, D. and Roetenberg, D. and van Beijnum, B. J. F. and Hermens, H. J. and Veltink, P. H. (2015) "Ambulatory Estimation of Relative Foot Positions by Fusing Ultrasound and Inertial Sensor Data", IEEE transactions on neural systems and rehabilitation engineering, vol.23, no.5, pp.817-826, Sept. 2015 chapter 5

\section{4}

Martínez-Ramírez, A. and Weenk, D. and Lecumberri, P. and Verdonschot, N. and Pakvis, D. and Veltink, P. H. (2014) "Pre-operative Ambulatory Measurement of Asymmetric Leg Loading during Sit to Stand in Hip Arthroplasty Patients", IEEE transactions on neural systems and rehabilitation engineering, 22 (3). pp. 585-592. ISSN 1534-4320

\section{3}

Martínez-Ramírez, A. and Weenk, D. and Lecumberri, P. and Verdonschot, N. and Pakvis, D. and Veltink, P. H. (2013) "Pre-operative Ambulatory Measurement of Asymmetric Lower Limb Loading during Walking in Total Hip Arthroplasty Patients", Journal of NeuroEngineering and Rehabilitation, 10 (41). pp. 1-10. ISSN 1743-0003 
Martínez-Ramírez, A. and Weenk, D. and Lecumberri, P. and Verdonschot, N. and Pakvis, D. and Veltink, P. H. (2013) "Assessment of Asymmetric Leg Loading Before and After Total Hip Arthroplasty using Instrumented Shoes", Journal of NeuroEngineering and Rehabilitation, 11 (20). pp. 1-9. ISSN 17430003

Weenk, D. and van Beijnum, B. J. F. and Baten, C. T. M. and Hermens, H. J. and Veltink, P. H. (2013) "Automatic Identification of Inertial Sensor Placement on Human Body Segments during Walking", Journal of NeuroEngineering and Rehabilitation, 10 (31). pp. 1-9. ISSN 1743-0003 chapter 2

\section{Conference papers}

\section{3}

Weenk, D. and van Beijnum, B. J. F. and Droog, A. and Hermens, H. J. and Veltink, P. H. (2013) "Ultrasonic Range Measurements on the Human Body". In: Seventh International Conference on Sensing Technology, ICST 2013, 3-5 Dec 2013, Wellington, New Zealand. pp. 151-156. IEEE. ISSN 2156-8065 ISBN 978-1-4673-5220-8 chapter 4

\section{Conference abstracts}

\section{5}

Weenk, D. and van Meulen, F. B. and van Beijnum, B. J. F. and Droog, A. and Roetenberg, D. and Hermens, H. J. and Veltink, P. H. (2015) "Gait Analysis Using Ultrasound and Inertial Sensors". In: Book of Abstracts \& Programme of BME 2015: the 5th Dutch Conference on Bio-Medical Engineering, 22-23 Jan 2015, Egmond aan Zee, The Netherlands. BME. ISBN not assigned

Weenk, D. and van Beijnum, B. J. F. and Goaied, S. and Baten, C. T. M. and Hermens, H. J. and Veltink, P. H. (2015) "On-Body Inertial Sensor Location Recognition". In: Book of Abstracts \& Programme of BME 2015: the 5th Dutch Conference on Bio-Medical Engineering, 22-23 Jan 2015, Egmond aan Zee, The Netherlands. BME. ISBN not assigned

\section{4}

Weenk, D. and van Meulen, F. B. and van Beijnum, B. J. F. and Veltink, P. H. (2014) "Ambulatory Gait Analysis in Stroke Patients using Ultrasound and Inertial Sensors". In: 13th International Symposium on 3D Analysis of Human Movement, 3D-AHM 2014, 14-17 July 2014, Lausanne, Switserland. pp. 385-388. EPFL. ISBN 9782880748562 


\section{3}

Weenk, D. and van der Coelen, M. and Geessink, A. A. G. and van der Hoek, F. J. and Verstoep, B. and Kortier, H. G. and van Meulen, F. B. and van Beijnum, B. J. F. and Veltink, P. H. (2013) "Ambulatory Estimation of Relative Foot Positions using Ultrasound". In: Fourth Dutch Conference on Bio-Medical Engineering, 24-25 Jan 2013, Egmond aan Zee, The Netherlands. pp. 155-155. BME. ISBN not assigned

Weenk, D. and Stevens, A. G. and Koning, B. H. W. and van Beijnum, B. J. F. and Hermens, H. J. and Veltink, P. H. (2013) "A Feasibility Study in Measuring Soft Tissue Artifacts on the Upper Leg Using Inertial and Magnetic Sensors". In: Fourth Dutch Conference on Bio-Medical Engineering, 24-25 Jan 2013, Egmond aan Zee, The Netherlands. pp. 154-154. BME. ISBN not assigned

\section{1}

Weenk, D. and van Beijnum, B. J. F. and Veltink, P. H. (2011) "Automatic Identification of Inertial Sensors on the Human Body Segments". In: Book of Abstracts of ICAMPAM 2011: the 2nd International Conference on Ambulatory Monitoring of Physical Activity and Movement, 24-27 May 2011, Glasgow, United Kingdom. ICAMPAM. ISBN not assigned

Weenk, D. and van Beijnum, B. J. F. and Veltink, P. H. (2011) "Automatic Identification and Localization of Inertial Sensors on the Human Body". In: Book of Abstracts \& Programme of BME 2011: the 3rd Dutch Conference on Bio-Medical Engineering, 20-21 Jan 2011, Egmond aan Zee, The Netherlands. BME. ISBN not assigned 
ISBN 978-90-365-3972-2

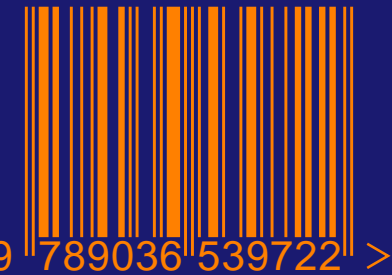

